\title{
Synthesis of Substituted Pyridine Derivatives via the Ruthenium-Catalyzed Cycloisomerization of 3-Azadienynes
}

\author{
Mohammad Movassaghi* and Matthew D. Hill \\ Massachusetts Institute of Technology, Department of Chemistry, Massachusetts 02139
}

\section{Supporting Information}

General Procedures. All reactions were performed in oven-dried or flame-dried round bottomed flasks, modified Schlenk (Kjeldahl shape) flasks, or glass pressure vessels. The flasks were fitted with rubber septa and reactions were conducted under a positive pressure of argon. Stainless steel syringes or cannulae were used to transfer air- and moisture-sensitive liquids. Flash column chromatography was performed as described by Still et al. using silica gel $(60-\AA \AA$ pore size, $32-63 \mu \mathrm{m}$, standard grade, Sorbent Technologies) or non-activated alumina gel (80-325 mesh, chromatographic grade, EM Science). ${ }^{1}$ Analytical thin-layer chromatography was performed using glass plates precoated with $0.25 \mathrm{~mm} \mathrm{230-400} \mathrm{mesh} \mathrm{silica} \mathrm{gel} \mathrm{or} \mathrm{neutral} \mathrm{alumina} \mathrm{gel} \mathrm{impregnated} \mathrm{with} \mathrm{a} \mathrm{fluorescent}$ indicator $(254 \mathrm{~nm})$. Thin layer chromatography plates were visualized by exposure to ultraviolet light and/or by exposure to an ethanolic phosphomolybdic acid (PMA), an acidic solution of $p$ anisaldehyde (anis), an aqueous solution of ceric ammonium molybdate (CAM), an aqueous solution of potassium permanganate $\left(\mathrm{KMnO}_{4}\right)$ or an ethanolic solution of ninhydrin followed by heating $(<1$ min) on a hot plate $\left(\sim 250{ }^{\circ} \mathrm{C}\right)$. Organic solutions were concentrated on Büchi R-200 rotary evaporators at $\sim 10$ Torr (house vacuum) at $25-35{ }^{\circ} \mathrm{C}$, then at $\sim 0.5$ Torr (vacuum pump) unless otherwise indicated.

Materials. Commercial reagents and solvents were used as received with the following exceptions: Dichloromethane, diethyl ether, tetrahydrofuran, acetonitrile, and toluene were purchased from J.T. Baker $\left(\right.$ Cycletainer $^{\mathrm{TM}}$ ) and were purified by the method of Grubbs et al. under positive argon pressure. $^{2}$ Ammonium hexafluorophosphate was dried at $150{ }^{\circ} \mathrm{C}$ under vacuum $(\sim 0.5$ torr $)$ for $24 \mathrm{~h}$ and stored in a glove box under an atmosphere of dinitrogen. The molarity of $n$-butyllithium solutions was determined by titration using diphenylacetic acid as an indicator (average of three determinations). ${ }^{3}$ Hünig's base and 2-chloropyridine were distilled from calcium hydride and stored sealed under an argon atmosphere. The starting amides were prepared by acylation of the corresponding anilines ${ }^{4}$ or via previously reported copper-catalyzed $\mathrm{C}-\mathrm{N}$ bond-forming reactions. ${ }^{5,6}$ The ruthenium complex $5\left(\mathrm{CpRu}\left(\mathrm{PPh}_{3}\right)_{2} \mathrm{Cl}\right)$ is commercially available and was prepared on large scale

\footnotetext{
${ }^{1}$ Still, W. C.; Kahn, M.; Mitra, A. J. Org. Chem. 1978, 43, 2923-2925.

${ }^{2}$ Pangborn, A. B.; Giardello, M. A.; Grubbs, R. H.; Rosen, R. K.; Timmers, F. J. Organometallics 1996, 15, 1518-1520.

${ }^{3}$ Kofron, W. G.; Baclawski, L. M. J. Org. Chem. 1976, 41, 1879-1880.

${ }^{4}$ For a general procedure, see: DeRuiter, J.; Swearingen, B. E,; Wandrekar, V.; Mayfield, C. A. J. Med. Chem. 1989, 32, $1033-1038$.

${ }^{5}$ For the general procedure used for the synthesis of all $N$-vinyl amides, see: Jiang, L.; Job, G. E.; Klapars, A.; Buchwald, S. L. Org. Lett. 2003, 5, 3667-3669.

${ }^{6}$ For related reports, see: (a) Wolfe, J. P.; Wagaw, S.; Marcoux, J.-F.; Buchwald, S. L. Acc. Chem. Res. 1998, 31, 805-818. (b) Hartwig, J. F. Acc. Chem. Res. 1998, 31, 852-860. (c) Hartwig, J. F. Angew. Chem., Int. Ed. 1998, 37, 2046-2067. (d) Yang, B. H.; Buchwald, S. L. J. Organomet. Chem. 1999, 576, 125-146. (e) Muci, A. R.; Buchwald, S. L. Top. Curr. Chem. 2002, 219, 131-209. (f) Beletskaya, I. P.; Cheprakov, A. V. Coordin. Chem. Rev. 2004, 248, 2337-2364. (g) Dehli, J. R.; Legros, J.; Bolm, C. Chem. Commun. 2005, 973-986.
} 
according to a literature procedure. ${ }^{7}$ 2-Dicyclohexyl-phosphino-2', $6^{\prime}$-dimethoxy-1,1'-biphenyl $(\mathrm{SPhos})^{8}$ is commercially available and we thank the Buchwald group for providing samples for initial studies. Solutions of Copper (I) (trimethylysilyl)-acetylide were prepared immediately prior to use according to literature procedure. ${ }^{9}$

Instrumentation. Proton nuclear magnetic resonance $\left({ }^{1} \mathrm{H}\right.$ NMR) spectra were recorded with a Varian inverse probe 500 INOVA spectrometer. Chemical shifts are recorded in parts per million from internal tetramethylsilane on the $\delta$ scale and are referenced from the residual protium in the NMR solvent $\left(\mathrm{CHCl}_{3}: \delta 7.27, \mathrm{C}_{6} \mathrm{HD}_{5}: \delta 7.16\right)$. Data is reported as follows: chemical shift [multiplicity $(\mathrm{s}=$ singlet, $\mathrm{d}=$ doublet, $\mathrm{q}=$ quartet, $\mathrm{m}=$ multiplet), coupling constant(s) in Hertz, integration, assignment]. Carbon-13 nuclear magnetic resonance spectra were recorded with a Varian 500 INOVA spectrometer and are recorded in parts per million from internal tetramethylsilane on the $\delta$ scale and are referenced from the carbon resonances of the solvent $\left(\mathrm{CDCl}_{3}: \delta 77.2\right.$, benzene- $\left.d_{6}: \delta 128.0\right)$. Data is reported as follows: chemical shift [multiplicity ( $\mathrm{s}=$ singlet, $\mathrm{d}=$ doublet, $\mathrm{q}=$ quartet, $\mathrm{m}=$ multiplet), coupling constant(s) in Hertz, assignment]. Infrared data were obtained with a Perkin-Elmer 2000 FTIR and are reported as follows: [frequency of absorption $\left(\mathrm{cm}^{-1}\right)$, intensity of absorption $(\mathrm{s}=\mathrm{strong}, \mathrm{m}=$ medium, $\mathrm{w}=$ weak, $\mathrm{br}=$ broad), assignment]. Combustion analysis was performed by Atlantic Microlab, Incorporated. We are grateful to Dr. Li Li for obtaining the mass spectroscopic data at the Department of Chemistry’s Instrumentation Facility, Massachusetts Institute of Technology.

\footnotetext{
${ }^{7}$ (a) Bruce, M. I.; Hameister, C.; Swincer, A. G.; Wallis, R. C. Inorganic Syntheses 1982, 21, 78-83. Also, see: (b) Gilbert, J. D.; Wilkinson, G. J. Chem. Soc. (A) 1969, 1749, (c) Blackmore, T.; Bruce, M. I.; Stone, F. G. A. J. Chem. Soc. (A) 1971, 2376, and (d) Bruce, M. I.; Windsor, N. J., Aust. J. Chem. 1977, 30, 1601.

${ }^{8}$ (a) Walker, S. D.; Barder, T. E.; Martinelli, J. R.; Buchwald, S. L. Angew. Chem. Int. Ed. 2004, 43, 1871. (b) Tomori, H.; Fox, J. M.; Buchwald, S. L. J. Org. Chem. 2000, 65, 5334-5341.

${ }^{9}$ Enda, J.; Kuwajima, I. J. Am. Chem. Soc. 1985, 107, 5495-5501.
} 
Supplementary notes on the single-step conversion of amides to the corresponding alkynyl mines:

- The choice of 2-chloropyridine as the base additive was particularly effective as the use of 2,6-lutidine, $\mathrm{Et}_{3} \mathrm{~N}$, and ${ }^{i} \mathrm{Pr}_{2} \mathrm{NEt}$, in place of 2-chloropyridine under identical conditions (4.0 equiv of base) gave markedly diminished yield of the desired alkynyl imine 3a (Table S1).

- The use of $300-\mathrm{mol} \%$ of 2-chloropyridine gave satisfactory results ( $92 \%$ isolated yield of product 3a), while further decrease in the amount of 2-chloropyridine led to significantly lower yield of product 3a (i.e., Table S1, entry 7).

- The optimal solvent for activation of amides was found to be dichloromethane as

Table S1.
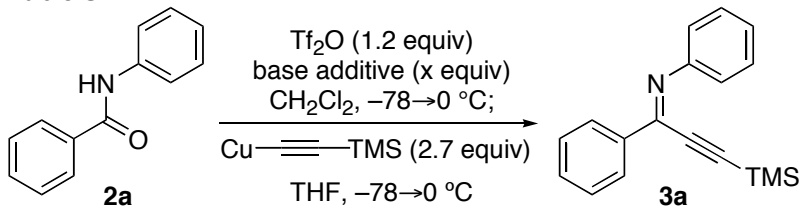

\begin{tabular}{cccc}
\hline Entry & Base Additive & $\mathrm{x}$ & Yield (\%) $^{\mathrm{a}}$ \\
\hline 1 & pyridine & 4 & $<10$ \\
2 & 2,6 -lutidine & 4 & 27 \\
3 & $\mathrm{Et}_{3} \mathrm{~N}$ & 4 & $<4^{\mathrm{b}}$ \\
4 & ${ }^{i} \mathrm{Pr}_{2} \mathrm{NEt}$ & 4 & $<20$ \\
5 & 2-chloropyridine & 4 & 97 \\
6 & 2-chloropyridine & 3 & 92 \\
7 & 2-chloropyridine & 2 & 65 \\
\hline
\end{tabular}

a Mass balance is the starting amide. ${ }^{\mathrm{b}}$ Mixture of products; no recovered SM. compared to other solvents examined (i.e., diethyl ether, tetrahydrofuran, butyl methyl ether, toluene, acetonitrile, nitromethane, 1,2dichloroethane).

- The use of copper acetylide provided the highest yield of the desired product as compared to the use of lithium acetylide, bromomagnesium acetylide, dichlorocerium acetylide, or cuprate derivatives under identical conditions. Approaches based on Sonogashira coupling after activation as described above provided the product 3a in poor yield $(<50 \%)$ and were not compatible with sensitive substrates (text ref. 8).

- With the exception of alkynyl imine in entry 15 of Table 1 , all alkynyl imines described below could be stored for weeks under an argon atmosphere at $0{ }^{\circ} \mathrm{C}$.

- A uniform and optimized set of conditions was used for all entries discussed in the text; please see experimental procedures for details.

Supplementary notes on the metal catalyzed cycloisomerization of alkynyl imines:

- The combination of commercially available $\mathrm{CpRu}\left(\mathrm{PPh}_{3}\right)_{2} \mathrm{Cl}(10 \mathrm{~mol} \%)$ and $\mathrm{SPhos}(10 \mathrm{~mol} \%)$ in the presence of a stoichiometric quantity of ammonium hexafluorophosphate (1.0 equiv) provided a highly effective catalyst system for the clean desilylation and cycloisomerization of alkynyl imines (Tables S2 and 1).

- The use of other additives in place of ammonium hexafluorophosphate (i.e., trifluoroethanol, ${ }^{i}$ propanol, ${ }^{i}$ propanol- $\mathrm{F}_{6}$, ${ }^{t}$ butanol, ammonium tetrafluoroborate, tetrabutylammonium fluoride, triethylamine trihydrogen-fluoride, and potassium fluoride) was found to be markedly less effective in providing cycloisomerization product 1a (Table 1, entry 1).

- Lower catalyst loadings resulted in slower conversion of starting alkynyl imine to the corresponding azaheterocycle (i.e., entry 39, Table S2).

- Attempted crystallization or direct spectroscopic detection of a CpRu-SPhos derived complex under a variety of conditions has not been successful thus far.

- The cycloisomerization of the C-desilylated alkynyl imine 3a under the optimized conditions was more rapid, but gave poor mass balance. The desilylation and cycloisomerization of alkynyl imine 3a required at least $7 \mathrm{~h}$. Complete conversion within $19 \mathrm{~h}$ was observed for all entries in Table 1. 


\begin{tabular}{|c|c|c|c|c|}
\hline & $\begin{array}{r}\begin{array}{r}\text { preca } \\
\text { phos }\end{array} \\
\begin{array}{r}\mathrm{NH} \\
\text { tol }\end{array}\end{array}$ & $\begin{array}{l}\text { atalyst (10 mol\%) } \\
\text { sphine (x mol\%) } \\
\underset{\mathrm{H}_{4} \mathrm{PF}_{6}(1 \text { equiv) }}{\text { oluene, } 105^{\circ} \mathrm{C}} \\
19 \mathrm{~h}\end{array}$ & & \\
\hline Entry & Precatalyst & Phosphine & $x$ & Yield (\%) ${ }^{a}$ \\
\hline 1 & $\mathrm{InBr}_{3}$ & - & - & 0 \\
\hline 2 & $\ln (\mathrm{OTf})_{3}$ & - & - & 0 \\
\hline 3 & $\mathrm{Sc}(\mathrm{OTf})_{3}$ & - & - & 0 \\
\hline 4 & $\mathrm{Pd}(\mathrm{OAc})_{2}$ & - & - & $<3$ \\
\hline 5 & $\mathrm{Pd}(\mathrm{OAc})_{2}$ & - & - & $<6^{b}$ \\
\hline 6 & $\mathrm{Pd}(\mathrm{OAc})_{2}$ & - & - & $<4^{c}$ \\
\hline 7 & $\mathrm{Pd}(\mathrm{OAc})_{2}$ & DPhos & 10 & 0 \\
\hline 8 & $\mathrm{Pd}(\mathrm{OAc})_{2}$ & XPhos & 10 & $<4$ \\
\hline 9 & $\mathrm{Pd}(\mathrm{OAc})_{2}$ & SPhos & 10 & $<3$ \\
\hline 10 & $\mathrm{~K}_{2} \mathrm{PtCl}_{4}$ & - & - & 0 \\
\hline 11 & $\mathrm{~K}_{2} \mathrm{PtCl}_{4}$ & - & - & $0^{b}$ \\
\hline 12 & $(\mathrm{RhClCOD})_{2}$ & - & - & 0 \\
\hline 13 & $\mathrm{RhCl}\left(\mathrm{Ph}_{3} \mathrm{P}\right)_{2} \mathrm{CO}$ & - & - & 0 \\
\hline 14 & {$\left[\mathrm{Ru}(p \text {-cymene }) \mathrm{Cl}_{2}\right]_{2}$} & - & - & $<2$ \\
\hline 15 & $\mathrm{CpRu}(\mathrm{dppf}) \mathrm{Cl}$ & - & - & 0 \\
\hline 16 & $\mathrm{CpRu}\left[(\mathrm{EtO})_{3} \mathrm{P}\right]_{2} \mathrm{Cl}$ & - & - & $<2$ \\
\hline 17 & $\mathrm{CpRu}\left[\left(p-\mathrm{MeOC}_{6} \mathrm{H}_{4}\right)_{3} \mathrm{P}\right]_{2} \mathrm{Cl}$ & I - & - & 36 \\
\hline 18 & $\mathrm{CpRu}\left(\mathrm{Me}_{3} \mathrm{P}\right)_{2} \mathrm{Cl}$ & - & - & $<1^{d}$ \\
\hline 19 & $\mathrm{CpRu}\left(\mathrm{Me}_{3} \mathrm{P}\right)_{2} \mathrm{Cl}$ & DPhos & 10 & $\leq 10^{d}$ \\
\hline 20 & $\mathrm{CpRu}\left(\mathrm{Me}_{3} \mathrm{P}\right)_{2} \mathrm{Cl}$ & XPhos & 10 & 19 \\
\hline 21 & $\mathrm{CpRu}\left(\mathrm{Me}_{3} \mathrm{P}\right)_{2} \mathrm{Cl}$ & SPhos & 10 & 19 \\
\hline 22 & {$\left[\mathrm{Ru}(\mathrm{COD}) \mathrm{Cl}_{2}\right]_{\mathrm{n}}$} & - & - & 0 \\
\hline 23 & {$\left[\mathrm{Ru}(\mathrm{COD}) \mathrm{Cl}_{2}\right]_{\mathrm{n}}$} & SPhos & 10 & 0 \\
\hline 24 & $\mathrm{CpRu}(\mathrm{COD}) \mathrm{Cl}$ & - & - & 0 \\
\hline 25 & $\mathrm{CpRu}(\mathrm{COD}) \mathrm{Cl}$ & SPhos & 10 & $<6$ \\
\hline 26 & $\mathrm{CpRu}(\mathrm{COD}) \mathrm{Cl}$ & SPhos & 20 & $<9$ \\
\hline 27 & $\mathrm{CpRu}(\mathrm{COD}) \mathrm{Cl}$ & $\mathrm{Ph}_{3} \mathrm{P}$ & 10 & 38 \\
\hline 28 & $\mathrm{CpRu}(\mathrm{COD}) \mathrm{Cl}$ & $\mathrm{Ph}_{3} \mathrm{P}$ & 15 & 62 \\
\hline 29 & $\mathrm{CpRu}(\mathrm{COD}) \mathrm{Cl}$ & $\mathrm{Ph}_{3} \mathrm{P}$ & 20 & 77 \\
\hline 30 & $\mathrm{CpRu}(\mathrm{COD}) \mathrm{Cl}$ & $(2 \text {-furyl })_{3} \mathrm{P}$ & 15 & 36 \\
\hline 31 & $\mathrm{CpRu}(\mathrm{COD}) \mathrm{Cl}$ & $(2 \text {-furyl })_{3} \mathrm{P}, \mathrm{Ph}_{3} \mathrm{P}$ & 10,10 & 67 \\
\hline 32 & $\mathrm{CpRu}(\mathrm{COD}) \mathrm{Cl}$ & $(2 \text {-furyl })_{3} P$, SPhos & 10,10 & 54 \\
\hline 33 & $\mathrm{CpRu}(\mathrm{COD}) \mathrm{Cl}$ & $\mathrm{Ph}_{3} \mathrm{P}$, SPhos & 13,13 & 88 \\
\hline 34 & $C p R u(C O D) C l$ & $\mathrm{Ph}_{3} P, \mathrm{SPhos}$ & 15,15 & 97 \\
\hline 35 & $\mathrm{CpRu}\left(\mathrm{Ph}_{3} \mathrm{P}\right)_{2} \mathrm{Cl}$ & - & - & 80 \\
\hline 36 & $\mathrm{CpRu}\left(\mathrm{Ph}_{3} \mathrm{P}\right)_{2} \mathrm{Cl}$ & DPhos & 10 & 30 \\
\hline 37 & $\mathrm{CpRu}\left(\mathrm{Ph}_{3} \mathrm{P}\right)_{2} \mathrm{Cl}$ & XPhos & 10 & 74 \\
\hline 38 & $\mathrm{CpRu}\left(\mathrm{Ph}_{3} \mathrm{P}\right)_{2} \mathrm{Cl}$ & SPhos & 10 & 95 \\
\hline 39 & $\mathrm{CpRu}\left(\mathrm{Ph}_{3} \mathrm{P}\right)_{2} \mathrm{Cl}$ & SPhos & 5 & 56 \\
\hline 40 & $\mathrm{CpRu}\left(\mathrm{Ph}_{3} \mathrm{P}\right)_{2} \mathrm{Cl}$ & $\mathrm{Ph}_{3} \mathrm{P}$ & 10 & 80 \\
\hline 41 & $\mathrm{CpRu}\left(\mathrm{Ph}_{3} \mathrm{P}\right)_{2} \mathrm{Cl}$ & $\mathrm{Ph}_{3} \mathrm{P}$ & 20 & 81 \\
\hline 42 & $\mathrm{CpRu}\left(\mathrm{Ph}_{3} \mathrm{P}\right)_{2} \mathrm{Cl}$ & $(2 \text {-furyl })_{3} \mathrm{P}$ & 10 & 76 \\
\hline
\end{tabular}

${ }^{a}$ Mass balance is the starting silylated alkynyl imine $3 a$. ${ }^{b_{2}} 2.2$ mol\% TFA additive.

${ }^{\mathrm{c}} 10 \mathrm{~mol} \%$ AgOAc additive. ${ }^{\mathrm{d} S a m e}$ result at $75^{\circ} \mathrm{C}$. ${ }^{\mathrm{e}} 5 \mathrm{~mol} \%$ Ru-complex.

Structures of DPhos, XPhos, and SPhos are shown in Figure S1. ${ }^{8}$

Figure S1.

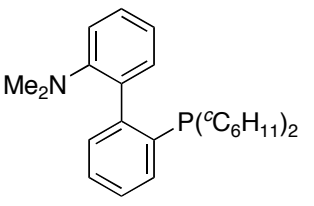

DPhos

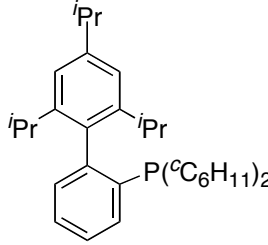

XPhos<smiles>COc1cccc(OC)c1-c1ccccc1</smiles>

SPhos 
- To evaluate a potential loss of catalyst activity due to trace $\mathrm{Cu}$-contamination from the imine synthesis step, necessitating the use of $10 \mathrm{~mol} \% \mathrm{Ru}$-catalyst, an experiment based on the standard conditions (Table 1 , entry 1) was conducted containing additional $\mathrm{CuBr} \cdot \mathrm{SMe}_{2}(5$ mol\%). No change was observed and complete conversion was seen within the normal $7 \mathrm{~h}$ period for this substrate affording the product 1a in $90 \%$ yield.

- When the conversion of 3a to $\mathbf{1 a}$ was conducted with $100 \mathrm{~mol} \%$ of product $\mathbf{1 a}$ present at the start of the experiment (under optimal conditions; i.e., Table 1, entry 1) the conversion to $\mathbf{1 a}$ was not complete even after $22 \mathrm{~h}$ (typically complete in $7 \mathrm{~h}$ for $\mathbf{3 a}$ ) providing $1 \mathrm{a}$ in $79 \%$ yield with the remaining substrate $\mathbf{3 a}$ accounting for the mass balance.

- Due to ready availability of both precatalyst and ligand, a uniform and optimum set of conditions using $10 \mathrm{~mol} \%$ of each was used for all entries (except entry 16) in Table 1.

\section{Supplementary notes on the deuterium labeling experiments:}

- The use of ammonium hexafluorophosphate- $d_{4}(>95 \%, 1.0$ equiv) under optimal conditions for cycloisomerization of alkynyl imine 3a (1 equiv) provided the corresponding quinoline 1a- $d_{1}$ in $89 \%$ yield with $68 \%$ d-incorporation at C3 and $7 \%$ d-incorporation at C4 (Scheme S1).

- Similarly, the cycloisomerization of alkynyl imine 3a- $d_{5}(98 \%, 1$ equiv) in the presence of ammonium hexafluorophosphate (1.0 equiv) afforded the corresponding quinoline $\mathbf{1 a}-d_{5}$ in $76 \%$ yield with $2 \%$ d-incorporation at $\mathrm{C} 3$ and $68 \%$ d-incorporation at $\mathrm{C} 4$ (Scheme $\mathrm{S} 1$ ).

- Additionally, C-desilylation of alkynyl imine 3a and complete deuterium incorporation at the terminal alkyne gave the azadienyne derivative $4 \mathbf{a}-d_{1}(>95 \%)$ that was converted to the quinoline 1a- $d_{1}$ in $52 \%$ yield with $72 \%$ d-incorporation at $\mathrm{C} 3$ and $7 \%$ d-incorporation at $\mathrm{C} 4$ without the need for ammonium hexafluorophosphate (Scheme S1). As anticipated the conversion of alkynyl imine $\mathbf{4 a}-d_{1}$ to the desired product was significantly faster as compared to C-silylated alkynyl imine $\mathbf{3 a}$ at the expense of poor mass recovery and lower yield.

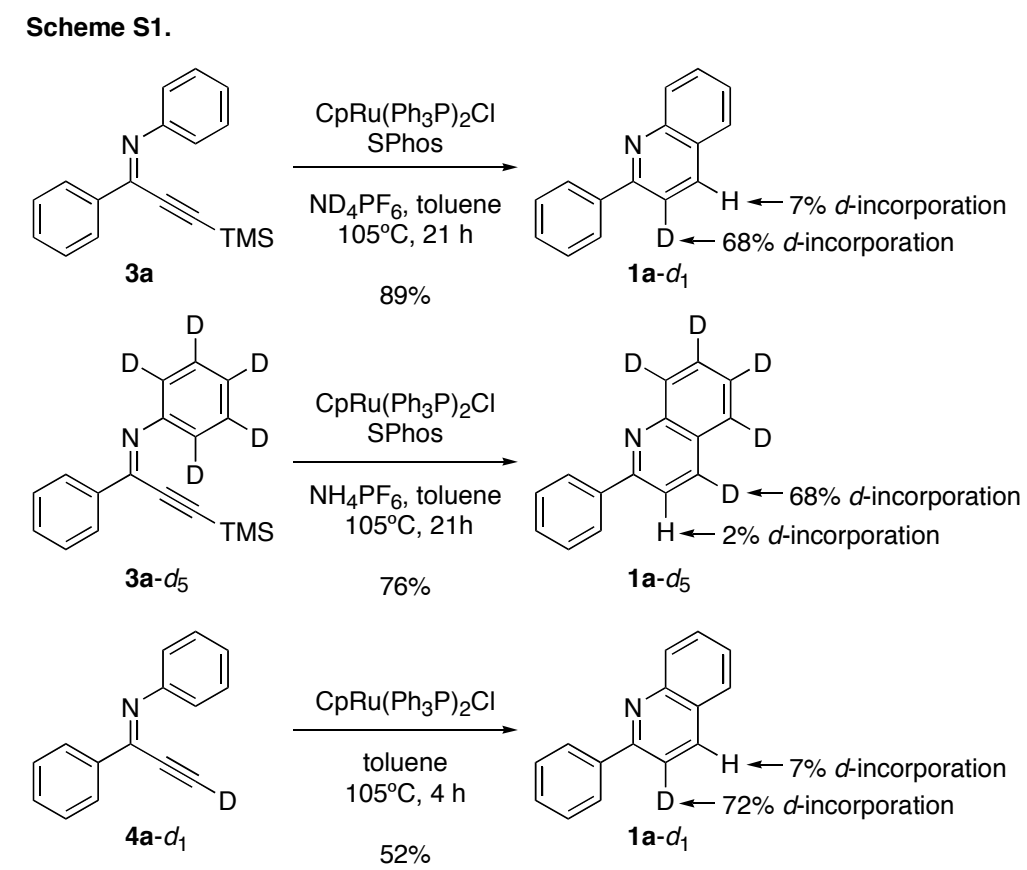

- The results are most consistent with the intermediacy of a ruthenium vinylidene 8 (Scheme 3) prior to cycloisomerization step. Incomplete deuterium incorporation at the expected site and leakage to the alternate position $(2-7 \%$, Scheme S1) may in part be due to an intermolecular scrambling after cycloisomerization of $\mathbf{8}$ and prior to release of product $\mathbf{1}$ (i.e., the intermediate 9 or the metal hydride tautomer in Scheme 3). 


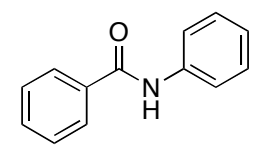

$2 \mathbf{a}$

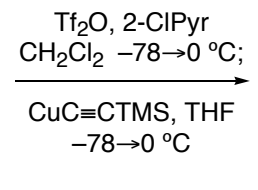

$97 \%$

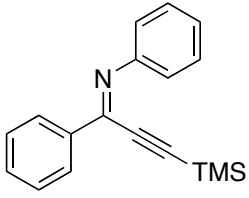

$3 a$

\section{N-Phenyl-2-phenyl-4-trimethylsilyl-1-azabut-1-en-3-yne (3a, Table 1, entry 1):}

Trifluoromethanesulfonic anhydride $(2.51 \mathrm{~mL}, 15.2 \mathrm{mmol}, 1.20$ equiv) was added via syringe over $1 \mathrm{~min}$ to a stirred mixture of amide $2 \mathrm{a}(2.50 \mathrm{~g}, 12.7 \mathrm{mmol}, 1$ equiv) and 2-chloropyridine (4.81 $\mathrm{mL}, 50.7 \mathrm{mmol}, 4.00$ equiv) in $\mathrm{CH}_{2} \mathrm{Cl}_{2}(25 \mathrm{~mL})$ at $-78{ }^{\circ} \mathrm{C}$. After $5 \mathrm{~min}$, the reaction mixture was warmed to $0{ }^{\circ} \mathrm{C}$. After $20 \mathrm{~min}$, the solution was cooled to $-78{ }^{\circ} \mathrm{C}$ and a freshly prepared solution of copper (I) (trimethylysilyl)-acetylide (5.50 g, $34.2 \mathrm{mmol}, 2.70$ equiv) in THF $(60 \mathrm{~mL})$ at $0{ }^{\circ} \mathrm{C}$ was added via cannula. The reaction mixture was kept at $-78{ }^{\circ} \mathrm{C}$ for $5 \mathrm{~min}$ and then warmed to $0{ }^{\circ} \mathrm{C}$. After $10 \mathrm{~min}$, the crude reaction mixture was filtered through celite $(2 \mathrm{~cm}$ diam. $\times 3 \mathrm{~cm} \mathrm{ht}$. $)$ and the filtrate was concentrated under reduced pressure. The residue was purified by flash column chromatography on silica gel $\left(100 \%\right.$ hexanes $\rightarrow 7 \%$ EtOAc/hexanes) to give the alkynyl imine $\mathbf{3} \mathbf{a}^{10}$ as a yellow oil (3.42 g, 97\%).

${ }^{1} \mathrm{H}$ NMR $\left(500 \mathrm{MHz}, \mathrm{CDCl}_{3}, 20{ }^{\circ} \mathrm{C}\right) \delta: \quad 8.21-8.18(\mathrm{~m}, 2 \mathrm{H}, \operatorname{ArH}(o-\mathrm{C}=\mathrm{N})), 7.51-7.45(\mathrm{~m}, 3 \mathrm{H}$, ArH), 7.40-7.36 (m, 2H, ArH), $7.17(\mathrm{tt}, 1 \mathrm{H}, J=7.5,1.1$ $\mathrm{Hz}, \operatorname{ArH}), 7.14-7.11(\mathrm{~m}, 2 \mathrm{H}, \operatorname{ArH}), 0.14$ (s, 9H, $\left.\mathrm{Si}\left(\mathrm{CH}_{3}\right)_{3}\right)$.

${ }^{13} \mathrm{C}$ NMR $\left(125 \mathrm{MHz}, \mathrm{CDCl}_{3}, 20^{\circ} \mathrm{C}\right) \delta:$

FTIR (neat) $\mathrm{cm}^{-1}$ :

HRMS (ESI):

Analysis

TLC (20\% EtOAc/hexanes), Rf:
$151.7,150.1,137.0,131.4,128.6,128.5,128.3,125.0$, $120.9,105.4,97.5,-0.5$.

3063 (m), 3030 (w), 2960 (m), 1588 (s, C=N), 1565 (s).

calcd for $\mathrm{C}_{18} \mathrm{H}_{20} \mathrm{NSi}[\mathrm{M}+\mathrm{H}]^{+}:$278.1360, found: 278.1365 .

calcd for $\mathrm{C}_{18} \mathrm{H}_{19} \mathrm{NSi}$ : C, 77.93; H, 6.90; N, 5.05, found: $\mathrm{C}, 77.97 ; \mathrm{H}, 6.87 ; \mathrm{N}, 5.10$.

0.59 (UV, CAM).

\footnotetext{
${ }^{10}$ For a prior synthesis (characterization data not listed), see: Ito, Y.; Inouye, M.; Murakami, M. Chem. Lett. 1989, 7, $1261-1264$.
} 


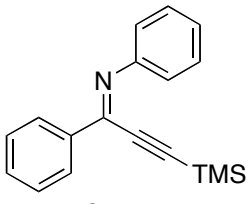

3a

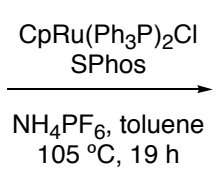

$90 \%$

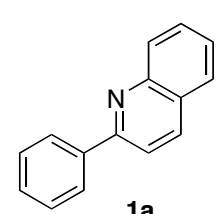

$1 a$

\section{2-Phenylquinoline (1a, Table 1, entry 1):}

An oven dried pressure vessel containing a magnetic stir bar was charged with ammonium hexafluorophosphate (587 mg, $3.60 \mathrm{mmol}, 1.00$ equiv), $\mathrm{CpRuCl}\left(\mathrm{PPh}_{3}\right)_{2}(262 \mathrm{mg}, 0.35 \mathrm{mmol}, 0.10$ equiv) and SPhos (148 mg, $0.35 \mathrm{mmol}, 0.10$ equiv) under a nitrogen atmosphere in a glove-box and the flask sealed and brought out of the glove-box. Imine 3a (1.00 g, $3.60 \mathrm{mmol}, 1$ equiv) and toluene $(18 \mathrm{~mL})$ were subsequently added via syringe. The flask was flushed with argon, sealed, stirred, and placed in an oil bath at $105^{\circ} \mathrm{C}$. After $19 \mathrm{~h}$, the reaction vessel was allowed to cool to ambient temperature and the mixture was transferred to a recovery flask with a $20-\mathrm{mL}$ portion of dichloromethane. This solution was concentrated under reduced pressure and the residue was purified by flash column chromatography on silica gel $(15 \rightarrow 50 \%$ EtoAc/Hexanes) to afford the quinoline $\mathbf{1 a}^{11}$ as a pale yellow solid (668 $\left.\mathrm{mg}, 90 \%\right)$.

${ }^{1} \mathrm{H}$ NMR $\left(500 \mathrm{MHz}, \mathrm{CDCl}_{3}, 20{ }^{\circ} \mathrm{C}\right) \delta: \quad 8.25(\mathrm{~d}, 1 \mathrm{H}, J=8.5 \mathrm{~Hz}, \mathrm{ArH}), 8.21-8.16(\mathrm{~m}, 3 \mathrm{H}, \operatorname{ArH})$, $7.90(\mathrm{~d}, 1 \mathrm{H}, J=8.5 \mathrm{~Hz}, \operatorname{ArH}), 7.85(\mathrm{~d}, 1 \mathrm{H}, J=8.2 \mathrm{~Hz}$, ArH), 7.75 (ddd, 1H, $J=8.5,7.0,1.5 \mathrm{~Hz}, \operatorname{ArH}), 7.57-$ $7.52(\mathrm{~m}, 3 \mathrm{H}, \mathrm{ArH}), 7.48(\mathrm{tt}, 1 \mathrm{H}, J=7.3,1.2 \mathrm{~Hz}, \operatorname{ArH})$.

${ }^{13} \mathrm{C} \mathrm{NMR}\left(125 \mathrm{MHz}, \mathrm{CDCl}_{3}, 20{ }^{\circ} \mathrm{C}\right) \delta: \quad 157.5,148.4,139.8,137.0,129.9,129.9,129.5,129.0$, $127.8,127.7,127.3,126.5,119.2$.

FTIR (neat) $\mathrm{cm}^{-1}$ :

3189 (s), 3055 (w), 2091 (s), 1617 (w), 1597 (s), 1491 (m), 1447 (s).

HRMS (EI):

calcd for $\mathrm{C}_{15} \mathrm{H}_{11} \mathrm{~N}[\mathrm{M}]^{+}: 205.0886$, found: 205.0885 .

TLC (20\% EtOAc-hexanes), Rf: $\quad 0.51$ (UV, CAM).

\footnotetext{
${ }^{11}$ Sangu, K.: Fuchibe, K.: Akiyama, T. Org. Lett. 2004, 6, 353-355.
} 


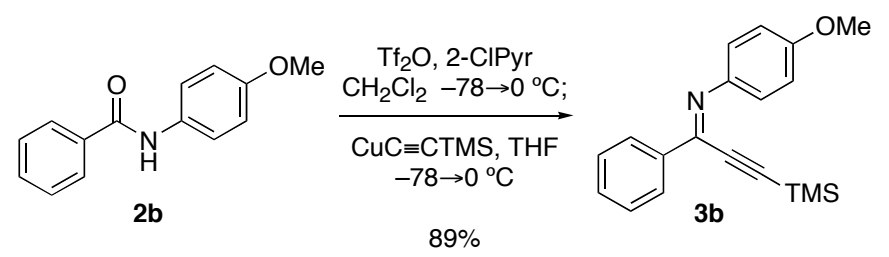

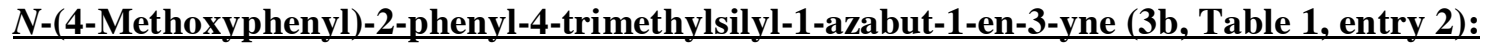

Trifluoromethanesulfonic anhydride $(174 \mu \mathrm{L}, 1.06 \mathrm{mmol}, 1.20$ equiv) was added via syringe over $1 \mathrm{~min}$ to a stirred mixture of amide $\mathbf{2 b}(200 \mathrm{mg}, 0.88 \mathrm{mmol}, 1$ equiv) and 2-chloropyridine (333 $\mu \mathrm{L}, 3.52 \mathrm{mmol}, 4.00$ equiv) in $\mathrm{CH}_{2} \mathrm{Cl}_{2}(1.8 \mathrm{~mL})$ at $-78^{\circ} \mathrm{C}$. After $5 \mathrm{~min}$, the reaction mixture was warmed to $0{ }^{\circ} \mathrm{C}$. After $20 \mathrm{~min}$, the solution was cooled to $-78{ }^{\circ} \mathrm{C}$ and a freshly prepared solution of copper (I) (trimethylysilyl)-acetylide (383 mg, $2.38 \mathrm{mmol}, 2.70$ equiv) in THF $(5.0 \mathrm{~mL})$ at $0{ }^{\circ} \mathrm{C}$ was added via cannula. The reaction mixture was kept at $-78^{\circ} \mathrm{C}$ for $5 \mathrm{~min}$ and then warmed to $0{ }^{\circ} \mathrm{C}$. After $10 \mathrm{~min}$, the crude reaction mixture was filtered through celite $(2 \mathrm{~cm}$ diam. $\times 3 \mathrm{~cm} \mathrm{ht}$.) and the filtrate was concentrated under reduced pressure. The residue was purified by flash column chromatography on silica gel ( $100 \%$ hexanes $\rightarrow 10 \%$ EtOAc/hexanes) to afford the alkynyl imine $\mathbf{3 b}$ as a yellow oil $(240 \mathrm{mg}, 89 \%)$.

${ }^{1} \mathrm{H}$ NMR $\left(500 \mathrm{MHz}, \mathrm{CDCl}_{3}, 2{ }^{\circ} \mathrm{C}\right) \delta$ :

${ }^{13} \mathrm{C}$ NMR $\left(125 \mathrm{MHz}, \mathrm{CDCl}_{3}, 20{ }^{\circ} \mathrm{C}\right) \delta$ :

FTIR $\left(\mathrm{CDCl}_{3}\right) \mathrm{cm}^{-1}$ :

HRMS (ESI):

TLC (20\% EtOAc/hexanes), Rf:
8.21-8.15 (m, 2H, ArH $(o-\mathrm{C}=\mathrm{N})), 7.50-7.42(\mathrm{~m}, 3 \mathrm{H}$, $\operatorname{ArH}(m-\mathrm{C}=\mathrm{N}, p-\mathrm{C}=\mathrm{N})), 7.31-7.25(\mathrm{~m}, 2 \mathrm{H}, \mathrm{ArH}), 6.96-$ $6.90(\mathrm{~m}, 2 \mathrm{H}, \mathrm{ArH}), 3.83$ (s, 3H, $\left.\mathrm{CH}_{3} \mathrm{O}\right), 0.21(\mathrm{~s}, 9 \mathrm{H}$, $\left.\mathrm{Si}\left(\mathrm{CH}_{3}\right)_{3}\right)$.

157.7, 148.1, 144.2, 137.6, 131.0, 128.5, 128.1, 123.3, $113.7,104.8,98.1,55.6,-0.4$.

$3066(\mathrm{w}), 2962(\mathrm{~m}), 2838(\mathrm{w}), 1605(\mathrm{~m}, \mathrm{C}=\mathrm{N}), 1559$

(m), 1503 (s), 1251 (s).

calcd for $\mathrm{C}_{19} \mathrm{H}_{22} \mathrm{NOSi}[\mathrm{M}+\mathrm{H}]^{+}: 308.1465$, found: 308.1472 .

0.49 (UV, CAM). 


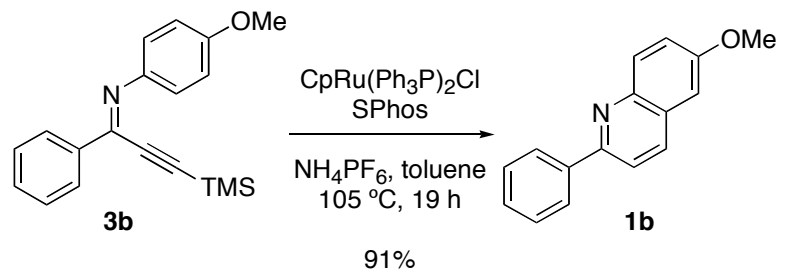

\section{6-Methoxy-2-phenylquinoline (1b, Table 1, entry 2):}

An oven dried pressure vessel containing a magnetic stir bar was charged with ammonium hexafluorophosphate (40 mg, $0.24 \mathrm{mmol}, 1.0$ equiv), $\mathrm{CpRuCl}\left(\mathrm{PPh}_{3}\right)_{2}$ (18 mg, $0.024 \mathrm{mmol}, 0.10$ equiv) and SPhos (10 mg, $0.024 \mathrm{mmol}, 0.10$ equiv) under a nitrogen atmosphere in a glove-box and the flask sealed and brought out of the glove-box. Imine $\mathbf{3 b}(75 \mathrm{mg}, 0.24 \mathrm{mmol}, 1$ equiv) and toluene $(1.2 \mathrm{~mL})$ were subsequently added via syringe. The flask was flushed with argon, sealed, stirred, and placed in an oil bath at $105^{\circ} \mathrm{C}$. After $19 \mathrm{~h}$, the reaction vessel was allowed to cool to ambient temperature and the mixture was transferred to a recovery flask with a $10-\mathrm{mL}$ portion of dichloromethane. This solution was concentrated under reduced pressure and the residue was purified by flash column chromatography on silica gel $\left(20 \%\right.$ EtoAc/Hexanes) to afford the quinoline $\mathbf{1 b}^{11}$ as a pale yellow solid (52 mg, 91\%).

${ }^{1} \mathrm{H}$ NMR $\left(500 \mathrm{MHz}, \mathrm{CDCl}_{3}, 20^{\circ} \mathrm{C}\right) \delta: \quad 8.16-8.10(\mathrm{~m}, 3 \mathrm{H}, \mathrm{ArH}), 8.08(\mathrm{~d}, 1 \mathrm{H}, J=9.2 \mathrm{~Hz}, \operatorname{ArH})$, $7.85(\mathrm{~d}, 1 \mathrm{H}, J=8.9 \mathrm{~Hz}, \operatorname{ArH}), 7.56-7.50(\mathrm{~m}, 2 \mathrm{H}, \operatorname{ArH})$, $7.48-7.42(\mathrm{~m}, 1 \mathrm{H}, \mathrm{ArH}), 7.40(\mathrm{dd}, 1 \mathrm{H}, J=9.2,2.7 \mathrm{~Hz}$, $\left.\operatorname{ArH~}\left(\mathrm{CH}_{3} \mathrm{OCCH}\right)\right), 7.11(\mathrm{~d}, 1 \mathrm{H}, J=3.1 \mathrm{~Hz}$, $\left.\mathrm{ArH}\left(\mathrm{CH}_{3} \mathrm{OCCH}\right)\right), 3.99\left(\mathrm{~s}, 3 \mathrm{H}, \mathrm{CH}_{3} \mathrm{O}\right)$.

${ }^{13} \mathrm{C}$ NMR $\left(125 \mathrm{MHz}, \mathrm{CDCl}_{3}, 20^{\circ} \mathrm{C}\right) \delta:$

FTIR (neat) $\mathrm{cm}^{-1}$ :

HRMS (EI):

TLC (20\% EtOAc-hexanes), Rf:
$157.8,155.2,144.5,139.9,135.7,131.3,129.1,129.0$, $128.3,127.5,122.5,119.4,105.1,55.7$.

$3057(\mathrm{w}), 2958(\mathrm{~m}), 2836(\mathrm{w}), 1621(\mathrm{~m}), 1599(\mathrm{~m})$, 1493 (s).

calcd for $\mathrm{C}_{16} \mathrm{H}_{13} \mathrm{NO}[\mathrm{M}]^{+}$: 235.0992, found: 235.0984.

0.39 (UV, CAM). 

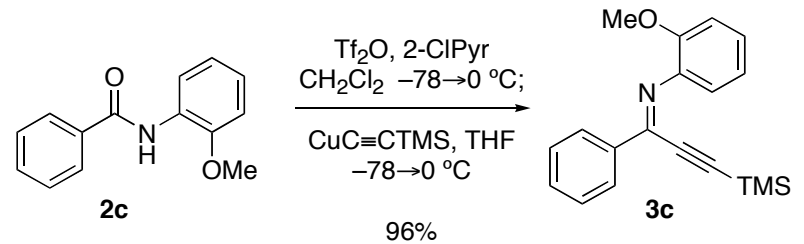

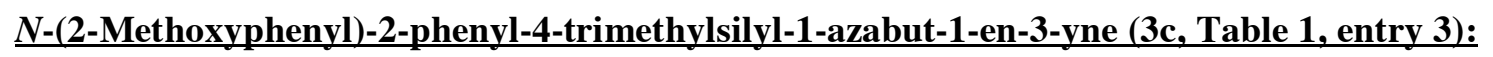

Trifluoromethanesulfonic anhydride $(218 \mu \mathrm{L}, 1.32 \mathrm{mmol}, 1.20$ equiv) was added via syringe over $1 \mathrm{~min}$ to a stirred mixture of amide $2 \mathrm{c}(250 \mathrm{mg}, 1.10 \mathrm{mmol}, 1$ equiv) and 2-chloropyridine (416 $\mu \mathrm{L}, 4.40 \mathrm{mmol}, 4.00$ equiv) in $\mathrm{CH}_{2} \mathrm{Cl}_{2}(2.2 \mathrm{~mL})$ at $-78^{\circ} \mathrm{C}$. After $5 \mathrm{~min}$, the reaction mixture was warmed to $0{ }^{\circ} \mathrm{C}$. After $20 \mathrm{~min}$, the solution was cooled to $-78{ }^{\circ} \mathrm{C}$ and a freshly prepared solution of copper (I) (trimethylysilyl)-acetylide (477 mg, $2.97 \mathrm{mmol}, 2.70$ equiv) in THF $(5.0 \mathrm{~mL})$ at $0{ }^{\circ} \mathrm{C}$ was added via cannula. The reaction mixture was kept at $-78{ }^{\circ} \mathrm{C}$ for $5 \mathrm{~min}$ and then warmed to $0{ }^{\circ} \mathrm{C}$. After $10 \mathrm{~min}$, the crude reaction mixture was filtered through celite $(2 \mathrm{~cm}$ diam. $\times 3 \mathrm{~cm} \mathrm{ht}$. $)$ and the filtrate was concentrated under reduced pressure. The residue was purified by flash column chromatography on silica gel $(100 \%$ hexanes $\rightarrow 10 \%$ EtOAc/hexanes) to afford the alkynyl imine 3c as a yellow oil (326 mg, 97\%).

${ }^{1} \mathrm{H}$ NMR $\left(500 \mathrm{MHz}, \mathrm{CDCl}_{3}, 20{ }^{\circ} \mathrm{C}\right) \delta:$

8.24-8.20 (m, 2H, ArH $(o-\mathrm{C}=\mathrm{N})), 7.52-7.44(\mathrm{~m}, 3 \mathrm{H}$, $\operatorname{ArH}(p-\mathrm{C}=\mathrm{N}, m-\mathrm{C}=\mathrm{N})), 7.14(\mathrm{ddd}, 1 \mathrm{H}, J=8.3,7.3,1.8$ $\mathrm{Hz}, \mathrm{ArH}), 7.01(\mathrm{dd}, 1 \mathrm{H}, J=7.6,2.1 \mathrm{~Hz}, \mathrm{ArH}), 6.98$ $6.94(\mathrm{~m}, 2 \mathrm{H}, \mathrm{ArH}), 3.85\left(\mathrm{~s}, 3 \mathrm{H}, \mathrm{OCH}_{3}\right), 0.11(\mathrm{~s}, 9 \mathrm{H}$, $\left.\mathrm{Si}\left(\mathrm{CH}_{3}\right)_{3}\right)$.

${ }^{13} \mathrm{C}$ NMR $\left(125 \mathrm{MHz}, \mathrm{CDCl}_{3}, 20{ }^{\circ} \mathrm{C}\right) \delta: \quad 151.6,150.5,141.5,136.8,131.3,128.4,128.4,125.7$, $120.8,120.5,111.5,104.9,97.7,55.9,-0.5$.

FTIR (neat) $\mathrm{cm}^{-1}$ :

3064 (w), 2959 (m), 2900 (w), 2834 (w), 1590 (s), 1567 (s), 1488 (s).

HRMS (ESI):

calcd for $\mathrm{C}_{19} \mathrm{H}_{22} \mathrm{NOSi}[\mathrm{M}+\mathrm{H}]^{+}:$308.1465, found: 308.1477 .

TLC (20\% EtOAc/hexanes), Rf:

$0.49\left(\mathrm{UV}, \mathrm{KMnO}_{4}\right)$. 

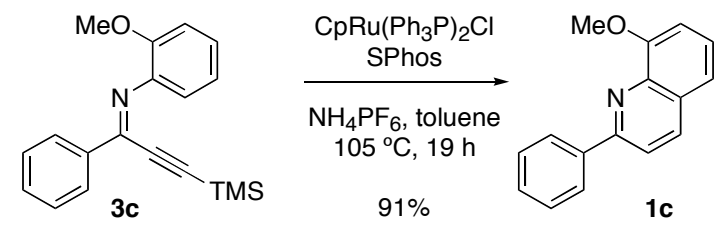

\section{8-Methoxy-2-phenylquinoline (1c, Table 1, entry 3):}

An oven dried pressure vessel containing a magnetic stir bar was charged with ammonium hexafluorophosphate (53 mg, $0.33 \mathrm{mmol}, 1.0$ equiv), $\mathrm{CpRuCl}\left(\mathrm{PPh}_{3}\right)_{2}$ (24 mg, $0.033 \mathrm{mmol}, 0.10$ equiv) and SPhos (13 mg, $0.033 \mathrm{mmol}, 0.10$ equiv) under a nitrogen atmosphere in a glove-box and the flask sealed and brought out of the glove-box. Imine $3 \mathbf{c}(100 \mathrm{mg}, 0.33 \mathrm{mmol}, 1$ equiv) and toluene (1.6 mL) were subsequently added via syringe. The flask was flushed with argon, sealed, stirred, and placed in an oil bath at $105^{\circ} \mathrm{C}$. After $19 \mathrm{~h}$, the reaction vessel was allowed to cool to ambient temperature and the mixture was transferred to a recovery flask with a $10-\mathrm{mL}$ portion of dichloromethane. This solution was concentrated under reduced pressure and the residue was purified by flash column chromatography on silica gel (20\% EtoAc/Hexanes) to afford the quinoline $1 \mathrm{c}^{12}$ as a pale yellow solid (70 mg, 91\%).

${ }^{1} \mathrm{H}$ NMR $\left(500 \mathrm{MHz}, \mathrm{CDCl}_{3}, 20^{\circ} \mathrm{C}\right) \delta:$

${ }^{13} \mathrm{C}$ NMR $\left(125 \mathrm{MHz}, \mathrm{CDCl}_{3}, 20^{\circ} \mathrm{C}\right) \delta$ :

FTIR (neat) $\mathrm{cm}^{-1}$ :

HRMS (ESI):

TLC (20\% EtOAc/hexanes), Rf:
8.24-8.18 (m, 3H, ArH), 7.93 (d, 1H, $J=8.5 \mathrm{~Hz}, \operatorname{ArH})$, $7.53(\mathrm{t}, 2 \mathrm{H}, J=7.5 \mathrm{~Hz}, \operatorname{ArH}), 7.49-7.41$ (m, 3H, ArH), 7.10 (dd, 1H, $J=7.6,1.1 \mathrm{~Hz}, \operatorname{ArH}), 4.13$ (s, 3H, OCH 3 ).

$156.5,155.7,140.3,140.0,137.0,129.4,129.0,128.5$, $127.9,126.7,119.7,119.5,108.2,56.3$.

3060 (m), 2934 (m), 2834 (w), 1599 (s), 1556 (s), 1467 (s), 1258 (s).

calcd for $\mathrm{C}_{16} \mathrm{H}_{14} \mathrm{NO}[\mathrm{M}+\mathrm{H}]^{+}:$236.1070, found: 236.1066 .

$0.23\left(\mathrm{UV}, \mathrm{KMnO}_{4}\right)$.

\footnotetext{
${ }^{12}$ For a prior synthesis (characterization data not listed), see: Collin, J.; Jaber, N.; Lannou, M. I. Tetrahedron Lett. 2001, $42,7405-7407$.
} 
<smiles>O=C(Nc1cccc(C(F)(F)F)c1)c1ccccc1</smiles>

2d

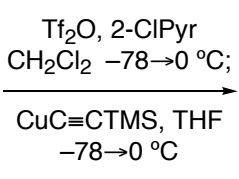

$73 \%$

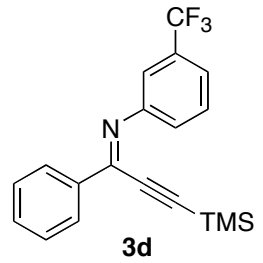

$3 d$

\section{$\underline{N \text {-(3-Trifluoromethylphenyl)-2-phenyl-4-trimethylsilyl-1-azabut-1-en-3-yne (3d, Table 1, entry 4): }}$}

Trifluoromethanesulfonic anhydride $(251 \mu \mathrm{L}, 1.52 \mathrm{mmol}, 1.20$ equiv) was added via syringe over $1 \mathrm{~min}$ to a stirred mixture of amide $2 \mathbf{d}$ (337 mg, $1.27 \mathrm{mmol}, 1$ equiv) and 2-chloropyridine (481 $\mu \mathrm{L}, 5.08 \mathrm{mmol}, 4.00$ equiv) in $\mathrm{CH}_{2} \mathrm{Cl}_{2}(2.5 \mathrm{~mL})$ at $-78{ }^{\circ} \mathrm{C}$. After $5 \mathrm{~min}$, the reaction mixture was warmed to $0{ }^{\circ} \mathrm{C}$. After $20 \mathrm{~min}$, the solution was cooled to $-78{ }^{\circ} \mathrm{C}$ and a freshly prepared solution of copper (I) (trimethylysilyl)-acetylide (552 mg, $3.43 \mathrm{mmol}, 2.70$ equiv) in THF $(7.0 \mathrm{~mL})$ at $0{ }^{\circ} \mathrm{C}$ was added via cannula. The reaction mixture was kept at $-78{ }^{\circ} \mathrm{C}$ for $5 \mathrm{~min}$ and then warmed to $0{ }^{\circ} \mathrm{C}$. After $10 \mathrm{~min}$, the crude reaction mixture was filtered through celite $(2 \mathrm{~cm} \mathrm{diam.} \times 3 \mathrm{~cm} \mathrm{ht}$. $)$ and the filtrate was concentrated under reduced pressure. The residue was purified by flash column chromatography on silica gel $(100 \%$ hexanes $\rightarrow 7.5 \%$ EtOAc/hexanes) to afford the alkynyl imine 3d as a pale yellow oil (321 mg, 73\%). Desilylation of 3d (see pages S19 and S38 for experimental procedures) afforded less than $10 \%$ yield of the corresponding terminal alkyne due to rapid nucleophilic addition (i.e., water and methanol) to this terminal alkynyl imine.

${ }^{1} \mathrm{H}$ NMR $\left(500 \mathrm{MHz}, \mathrm{CDCl}_{3}, 20^{\circ} \mathrm{C}\right) \delta: \quad 8.20-8.17(\mathrm{~m}, 2 \mathrm{H}, \operatorname{ArH}(o-\mathrm{C}=\mathrm{N})), 7.55-7.47(\mathrm{~m}, 4 \mathrm{H}$, ArH), 7.45-7.42 (m, 1H, ArH), 7.40 (s, 1H, ArH), 7.29$7.25(\mathrm{~m}, 1 \mathrm{H}, \mathrm{ArH}), 0.06\left(\mathrm{~s}, 9 \mathrm{H}, \mathrm{Si}\left(\mathrm{CH}_{3}\right)_{3}\right)$.

${ }^{13} \mathrm{C}$ NMR $\left(125 \mathrm{MHz}, \mathrm{CDCl}_{3}, 20^{\circ} \mathrm{C}\right) \delta:$

FTIR (neat) $\mathrm{cm}^{-1}$ :

HRMS (ESI):

TLC (20\% EtOAc/hexanes), Rf: $152.3,151.5,136.6,131.8,131.1(\mathrm{q}, J=32.0 \mathrm{~Hz}), 129.2$, $128.6,128.5,124.6$ (q, $J=271.9 \mathrm{~Hz}), 124.6,121.5$ (q, $J$ $=3.9 \mathrm{~Hz}), 117.8(\mathrm{q}, J=3.8 \mathrm{~Hz}), 106.8,96.9,-0.7$.

3067 (m), 2962 (m), 1589 (s, C=N), 1567 (s), 1330 (s).

calcd for $\mathrm{C}_{19} \mathrm{H}_{19} \mathrm{~F}_{3} \mathrm{NSi}[\mathrm{M}+\mathrm{H}]^{+}:$346.1233, found: 346.1236.

0.57 (UV, CAM). 

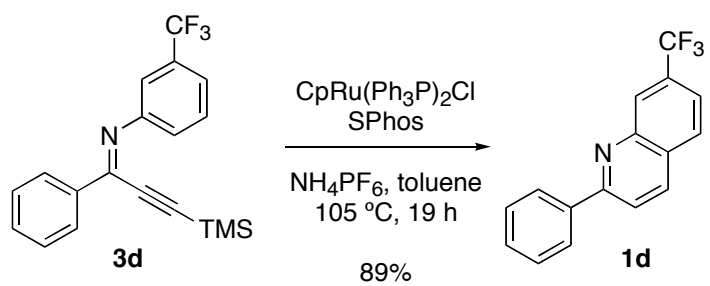

\section{2-Phenyl-7-trifluoromethyl-quinoline (1d, Table 1, entry 4):}

An oven dried pressure vessel containing a magnetic stir bar was charged with ammonium hexafluorophosphate (35 mg, $0.22 \mathrm{mmol}, 1.0$ equiv), $\mathrm{CpRuCl}\left(\mathrm{PPh}_{3}\right)_{2}(16 \mathrm{mg}, 0.022 \mathrm{mmol}, 0.10$ equiv) and SPhos ( $9 \mathrm{mg}, 0.022 \mathrm{mmol}, 0.10$ equiv) under a nitrogen atmosphere in a glove-box and the flask sealed and brought out of the glove-box. Imine 3d $(75 \mathrm{mg}, 0.22 \mathrm{mmol}, 1$ equiv) and toluene $(1.1 \mathrm{~mL})$ were subsequently added via syringe. The flask was flushed with argon, sealed, stirred, and placed in an oil bath at $105^{\circ} \mathrm{C}$. After $19 \mathrm{~h}$, the reaction vessel was allowed to cool to ambient temperature and the mixture was transferred to a recovery flask with a $10-\mathrm{mL}$ portion of dichloromethane. This solution was concentrated under reduced pressure and the residue was purified by flash column chromatography on silica gel (20\% EtoAc/Hexanes) to afford the quinoline 1d as a pale yellow solid (53 mg, 89\%).

${ }^{1} \mathrm{H}$ NMR $\left(500 \mathrm{MHz}, \mathrm{CDCl}_{3}, 20{ }^{\circ} \mathrm{C}\right) \delta:$

${ }^{13} \mathrm{C} \mathrm{NMR}\left(125 \mathrm{MHz}, \mathrm{CDCl}_{3}, 20^{\circ} \mathrm{C}\right) \delta$ :

FTIR (neat) $\mathrm{cm}^{-1}$ :

HRMS (EI):

TLC (20\% EtOAc-hexanes), Rf: $8.46(\mathrm{~s}, 1 \mathrm{H}, \operatorname{ArH}), 8.30(\mathrm{~d}, 1 \mathrm{H}, J=8.2 \mathrm{~Hz}, \operatorname{ArH}), 8.22$ $8.18(\mathrm{~m}, 2 \mathrm{H}, \operatorname{ArH}(o-\mathrm{C}=\mathrm{N})), 8.03(\mathrm{~d}, 1 \mathrm{H}, J=8.6 \mathrm{~Hz}$, $\operatorname{ArH}), 7.97(\mathrm{~d}, 1 \mathrm{H}, J=8.6 \mathrm{~Hz}, \operatorname{ArH}), 7.72(\mathrm{dd}, 1 \mathrm{H}, J=$ 8.5, 1.8 Hz, ArH), 7.59-7.54 (m, 2H, ArH), 7.54-7.50 (m, 1H, ArH).

$158.9,147.5,139.1,136.8,131.7$ (q, $J=32.6 \mathrm{~Hz}$ ), 130.1, $129.2,128.9,127.8$ (q, $J=4.6 \mathrm{~Hz}), 127.5,125.3,123.2$, $122.1(\mathrm{q}, J=2.9 \mathrm{~Hz}), 121.0$.

$3067(w), 3037(w), 2917(w), 1603$ (s), $1316($ s).

calcd for $\mathrm{C}_{16} \mathrm{H}_{10} \mathrm{~F}_{3} \mathrm{~N}[\mathrm{M}]^{+}:$273.0760, found: 273.0750 .

0.46 (UV, CAM). 


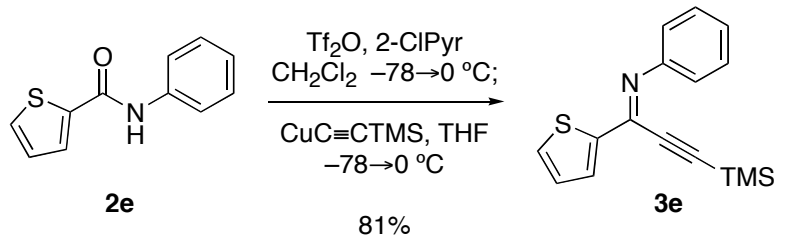

N-Phenyl-2-(thiophen-2-yl)-4-trimethylsilyl-1-azabut-1-en-3-yne (3e, Table 1, entry 5):

Trifluoromethanesulfonic anhydride $(195 \mu \mathrm{L}, 1.18 \mathrm{mmol}, 1.20$ equiv) was added via syringe over $1 \mathrm{~min}$ to a stirred mixture of amide $2 \mathrm{e}(200 \mathrm{mg}, 0.98 \mathrm{mmol}, 1 \mathrm{equiv})$ and 2-chloropyridine (372 $\mu \mathrm{L}, 3.94 \mathrm{mmol}, 4.00$ equiv) in $\mathrm{CH}_{2} \mathrm{Cl}_{2}(2.0 \mathrm{~mL})$ at $-78{ }^{\circ} \mathrm{C}$. After $5 \mathrm{~min}$, the reaction mixture was warmed to $0{ }^{\circ} \mathrm{C}$. After $20 \mathrm{~min}$, the solution was cooled to $-78^{\circ} \mathrm{C}$ and a freshly prepared solution of copper (I) (trimethylysilyl)-acetylide (428 mg, $2.66 \mathrm{mmol}, 2.70$ equiv) in THF $(5.0 \mathrm{~mL})$ at $0{ }^{\circ} \mathrm{C}$ was added via cannula. The reaction mixture was kept at $-78{ }^{\circ} \mathrm{C}$ for $5 \mathrm{~min}$ and then warmed to $0{ }^{\circ} \mathrm{C}$. After $10 \mathrm{~min}$, the crude reaction mixture was filtered through celite $(2 \mathrm{~cm}$ diam. $\times 3 \mathrm{~cm} \mathrm{ht}$.) and the filtrate was concentrated under reduced pressure. The residue was purified by flash column chromatography on silica gel $(100 \%$ hexanes $\rightarrow 7.5 \%$ EtOAc/hexanes) to afford the alkynyl imine $3 \mathbf{e}$ as a pale yellow oil (227 $\mathrm{mg}, 81 \%)$.

${ }^{1} \mathrm{H}$ NMR (500 MHz, $\left.\mathrm{CDCl}_{3}, 20{ }^{\circ} \mathrm{C}\right) \delta: \quad 7.73(\mathrm{dd}, 1 \mathrm{H}, J=3.7,1.2 \mathrm{~Hz}, \mathrm{SCHCHCHCC}=\mathrm{N}), 7.48$ $(\mathrm{dd}, 1 \mathrm{H}, J=5.0,0.9 \mathrm{~Hz}, \mathrm{SCHCHCHCC}=\mathrm{N}), 7.38-7.33$ (m, 2H, ArH), 7.20-7.14 (m, 3H, ArH), 7.12 (dd, 1H, $J$ $=4.6,3.7 \mathrm{~Hz}, \mathrm{SCHCHCHCC}=\mathrm{N}), 0.16(\mathrm{~s}, 9 \mathrm{H}$, $\left.\mathrm{Si}\left(\mathrm{CH}_{3}\right)_{3}\right)$.

${ }^{13} \mathrm{C}$ NMR $\left(125 \mathrm{MHz}, \mathrm{CDCl}_{3}, 20{ }^{\circ} \mathrm{C}\right) \delta: \quad 150.5,144.7,144.0,131.6,130.6,128.5,127.7,125.4$, $121.6,103.7,96.7,-0.5$.

FTIR (neat) $\mathrm{cm}^{-1}$ :

HRMS (ESI):

TLC (20\% EtOAc/hexanes), Rf: $3078(\mathrm{~m}), 2960$ (s), 1563 (s, C=N), 1425 (s), 1252 (s).

calcd for $\mathrm{C}_{16} \mathrm{H}_{18} \mathrm{NSSi}[\mathrm{M}+\mathrm{H}]^{+}$: 284.0924, found: 284.0937.

0.70 (UV, CAM). 


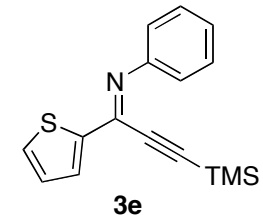

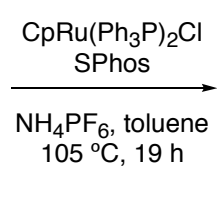

$75 \%$

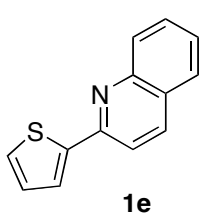

1e

\section{2-Thiophen-2-ylquinoline (1e, Table 1, entry 5):}

An oven dried pressure vessel containing a magnetic stir bar was charged with ammonium hexafluorophosphate (58 mg, $0.35 \mathrm{mmol}, 1.0$ equiv), $\mathrm{CpRu}\left(\mathrm{PPh}_{3}\right)_{2} \mathrm{Cl}(26 \mathrm{mg}, 0.035 \mathrm{mmol}, 0.10$ equiv) and SPhos (15 mg, $0.035 \mathrm{mmol}, 0.10$ equiv) under a nitrogen atmosphere in a glove-box and the flask sealed and brought out of the glove-box. Imine $3 \mathbf{e}(100 \mathrm{mg}, 0.35 \mathrm{mmol}, 1$ equiv) and toluene $(1.8 \mathrm{~mL})$ were subsequently added via syringe. The flask was flushed with argon, sealed, stirred, and placed in an oil bath at $105^{\circ} \mathrm{C}$. After $19 \mathrm{~h}$, the reaction vessel was allowed to cool to ambient temperature and the mixture was transferred to a recovery flask with a $10-\mathrm{mL}$ portion of dichloromethane. This solution was concentrated under reduced pressure and the residue was purified by flash column chromatography on silica gel (20\% EtoAc/Hexanes) to afford the quinoline $1 \mathrm{e}^{13}$ as a pale yellow solid (56 mg, 75\%).

${ }^{1} \mathrm{H}$ NMR $\left(500 \mathrm{MHz}, \mathrm{CDCl}_{3}, 20^{\circ} \mathrm{C}\right)$ :

${ }^{13} \mathrm{C} \mathrm{NMR}\left(125 \mathrm{MHz}, \mathrm{CDCl}_{3}, 20^{\circ} \mathrm{C}\right)$ :

FTIR (neat):

HRMS (ESI):

TLC (20\% EtOAc-hexanes), Rf: $8.16(\mathrm{~d}, 1 \mathrm{H}, J=8.9 \mathrm{~Hz}, \operatorname{ArH}), 8.10(\mathrm{~d}, 1 \mathrm{H}, J=9.2 \mathrm{~Hz}$, $\operatorname{ArH}), 7.82(\mathrm{~d}, 1 \mathrm{H}, J=8.6 \mathrm{~Hz}, \operatorname{ArH}), 7.79(\mathrm{~d}, 1 \mathrm{H}, J=$ $7.9 \mathrm{~Hz}, \operatorname{ArH}(\mathrm{SCHCHCHCC}=\mathrm{N})), 7.75(\mathrm{dd}, 1 \mathrm{H}, J=3.7$, $0.9 \mathrm{~Hz}, \operatorname{ArH}(\mathrm{SCHCHCHCC}=\mathrm{N})), 7.71(\mathrm{ddd}, 1 \mathrm{H}, J=$ 8.5, 7.0, 1.5 Hz, ArH), 7.52-7.47 (m, 2H, ArH), 7.18 $(\mathrm{dd}, 1 \mathrm{H}, J=4.9,3.7 \mathrm{~Hz}, \operatorname{ArH}(\mathrm{SCHCHCHC}=\mathrm{N}))$.

$152.5,148.3,145.6,136.8,130.0,129.4,128.8,128.3$, $127.7,127.3,126.3,126.0,117.8$.

$3079(\mathrm{w}), 3042(\mathrm{w}), 1962(\mathrm{w}), 1617(\mathrm{~m}), 1597(\mathrm{~s}), 1561$ (m), $1502(\mathrm{~s})$.

calcd for $\mathrm{C}_{13} \mathrm{H}_{10} \mathrm{NS}[\mathrm{M}+\mathrm{H}]^{+}:$212.0528, found: 212.0533 .

$0.40\left(\mathrm{UV}, \mathrm{KMnO}_{4}\right)$.

\footnotetext{
${ }^{13}$ Fürstner, A.; Leitner, A.; Méndez, M.; Krause, H. J. Am. Chem. Soc. 2002, 124, 13856-13863.
} 


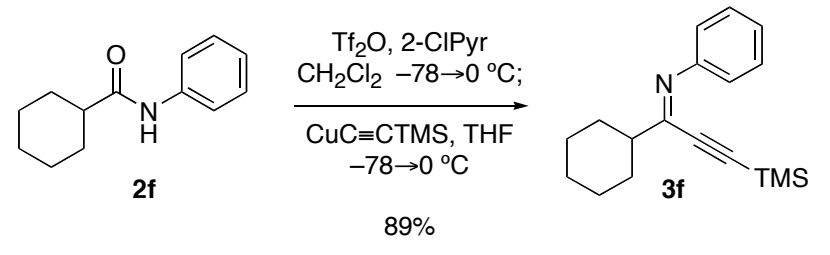

\section{N-Phenyl-2-cyclohexyl-4-trimethylsilyl-1-azabut-1-en-3-yne (3f, Table 1, entry 6):}

Trifluoromethanesulfonic anhydride $(98 \mu \mathrm{L}, 0.59 \mathrm{mmol}, 1.20$ equiv) was added via syringe over $1 \mathrm{~min}$ to a stirred mixture of amide $\mathbf{2 f}(100 \mathrm{mg}, 0.49 \mathrm{mmol}, 1$ equiv) and 2-chloropyridine (186 $\mu \mathrm{L}, 1.97 \mathrm{mmol}, 4.00$ equiv) in $\mathrm{CH}_{2} \mathrm{Cl}_{2}(1.0 \mathrm{~mL})$ at $-78{ }^{\circ} \mathrm{C}$. After $5 \mathrm{~min}$, the reaction mixture was warmed to $0{ }^{\circ} \mathrm{C}$. After $20 \mathrm{~min}$, the solution was cooled to $-78{ }^{\circ} \mathrm{C}$ and a freshly prepared solution of copper (I) (trimethylysilyl)-acetylide (214 mg, $1.33 \mathrm{mmol}, 2.70$ equiv) in THF $(3.0 \mathrm{~mL})$ at $0{ }^{\circ} \mathrm{C}$ was added via cannula. The reaction mixture was kept at $-78{ }^{\circ} \mathrm{C}$ for $5 \mathrm{~min}$ and then warmed to $0{ }^{\circ} \mathrm{C}$. After $10 \mathrm{~min}$, the crude reaction mixture was filtered through celite $(2 \mathrm{~cm}$ diam. $\times 3 \mathrm{~cm} \mathrm{ht}$.) and the filtrate was concentrated under reduced pressure. The residue was purified by flash column chromatography on neutralized silica gel $\left(1 \% \mathrm{Et}_{3} \mathrm{~N} /\right.$ hexanes $)$ to afford the alkynyl imine $\mathbf{3 f}$ as a pale yellow oil (123 mg, 89\%).

${ }^{1} \mathrm{H}$ NMR $\left(500 \mathrm{MHz}, \mathrm{CDCl}_{3}, 20^{\circ} \mathrm{C}\right)$ :

${ }^{13} \mathrm{C} \mathrm{NMR}\left(125 \mathrm{MHz}, \mathrm{CDCl}_{3}, 20^{\circ} \mathrm{C}\right)$ :

FTIR (neat):

HRMS (ESI):

TLC (20\% EtOAc-hexanes), Rf:
7.32-7.28 (m, 2H, ArH), 7.12-7.07 (m, 1H, ArH), 6.96$6.93(\mathrm{~m}, 2 \mathrm{H}, \mathrm{ArH}), 2.47(\mathrm{tt}, 1 \mathrm{H}, J=23.2,6.9 \mathrm{~Hz}$, $\left.\left(\mathrm{CH}_{2}\right)_{2} \mathrm{CHC}=\mathrm{N}\right), 2.01$ (br dd, $2 \mathrm{H}, J=12.6,2.0 \mathrm{~Hz}$, $\left.{ }^{c} \mathrm{C}_{6} \mathbf{H}_{11}\right), 1.86\left(\mathrm{dt}, 2 \mathrm{H}, J=13.1,3.4 \mathrm{~Hz},{ }^{c} \mathrm{C}_{6} \mathbf{H}_{11}\right), 1.76-$ $1.69\left(\mathrm{~m}, 1 \mathrm{H},{ }^{c} \mathrm{C}_{6} \mathbf{H}_{11}\right), 1.52(\mathrm{qd}, 2 \mathrm{H}, J=12.5,3.4 \mathrm{~Hz}$, $\left.{ }^{c} \mathrm{C}_{6} \mathbf{H}_{11}\right), 1.36\left(\mathrm{qt}, 2 \mathrm{H}, J=12.5,3.2 \mathrm{~Hz},{ }^{c} \mathrm{C}_{6} \mathbf{H}_{11}\right), 1.26$ (qt, $\left.1 \mathrm{H}, J=12.5,3.2 \mathrm{~Hz},{ }^{c} \mathrm{C}_{6} \mathbf{H}_{11}\right), 0.06\left(\mathrm{~s}, 9 \mathrm{H}, \mathrm{Si}\left(\mathrm{CH}_{3}\right)_{3}\right)$.

$159.6,151.7,128.4,124.5,120.6,104.0,98.2,48.3,30.3$, $26.1,26.0,-0.5$.

3049 (w), 2933 (s), 2856 (m), 1660 (w), 1594 (s), 1484 (m).

calcd for $\mathrm{C}_{18} \mathrm{H}_{26} \mathrm{NSi}[\mathrm{M}+\mathrm{H}]^{+}$: 284.1829, found: 284.1836.

$0.67\left(\mathrm{UV}, \mathrm{KMnO}_{4}\right)$. 

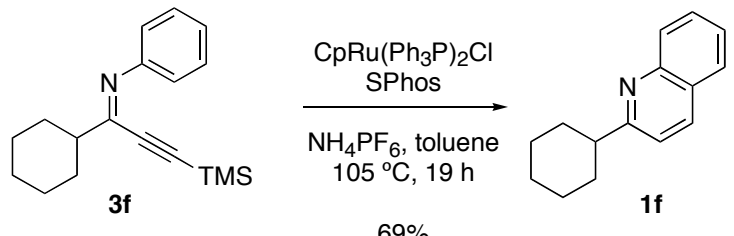

\section{2-Cyclohexyl-quinoline (1f, Table 1, entry 6):}

An oven dried pressure vessel containing a magnetic stir bar was charged with ammonium hexafluorophosphate (40 mg, $0.25 \mathrm{mmol}, 1.0$ equiv), $\mathrm{CpRu}\left(\mathrm{PPh}_{3}\right)_{2} \mathrm{Cl}$ (18 mg, $0.025 \mathrm{mmol}, 0.10$ equiv) and SPhos (10 mg, $0.025 \mathrm{mmol}, 0.10$ equiv) under a nitrogen atmosphere in a glove-box and the flask sealed and brought out of the glove-box. Imine $\mathbf{3 f}(70 \mathrm{mg}, 0.25 \mathrm{mmol}, 1$ equiv) and toluene (1.2 $\mathrm{mL})$ were subsequently added via syringe. The flask was flushed with argon, sealed, stirred, and placed in an oil bath at $105^{\circ} \mathrm{C}$. After $19 \mathrm{~h}$, the reaction vessel was allowed to cool to ambient temperature and the mixture was transferred to a recovery flask with a $10-\mathrm{mL}$ portion of dichloromethane. This solution was concentrated under reduced pressure and the residue was purified by flash column chromatography on silica gel (10\% EtoAc/Hexanes) to afford the quinoline $\mathbf{1 f}^{14}$ as a pale yellow solid (36 mg, 69\%).

${ }^{1} \mathrm{H}$ NMR $\left(500 \mathrm{MHz}, \mathrm{CDCl}_{3}, 20^{\circ} \mathrm{C}\right)$ :

${ }^{13} \mathrm{C} \mathrm{NMR}\left(125 \mathrm{MHz}, \mathrm{CDCl}_{3}, 20^{\circ} \mathrm{C}\right)$ :

FTIR (neat):

HRMS (EI):

TLC (20\% EtOAc-hexanes), $R f$ : $8.09(\mathrm{~d}, 1 \mathrm{H}, J=8.5 \mathrm{~Hz}, \operatorname{ArH}), 8.06(\mathrm{~d}, 1 \mathrm{H}, J=8.5 \mathrm{~Hz}$, ArH), 7.78 (d, 1H, $J=8.2 \mathrm{~Hz}, \operatorname{ArH}), 7.69$ (ddd, 1H, $J=$ 8.5, 7.0, $1.5 \mathrm{~Hz}, \mathrm{ArH}), 7.49$ (ddd, $1 \mathrm{H}, J=7.9,7.0,1.2$ $\mathrm{Hz}, \operatorname{ArH}), 7.35$ (d, 1H, $J=8.6 \mathrm{~Hz}, \operatorname{ArH}), 2.94(\mathrm{tt}, 1 \mathrm{H}, J$ $\left.=12.1,3.4 \mathrm{~Hz},\left(\mathrm{CH}_{2}\right)_{2} \mathrm{CHCN}\right), 2.08-2.00(\mathrm{~m}, 2 \mathrm{H}$, $\left.{ }^{c} \mathrm{C}_{6} \mathbf{H}_{11}\right), 1.98-1.87\left(\mathrm{~m}, 2 \mathrm{H},{ }^{c} \mathrm{C}_{6} \mathbf{H}_{11}\right), 1.86-1.76(\mathrm{~m}, 1 \mathrm{H}$, $\left.{ }^{c} \mathrm{C}_{6} \mathbf{H}_{11}\right), 1.64\left(\mathrm{qd}, 2 \mathrm{H}, J=12.5,3.1 \mathrm{~Hz},{ }^{c} \mathrm{C}_{6} \mathbf{H}_{11}\right), 1.49$ (qt, $2 \mathrm{H}, J=13.1,3.4 \mathrm{~Hz},{ }^{c} \mathrm{C}_{6} \mathbf{H}_{11}$ ), 1.35 (qt, $1 \mathrm{H}, J=12.8,3.4$ $\left.\mathrm{Hz},{ }^{c} \mathrm{C}_{6} \mathbf{H}_{11}\right)$.

$167.0,148.0,136.5,129.4,129.1,127.6,127.2,125.8$, $119.8,47.9,33.0,26.7,26.3$.

$3058(w), 2924(\mathrm{~s}), 2850(\mathrm{~m}), 1720(\mathrm{w}), 1619(\mathrm{w}), 1601$ (m), $1502(\mathrm{~m})$.

calcd for $\mathrm{C}_{15} \mathrm{H}_{17} \mathrm{~N}[\mathrm{M}]^{+}:$211.1356, found: 211.1358 .

$0.50\left(\mathrm{UV}, \mathrm{KMnO}_{4}\right)$.

\footnotetext{
${ }^{14}$ For a prior synthesis (characterization data not listed), see: Ishikura, M.; Oda, I,; Kamada, M.; Terashima, M. Synth. Comm. 1987, 17, 959-967.
} 


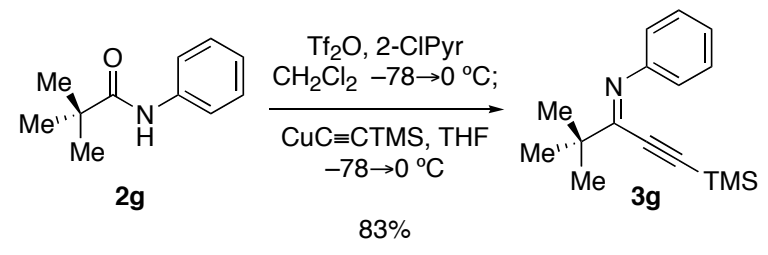

\section{N-Phenyl-2-(tert-butyl)-4-trimethylsilyl-1-azabut-1-en-3-yne (3g, Table 1, entry 7):}

Trifluoromethanesulfonic anhydride $(220 \mu \mathrm{L}, 1.33 \mathrm{mmol}, 1.20$ equiv) was added via syringe over $1 \mathrm{~min}$ to a stirred mixture of amide $2 \mathrm{~g}$ (197 $\mathrm{mg}, 1.11 \mathrm{mmol}, 1$ equiv) and 2-chloropyridine (420 $\mu \mathrm{L}, 4.44 \mathrm{mmol}, 4.00$ equiv) in $\mathrm{CH}_{2} \mathrm{Cl}_{2}(2.2 \mathrm{~mL})$ at $-78{ }^{\circ} \mathrm{C}$. After $5 \mathrm{~min}$, the reaction mixture was warmed to $0{ }^{\circ} \mathrm{C}$. After $20 \mathrm{~min}$, the solution was cooled to $-78^{\circ} \mathrm{C}$ and a freshly prepared solution of copper (I) (trimethylysilyl)-acetylide (482 mg, $3.00 \mathrm{mmol}, 2.70$ equiv) in THF $(5.0 \mathrm{~mL})$ at $0{ }^{\circ} \mathrm{C}$ was added via cannula. The reaction mixture was kept at $-78{ }^{\circ} \mathrm{C}$ for $5 \mathrm{~min}$ and then warmed to $0{ }^{\circ} \mathrm{C}$. After $10 \mathrm{~min}$, the crude reaction mixture was filtered through celite $(2 \mathrm{~cm}$ diam. $\times 3 \mathrm{~cm} \mathrm{ht}$.) and the filtrate was concentrated under reduced pressure. The residue was purified by flash column chromatography on silica gel $(100 \%$ hexanes $\rightarrow 7.5 \%$ EtOAc/hexanes) to afford the alkynyl imine $\mathbf{3 g}$ as a pale yellow oil (236 $\mathrm{mg}, 83 \%)$.

${ }^{1} \mathrm{H}$ NMR $\left(500 \mathrm{MHz}, \mathrm{CDCl}_{3}, 20{ }^{\circ} \mathrm{C}\right) \delta: \quad 7.32-7.28(\mathrm{~m}, 2 \mathrm{H}, \mathrm{ArH}), 7.11-7.06(\mathrm{~m}, 1 \mathrm{H}, \mathrm{ArH}), 6.92-$ $6.88(\mathrm{~m}, 2 \mathrm{H}, \mathrm{ArH}), 1.31\left(\mathrm{~s}, 9 \mathrm{H}, \mathrm{C}\left(\mathrm{CH}_{3}\right)_{3}\right), 0.50(\mathrm{~s}, 9 \mathrm{H}$, $\left.\mathrm{Si}\left(\mathrm{CH}_{3}\right)_{3}\right)$.

${ }^{13} \mathrm{C} \mathrm{NMR}\left(125 \mathrm{MHz}, \mathrm{CDCl}_{3}, 20{ }^{\circ} \mathrm{C}\right) \delta: \quad 162.9,152.2,128.5,124.2,120.3,104.7,97.3,39.8,28.0$, -0.5 .

FTIR (neat) $\mathrm{cm}^{-1}$ :

$3080(w), 2967$ (s), 2868 (m), 1931 (w), 1593 (s), 1477

(s).

HRMS (ESI):

calcd for $\mathrm{C}_{16} \mathrm{H}_{24} \mathrm{NSi}[\mathrm{M}+\mathrm{H}]^{+}:$258.1673, found: 258.1675.

TLC (20\% EtOAc-hexanes), Rf: 0.69 (UV, CAM). 

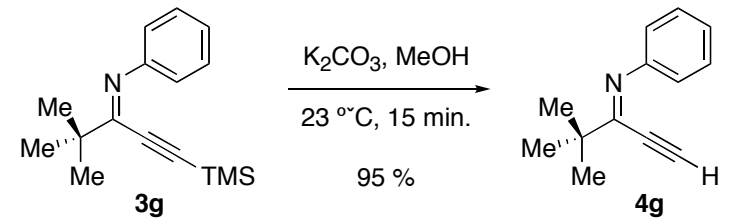

(1-tert-Butyl-prop-2-ynylidene)-phenyl-amine (4g, Table 1, entry 7):

Anhydrous potassium carbonate $(41 \mathrm{mg}, 0.30 \mathrm{mmol}, 0.2$ equiv) was added to a solution of imine $3 \mathrm{~g}$ (380 mg, $1.48 \mathrm{mmol}, 1$ equiv) in methanol $(5.0 \mathrm{~mL})$ and stirred at $23{ }^{\circ} \mathrm{C}$. After $25 \mathrm{~min}$, the volatiles were removed under reduced pressure and the residue was purified by flash column chromatography on silica gel $(7.5 \%$ EtOAc/hexanes) to afford the alkynyl imine $\mathbf{4 g}$ as a yellow solid (259 mg, 95\%).

${ }^{1} \mathrm{H}$ NMR $\left(500 \mathrm{MHz}, \mathrm{CDCl}_{3}, 20^{\circ} \mathrm{C}\right)$ :

7.32-7.28 (m, 2H, ArH), $7.12(\mathrm{tt}, 1 \mathrm{H}, J=7.3,2.3 \mathrm{~Hz}$, ArH), 6.92-6.88 (m, 2H, ArH), 3.14 (s, 1H, C $\equiv \mathrm{CH})$, $1.33\left(\mathrm{~s}, 9 \mathrm{H}, \mathrm{C}\left(\mathrm{CH}_{3}\right)_{3}\right)$.

${ }^{13} \mathrm{C} \mathrm{NMR}\left(125 \mathrm{MHz}, \mathrm{CDCl}_{3}, 20^{\circ} \mathrm{C}\right)$ :

$161.9,151.8,128.7,124.4,119.9,85.4,76.2,40.2,27.8$.

FTIR (neat):

2962 (s), 2925 (s), 1734 (m), 1717 (m), 1684 (s), 1653

(m).

HRMS (EI):

calcd for $\mathrm{C}_{13} \mathrm{H}_{15} \mathrm{~N}[\mathrm{M}]^{+}:$185.1199,

found: 185.1193 .

TLC (20\% EtOAc-hexanes), Rf:

$0.66\left(\mathrm{UV}, \mathrm{KMnO}_{4}\right)$. 

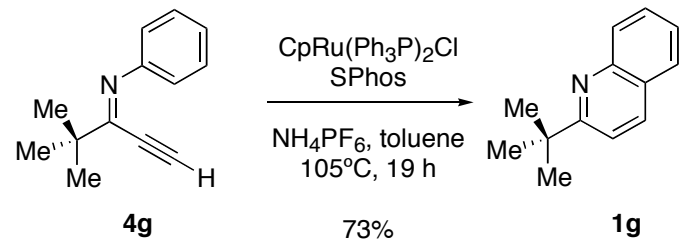

\section{2-tert-Butyl-quinoline (1g, Table 1, entry 7):}

An oven dried pressure vessel containing a magnetic stir bar was charged with ammonium hexafluorophosphate (62 mg, $0.38 \mathrm{mmol}, 1.0$ equiv), $\mathrm{CpRu}\left(\mathrm{PPh}_{3}\right)_{2} \mathrm{Cl}$ ( $28 \mathrm{mg}, 0.038 \mathrm{mmol}, 0.10$ equiv) and SPhos (16 mg, $0.038 \mathrm{mmol}, 0.10$ equiv) under a nitrogen atmosphere in a glove-box and the flask sealed and brought out of the glove-box. Imine $4 \mathrm{~g}(70 \mathrm{mg}, 0.38 \mathrm{mmol}, 1$ equiv) and toluene $(1.9 \mathrm{~mL})$ were subsequently added via syringe. The flask was flushed with argon, sealed, stirred, and placed in an oil bath at $105^{\circ} \mathrm{C}$. After $19 \mathrm{~h}$, the reaction vessel was allowed to cool to ambient temperature and the mixture was transferred to a recovery flask with a $10-\mathrm{mL}$ portion of dichloromethane. This solution was concentrated under reduced pressure and the residue was purified by flash column chromatography on silica gel (7.5\% EtoAc/Hexanes) to afford the quinoline $\mathbf{1 g}$ as a pale yellow solid (51 mg, 73\%).

${ }^{1} \mathrm{H}$ NMR $\left(500 \mathrm{MHz}, \mathrm{CDCl}_{3}, 20^{\circ} \mathrm{C}\right)$ :

${ }^{13} \mathrm{C} \mathrm{NMR}\left(125 \mathrm{MHz}, \mathrm{CDCl}_{3}, 20^{\circ} \mathrm{C}\right)$ :

FTIR (neat):

HRMS (ESI):

TLC (20\% EtOAc-hexanes), Rf:
8.09 (d, 1H, $J=8.2 \mathrm{~Hz}, \operatorname{ArH}), 8.07$ (d, $1 \mathrm{H}, J=8.5 \mathrm{~Hz}$, ArH), 7.78 (dd, 1H, J=7.9, 1.2 Hz, ArH), 7.65 (ddd, $1 \mathrm{H}, J=8.5,7.0,1.5 \mathrm{~Hz}, \operatorname{ArH}), 7.54(\mathrm{~d}, 1 \mathrm{H}, J=8.9 \mathrm{~Hz}$, ArH), 7.48 (ddd, 1H, $J=8.1,6.7,0.9 \mathrm{~Hz}, \operatorname{ArH}), 1.49$ (s, $\left.9 \mathrm{H}, \mathrm{C}\left(\mathrm{CH}_{3}\right)_{3}\right)$.

$147.6,136.1,129.6,129.2,127.4,126.6,125.8,118.5$, $100.0,38.3,30.4$.

3061 (w), 2960 (s), 2868 (m), 1619 (m), 1601 (s), 1565 (w), 1503 (s).

calcd for $\mathrm{C}_{13} \mathrm{H}_{16} \mathrm{~N}[\mathrm{M}+\mathrm{H}]^{+}$: 186.1277 , found: 186.1283 .

$0.46\left(\mathrm{UV}, \mathrm{KMnO}_{4}\right)$ 


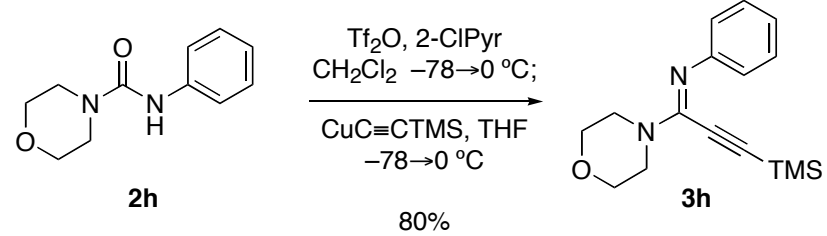

\section{N-Phenyl-2-(morpholin-4-yl)-4-trimethylsilyl-1-azabut-1-en-3-yne (3h, Table 1, entry 8):}

Trifluoromethanesulfonic anhydride $(144 \mu \mathrm{L}, 0.87 \mathrm{mmol}, 1.20$ equiv) was added via syringe over $1 \mathrm{~min}$ to a stirred mixture of amide $\mathbf{2 h}(150 \mathrm{mg}, 0.73 \mathrm{mmol}, 1$ equiv) and 2-chloropyridine (275 $\mu \mathrm{L}, 2.91 \mathrm{mmol}, 4.00$ equiv) in $\mathrm{CH}_{2} \mathrm{Cl}_{2}(1.5 \mathrm{~mL})$ at $-78{ }^{\circ} \mathrm{C}$. After $5 \mathrm{~min}$, the reaction mixture was warmed to $0{ }^{\circ} \mathrm{C}$. After $20 \mathrm{~min}$, the solution was cooled to $-78{ }^{\circ} \mathrm{C}$ and Hünig's base $(190 \mu \mathrm{L}, 1.09$ mmol, 1.50 equiv) was added via syringe followed by a freshly prepared solution of copper (I) (trimethylysilyl)-acetylide (316 mg, $1.96 \mathrm{mmol}, 2.70$ equiv) in THF $(3.0 \mathrm{~mL})$ at $0{ }^{\circ} \mathrm{C}$ via cannula. The reaction mixture was kept at $-78^{\circ} \mathrm{C}$ for $5 \mathrm{~min}$ and then warmed to $0{ }^{\circ} \mathrm{C}$. After $10 \mathrm{~min}$, the crude reaction mixture was filtered through celite $(2 \mathrm{~cm}$ diam. $\times 3 \mathrm{~cm} \mathrm{ht}$.) and the filtrate was concentrated under reduced pressure. The residue was purified by flash column chromatography on neutralized silica gel (50\% EtOAc/hexanes) to afford the alkynyl imidate $\mathbf{3 h}$ as a burgandy colored oil (166 $\mathrm{mg}$, $80 \%)$.

${ }^{1} \mathrm{H} \mathrm{NMR}\left(500 \mathrm{MHz}, \mathrm{CDCl}_{3}, 20^{\circ} \mathrm{C}\right)$ :

${ }^{13} \mathrm{C} \mathrm{NMR}\left(125 \mathrm{MHz}, \mathrm{CDCl}_{3}, 20^{\circ} \mathrm{C}\right)$ :

FTIR (neat):

HRMS (ESI):

TLC (50\% EtOAc-hexanes), Rf:
7.27-7.23 (m, 2H, ArH), $7.01(\mathrm{tt}, 1 \mathrm{H}, J=7.3,1.1 \mathrm{~Hz}$, ArH), 6.92-6.88 (m, 2H, ArH), 3.79-3.75 (m, 4H, $\mathrm{OCH}_{2} \mathrm{CH}_{2} \mathrm{~N}$ ), 3.69-3.66 (m, 4H, $\left.\mathrm{OCH}_{2} \mathrm{CH}_{2} \mathrm{~N}\right), 0.04$ (s, $\left.9 \mathrm{H}, \mathrm{Si}\left(\mathrm{CH}_{3}\right)_{3}\right)$.

$151.2,144.2,128.5,122.9,122.3,104.2,93.2,66.8,45.9$, -0.7 .

2967 (m), 2924 (w), 2861 (m), 1577 (s, C=N), 1420 (m) 1247 (s).

calcd for $\mathrm{C}_{16} \mathrm{H}_{23} \mathrm{~N}_{2} \mathrm{OSi}[\mathrm{M}+\mathrm{H}]^{+}$: 287.1574, found: 287.1580 .

$0.70\left(\mathrm{UV}, \mathrm{KMnO}_{4}\right)$. 

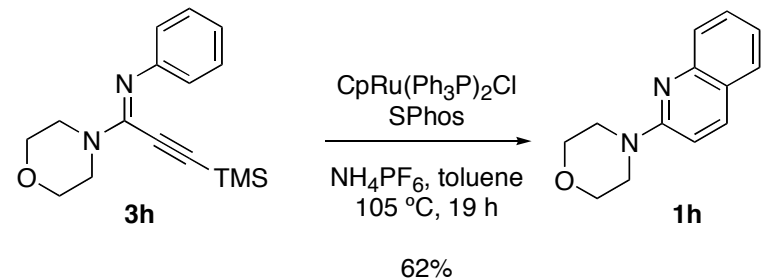

$62 \%$

\section{2-Morpholin-4-yl-quinoline (1h, Table 1, entry 8):}

An oven dried pressure vessel containing a magnetic stir bar was charged with ammonium hexafluorophosphate (43 mg, $0.26 \mathrm{mmol}, 1.0$ equiv), $\mathrm{CpRu}\left(\mathrm{PPh}_{3}\right)_{2} \mathrm{Cl}$ (19 mg, $0.026 \mathrm{mmol}, 0.10$ equiv) and SPhos (11 mg, $0.026 \mathrm{mmol}, 0.10$ equiv) under a nitrogen atmosphere in a glove-box and the flask sealed and brought out of the glove-box. Imine $\mathbf{3 h}$ ( $75 \mathrm{mg}, 0.26 \mathrm{mmol}, 1$ equiv) and toluene (1.3 $\mathrm{mL})$ were subsequently added via syringe. The flask was flushed with argon, sealed, stirred, and placed in an oil bath at $105^{\circ} \mathrm{C}$. After $19 \mathrm{~h}$, the reaction vessel was allowed to cool to ambient temperature and the mixture was transferred to a recovery flask with a $10-\mathrm{mL}$ portion of dichloromethane. This solution was concentrated under reduced pressure and the residue was purified by flash column chromatography on silica gel (20\% EtoAc/Hexanes) to afford the quinoline $\mathbf{1 h}$ as a pale yellow solid (35 mg, 62\%).

${ }^{1} \mathrm{H}$ NMR $\left(500 \mathrm{MHz}, \mathrm{CDCl}_{3}, 20^{\circ} \mathrm{C}\right)$ :

${ }^{13} \mathrm{C}$ NMR $\left(125 \mathrm{MHz}, \mathrm{CDCl}_{3}, 20^{\circ} \mathrm{C}\right)$ :

FTIR (neat):

HRMS (ESI):

TLC (20\% EtOAc-hexanes), Rf: $7.95(\mathrm{~d}, 1 \mathrm{H}, J=8.7 \mathrm{~Hz}, \operatorname{ArH}), 7.74(\mathrm{~d}, 1 \mathrm{H}, J=7.6 \mathrm{~Hz}$, ArH), 7.65 (dd, $1 \mathrm{H}, J=7.9,1.5 \mathrm{~Hz}, \operatorname{ArH}), 7.56$ (ddd, $1 \mathrm{H}, J=8.5,7.0,1.5 \mathrm{~Hz}, \mathrm{ArH}), 7.27$ (ddd, $1 \mathrm{H}, J=8.0$, 6.7, $0.9 \mathrm{~Hz}, \operatorname{ArH}), 6.99$ (d, 1H, J=9.2 Hz, ArH), 3.87 (t, $\left.4 \mathrm{H}, J=4.9, \mathrm{OCH}_{2} \mathrm{CH}_{2} \mathrm{~N}\right), 3.73(\mathrm{t}, 4 \mathrm{H}, J=4.9 \mathrm{~Hz}$, $\mathrm{OCH}_{2} \mathrm{CH}_{2} \mathrm{~N}$ ).

$157.6,147.8,137.7,129.8,127.4,126.9,123.4,122.8$, 109.4, 67.0, 45.7 .

3047 (w), 2972 (w), 2917 (w), 2858 (m), 1617 (s), 1605

(m).

calcd for $\mathrm{C}_{13} \mathrm{H}_{14} \mathrm{~N}_{2} \mathrm{O}[\mathrm{M}]^{+}$: 215.1179, found: 215.1178 .

$0.21\left(\mathrm{UV}, \mathrm{KMnO}_{4}\right)$. 

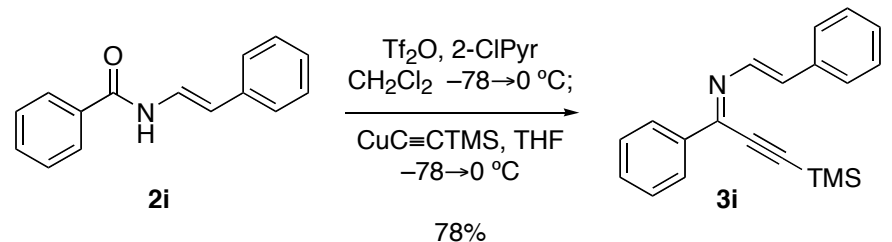

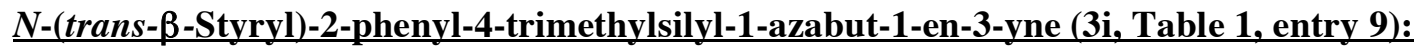

Trifluoromethanesulfonic anhydride $(133 \mu \mathrm{L}, 0.81 \mathrm{mmol}, 1.20$ equiv) was added via syringe over $1 \mathrm{~min}$ to a stirred mixture of amide $2 \mathbf{i}$ ( $150 \mathrm{mg}, 0.67 \mathrm{mmol}, 1$ equiv) and 2-chloropyridine (254 $\mu \mathrm{L}, 2.69 \mathrm{mmol}, 4.00$ equiv) in $\mathrm{CH}_{2} \mathrm{Cl}_{2}(1.3 \mathrm{~mL})$ at $-78^{\circ} \mathrm{C}$. After $5 \mathrm{~min}$, the reaction mixture was warmed to $0{ }^{\circ} \mathrm{C}$. After $20 \mathrm{~min}$, the solution was cooled to $-78^{\circ} \mathrm{C}$ and a freshly prepared solution of copper (I) (trimethylysilyl)-acetylide (292 mg, $1.81 \mathrm{mmol}, 2.70$ equiv) in THF $(5.0 \mathrm{~mL})$ at $0^{\circ} \mathrm{C}$ was added via cannula. The reaction mixture was kept at $-78^{\circ} \mathrm{C}$ for $5 \mathrm{~min}$ and then warmed to $0{ }^{\circ} \mathrm{C}$. After $10 \mathrm{~min}$, the crude reaction mixture was filtered through celite $(2 \mathrm{~cm}$ diam. $\times 3 \mathrm{~cm} \mathrm{ht}$.) and the filtrate was concentrated under reduced pressure. The residue was purified by flash column chromatography on silica gel $(100 \%$ hexanes $\rightarrow 7.5 \%$ EtOAc/hexanes) to afford the alkynyl imine $3 \mathbf{i}$ as a pale yellow solid (159 mg, 78\%).

${ }^{1} \mathrm{H} \mathrm{NMR}\left(500 \mathrm{MHz}, \mathrm{CDCl}_{3}, 20^{\circ} \mathrm{C}\right)$ :

$8.34(\mathrm{~d}, 1 \mathrm{H}, J=13.1 \mathrm{~Hz}, \mathrm{NCHCHC}), 8.18-8.13(\mathrm{~m}, 2 \mathrm{H}$, ArH), 7.56 (d, 2H, J=7.3 Hz, ArH), 7.48-7.42 (m, 3H, $\operatorname{ArH}), 7.39$ (t, 2H, $J=7.6 \mathrm{~Hz}, \operatorname{ArH}), 7.31$ (tt, 1H, $J=7.3$, $1.2 \mathrm{~Hz}, \operatorname{ArH}), 7.16(\mathrm{~d}, 1 \mathrm{H}, J=13.4 \mathrm{~Hz}, \mathrm{NCHCHC})$, 0.39 (s, 9H, $\left.\mathrm{Si}\left(\mathrm{CH}_{3}\right)_{3}\right)$.

${ }^{13} \mathrm{C}$ NMR $\left(125 \mathrm{MHz}, \mathrm{CDCl}_{3}, 20^{\circ} \mathrm{C}\right) \delta:$ $148.0,139.7,137.1,136.7,133.8,131.0,129.0,128.6$, $128.5,128.0,127.4,108.7,96.8,0.0$.

FTIR $\left(\mathrm{CDCl}_{3}\right) \mathrm{cm}^{-1}$ : $3155(\mathrm{~m}), 3062(\mathrm{~m}), 3028(\mathrm{~m}), 2963(\mathrm{~m}), 2902(\mathrm{~m})$, 1815 (m), 1794 (m), 1643 (w), 1622 (m), 1490 (s).

HRMS (ESI):

calcd for $\mathrm{C}_{20} \mathrm{H}_{22} \mathrm{NSi}[\mathrm{M}+\mathrm{H}]^{+}:$304.1516, found: 304.1526 .

TLC (20\% EtOAc-hexanes), $R f$ :

0.59 (UV, CAM). 


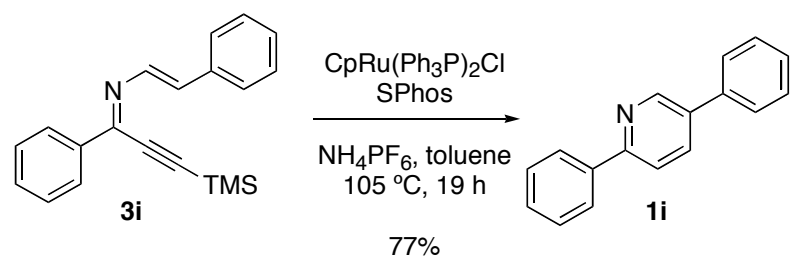

\section{2,5-Diphenyl-pyridine (1i, Table 1, entry 9): $:^{15}$}

An oven dried pressure vessel containing a magnetic stir bar was charged with ammonium hexafluorophosphate (40 mg, $0.25 \mathrm{mmol}, 1.0$ equiv), $\mathrm{CpRu}\left(\mathrm{PPh}_{3}\right)_{2} \mathrm{Cl}$ (18 mg, $0.025 \mathrm{mmol}, 0.10$ equiv) and SPhos (10 mg, $0.025 \mathrm{mmol}, 0.10$ equiv) under a nitrogen atmosphere in a glove-box and the flask sealed and brought out of the glove-box. Imine $3 \mathbf{i}(75 \mathrm{mg}, 0.25 \mathrm{mmol}, 1$ equiv) and toluene (1.3 $\mathrm{mL})$ were subsequently added via syringe. The flask was flushed with argon, sealed, stirred, and placed in an oil bath at $105^{\circ} \mathrm{C}$. After $19 \mathrm{~h}$, the reaction vessel was allowed to cool to ambient temperature and the mixture was transferred to a recovery flask with a $10-\mathrm{mL}$ portion of dichloromethane. This solution was concentrated under reduced pressure and the residue was purified by flash column chromatography on neutralized silica gel (20\% EtoAc/Hexanes) to afford the pyridine $\mathbf{1 i}$ as a pale yellow solid (44 mg, 77\%).

${ }^{1} \mathrm{H}$ NMR $\left(500 \mathrm{MHz}, \mathrm{CDCl}_{3}, 20^{\circ} \mathrm{C}\right): \quad 8.96(\mathrm{~d}, 1 \mathrm{H}, J=2.5 \mathrm{~Hz}, \operatorname{ArH}), 8.08-8.05(\mathrm{~m}, 2 \mathrm{H}, \operatorname{ArH})$, $7.98(\mathrm{dd}, 1 \mathrm{H}, J=8.2,2.5 \mathrm{~Hz}, \operatorname{ArH}), 7.83(\mathrm{~d}, 1 \mathrm{H}, J=8.2$ $\mathrm{Hz}, \mathrm{ArH}), 7.68-7.64$ (m, 2H, ArH), 7.54-7.50 (m, 4H, ArH), 7.47-7.42 (m, 2H, ArH).

${ }^{13} \mathrm{C} \mathrm{NMR}\left(125 \mathrm{MHz}, \mathrm{CDCl}_{3}, 20{ }^{\circ} \mathrm{C}\right) \delta: \quad 156.4,148.3,139.2,137.9,135.3,135.1,129.3,129.2$, $129.0,128.3,127.2,127.0,120.6$.

$\operatorname{FTIR}\left(\mathrm{CH}_{2} \mathrm{Cl}_{2}\right) \mathrm{cm}^{-1}$ : $2926(\mathrm{w}), 1735$ (s), $1654(\mathrm{~s}), 1594(\mathrm{w}), 1472(\mathrm{~s}), 1371$ (s).

HRMS (ESI):

calcd for $\mathrm{C}_{17} \mathrm{H}_{14} \mathrm{~N}[\mathrm{M}+\mathrm{H}]^{+}: 232.1121$, found: 232.1126 .

TLC (20\% EtOAc-hexanes), $R f: \quad 0.43$ (UV, CAM).

\footnotetext{
${ }^{15}$ Berthiol, F.; Kondolff, I.; Doucet, H.; Santelli, M. J. Organomet. Chem. 2004, 689, 2786-2798.
} 
<smiles>O=C(/N=C/c1cccc2ccccc12)c1ccccc1</smiles>

2j

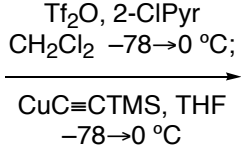

$87 \%$

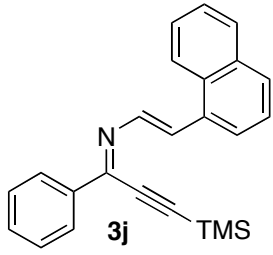

$$
\text { s }
$$

(2-Naphthalen-1-yl-vinyl)-[1-phenyl-3-(trimethyl-silanyl)-prop-2-ynylidene]-amine (3j, Table 1, entry 10):

Trifluoromethanesulfonic anhydride $(127 \mu \mathrm{L}, 0.77 \mathrm{mmol}, 1.20$ equiv) was added via syringe over $1 \mathrm{~min}$ to a stirred mixture of amide $2 \mathbf{j}$ ( $175 \mathrm{mg}, 0.64 \mathrm{mmol}, 1$ equiv) and 2-chloropyridine (242 $\mu \mathrm{L}, 2.56 \mathrm{mmol}, 4.00$ equiv) in $\mathrm{CH}_{2} \mathrm{Cl}_{2}(1.3 \mathrm{~mL})$ at $-78^{\circ} \mathrm{C}$. After $5 \mathrm{~min}$, the reaction mixture was warmed to $0{ }^{\circ} \mathrm{C}$. After $20 \mathrm{~min}$, the solution was cooled to $-78^{\circ} \mathrm{C}$ and a freshly prepared solution of copper (I) (trimethylysilyl)-acetylide $\left(278 \mathrm{mg}, 1.73 \mathrm{mmol}, 2.70\right.$ equiv) in THF $(4.0 \mathrm{~mL})$ at $0^{\circ} \mathrm{C}$ was added via cannula. The reaction mixture was kept at $-78^{\circ} \mathrm{C}$ for $5 \mathrm{~min}$ and then warmed to $0{ }^{\circ} \mathrm{C}$. After $10 \mathrm{~min}$, the crude reaction mixture was filtered through celite $(2 \mathrm{~cm} \mathrm{diam.} \times 3 \mathrm{~cm} \mathrm{ht}$. $)$ and the filtrate was concentrated under reduced pressure. The residue was purified by flash column chromatography on silica gel $(100 \%$ hexanes $\rightarrow 7.5 \%$ EtOAc/hexanes) to afford the alkynyl imine $\mathbf{3 j}$ as a pale yellow oil (197 $\mathrm{mg}, 87 \%)$.

${ }^{1} \mathrm{H} \mathrm{NMR}\left(500 \mathrm{MHz}, \mathrm{CDCl}_{3}, 20^{\circ} \mathrm{C}\right)$ :

$8.39(\mathrm{dd}, 1 \mathrm{H}, J=12.8,0.6 \mathrm{~Hz}, \mathrm{NCHCHC}), 8.31(\mathrm{~d}, 1 \mathrm{H}$, $J=8.2 \mathrm{~Hz}, 8.22-8.18(\mathrm{~m}, 2 \mathrm{H}, \mathrm{ArH}), 7.94(\mathrm{~d}, 1 \mathrm{H}, J=$ $13.1 \mathrm{~Hz}, \mathrm{NCHCHC}), 7.89$ (d, 1H, $J=7.9 \mathrm{~Hz}, \mathrm{ArH})$, 7.85 (d, 2H, $J=7.6 \mathrm{~Hz}, \operatorname{ArH}), 7.60-7.47$ (m, 6H, ArH), $0.38\left(\mathrm{~s}, 9 \mathrm{H}, \mathrm{Si}\left(\mathrm{CH}_{3}\right)_{3}\right)$.

${ }^{13} \mathrm{C} \mathrm{NMR}\left(125 \mathrm{MHz}, \mathrm{CDCl}_{3}, 20{ }^{\circ} \mathrm{C}\right) \delta: \quad 148.8,142.4,137.7,134.6,134.3,132.3,131.6,131.2$, $129.5,129.4,129.2,128.7,127.1,126.8,126.4,124.8$, $124.6,109.3,97.5,0.6$.

FTIR (neat) $\mathrm{cm}^{-1}$ : $3059(\mathrm{~m}), 2959(\mathrm{~m}), 2141(\mathrm{w}), 1810(\mathrm{w}), 1692(\mathrm{~m})$, 1644 (w), 1590 (w), 1251 (s).

HRMS (ESI): calcd for $\mathrm{C}_{24} \mathrm{H}_{24} \mathrm{NSi}[\mathrm{M}+\mathrm{H}]^{+}:$354.1673, found: 354.1675 .

TLC (20\% EtOAc-hexanes), Rf: 0.77 (UV, CAM). 


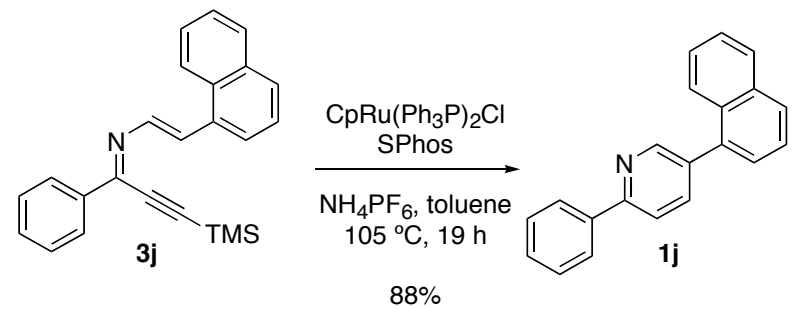

\section{5-Naphthalen-1-yl-2-phenyl-pyridine (1j, Table 1, entry 10):}

An oven dried pressure vessel containing a magnetic stir bar was charged with ammonium hexafluorophosphate (28 mg, $0.17 \mathrm{mmol}, 1.0$ equiv), $\mathrm{CpRu}\left(\mathrm{PPh}_{3}\right)_{2} \mathrm{Cl}$ (12 mg, $0.017 \mathrm{mmol}, 0.10$ equiv) and SPhos (7 mg, $0.017 \mathrm{mmol}, 0.10$ equiv) under a nitrogen atmosphere in a glove-box and the flask sealed and brought out of the glove-box. Imine $3 \mathbf{j}(60 \mathrm{mg}, 0.17 \mathrm{mmol}, 1$ equiv) and toluene $(0.9 \mathrm{~mL})$ were subsequently added via syringe. The flask was flushed with argon, sealed, stirred, and placed in an oil bath at $105^{\circ} \mathrm{C}$. After $19 \mathrm{~h}$, the reaction vessel was allowed to cool to ambient temperature and the mixture was transferred to a recovery flask with a $10-\mathrm{mL}$ portion of dichloromethane. This solution was concentrated under reduced pressure and the residue was purified by flash column chromatography on neutralized silica gel (7.5\% EtoAc/Hexanes) to afford the pyridine $\mathbf{1} \mathbf{j}$ as a pale brown solid (44 mg, 88\%).

${ }^{1} \mathrm{H} \mathrm{NMR}\left(500 \mathrm{MHz}, \mathrm{CDCl}_{3}, 20^{\circ} \mathrm{C}\right)$ :

${ }^{13} \mathrm{C}$ NMR $\left(125 \mathrm{MHz}, \mathrm{CDCl}_{3}, 20^{\circ} \mathrm{C}\right)$ :

FTIR (neat):

HRMS (ESI):

TLC (15\% EtOAc-hexanes), Rf:
8.87-8.84 (m, 1H, ArH), 8.11 (d, 2H, $J=7.02 \mathrm{~Hz}, \operatorname{ArH})$, 7.98-7.87 (m, 5H, ArH), 7.61-7.46 (m, 7H, ArH).

$156.4,150.6,139.3,138.4,136.4,134.9,134.0,131.7$, $129.3,129.0,128.7,128.7,127.6,127.1,126.7,126.3$, $125.6,125.6,120.1$.

3058 (m), 2930 (w), 1950 (w), 1595 (m), 1548 (m), $1476(\mathrm{~s}), 1396(\mathrm{~m})$.

calcd for $\mathrm{C}_{21} \mathrm{H}_{16} \mathrm{~N}[\mathrm{M}+\mathrm{H}]^{+}$: 282.1277, found: 282.1289 .

$0.46\left(\mathrm{UV}, \mathrm{KMnO}_{4}\right)$. 


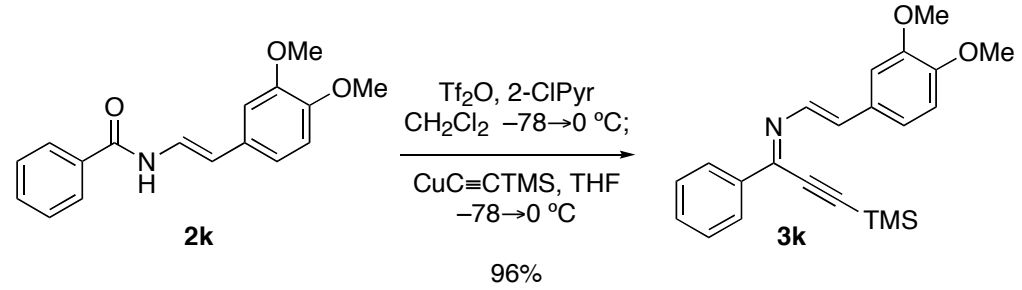

[2-(3,4-Dimethoxy-phenyl)-vinyl]-[1-phenyl-3-(trimethyl-silanyl)-prop-2-ynylidene]-amine (3k, Table 1, entry 11):

Trifluoromethanesulfonic anhydride $(122 \mu \mathrm{L}, 0.74 \mathrm{mmol}, 1.20$ equiv) was added via syringe over $1 \mathrm{~min}$ to a stirred mixture of amide $2 \mathbf{k}$ (175 $\mathrm{mg}, 0.62 \mathrm{mmol}, 1$ equiv) and 2-chloropyridine (234 $\mu \mathrm{L}, 2.47 \mathrm{mmol}, 4.00$ equiv) in $\mathrm{CH}_{2} \mathrm{Cl}_{2}(1.2 \mathrm{~mL})$ at $-78^{\circ} \mathrm{C}$. After $5 \mathrm{~min}$, the reaction mixture was warmed to $0{ }^{\circ} \mathrm{C}$. After $20 \mathrm{~min}$, the solution was cooled to $-78^{\circ} \mathrm{C}$ and a freshly prepared solution of copper (I) (trimethylysilyl)-acetylide $\left(268 \mathrm{mg}, 1.67 \mathrm{mmol}, 2.70\right.$ equiv) in THF $(4.0 \mathrm{~mL})$ at $0{ }^{\circ} \mathrm{C}$ was added via cannula. The reaction mixture was kept at $-78{ }^{\circ} \mathrm{C}$ for $5 \mathrm{~min}$ and then warmed to $0{ }^{\circ} \mathrm{C}$. After $10 \mathrm{~min}$, the crude reaction mixture was filtered through celite $(2 \mathrm{~cm} \mathrm{diam} . \times 3 \mathrm{~cm} \mathrm{ht}$. $)$ and the filtrate was concentrated under reduced pressure. The residue was purified by flash column chromatography on silica gel (15\% EtOAc/hexanes) to afford the alkynyl imine 3k as a yellow oil (216 mg, 96\%).

${ }^{1} \mathrm{H}$ NMR $\left(500 \mathrm{MHz}, \mathrm{CDCl}_{3}, 20^{\circ} \mathrm{C}\right)$ :

$8.26(\mathrm{~d}, 1 \mathrm{H}, J=13.1 \mathrm{~Hz}, \mathrm{NCHCHC}), 8.16-8.12(\mathrm{~m}, 2 \mathrm{H}$, NCHCHC, ArH), 7.46-7.42 (m, 3H, ArH), 7.14-7.08 $(\mathrm{m}, 3 \mathrm{H}, \operatorname{ArH}), 6.86(\mathrm{~d}, 1 \mathrm{H}, J=8.2 \mathrm{~Hz}, \operatorname{ArH}), 3.96(\mathrm{~s}$, $\left.3 \mathrm{H}, \mathrm{OCH}_{3}\right), 3.94\left(\mathrm{~s}, 3 \mathrm{H}, \mathrm{OCH}_{3}\right), 0.38\left(\mathrm{~s}, 9 \mathrm{H}, \mathrm{Si}\left(\mathrm{CH}_{3}\right)_{3}\right)$.

${ }^{13} \mathrm{C}$ NMR $\left(125 \mathrm{MHz}, \mathrm{CDCl}_{3}, 20^{\circ} \mathrm{C}\right) \delta:$

FTIR (neat) $\mathrm{cm}^{-1}$ :

HRMS (ESI):

TLC (20\% EtOAc-hexanes), Rf:
$149.6,149.2,146.6,138.2,137.1,133.8,130.7,129.6$, $128.5,127.8,121.4,111.3,108.8,108.1,96.9,56.1,55.8$, -0.0 .

3057 (w), 2958 (m), 2835 (w), 1599 (s), 1578 (m), 1512 (s), 1267 (s).

calcd for $\mathrm{C}_{22} \mathrm{H}_{26} \mathrm{NO}_{2} \mathrm{Si}[\mathrm{M}+\mathrm{H}]^{+}: 364.1727$, found: 364.1733 .

$0.52(\mathrm{UV}, \mathrm{CAM})$. 


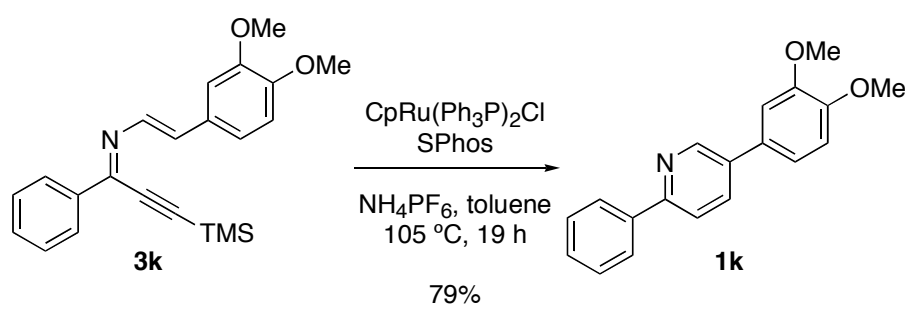

\section{5-(3,4-Dimethoxy-phenyl)-2-phenyl-pyridine (1 k, Table 1, entry 11):}

An oven dried pressure vessel containing a magnetic stir bar was charged with ammonium hexafluorophosphate (45 mg, $0.28 \mathrm{mmol}, 1.0$ equiv), $\mathrm{CpRu}\left(\mathrm{PPh}_{3}\right)_{2} \mathrm{Cl}$ (20 mg, $0.028 \mathrm{mmol}, 0.10$ equiv) and SPhos (11 mg, $0.028 \mathrm{mmol}, 0.10$ equiv) under a nitrogen atmosphere in a glove-box and the flask sealed and brought out of the glove-box. Imine 3k (100 mg, $0.28 \mathrm{mmol}, 1$ equiv) and toluene $(1.4 \mathrm{~mL})$ were subsequently added via syringe. The flask was flushed with argon, sealed, stirred, and placed in an oil bath at $105^{\circ} \mathrm{C}$. After $19 \mathrm{~h}$, the reaction vessel was allowed to cool to ambient temperature and the mixture was transferred to a recovery flask with a $10-\mathrm{mL}$ portion of dichloromethane. This solution was concentrated under reduced pressure and the residue was purified by flash column chromatography on neutralized silica gel (30\% EtoAc/Hexanes) to afford the pyridine $\mathbf{1 k}$ as a yellow solid (64 mg, 79\%).

${ }^{1} \mathrm{H}$ NMR (500 MHz, $\left.\mathrm{CDCl}_{3}, 20^{\circ} \mathrm{C}\right)$ :

${ }^{13} \mathrm{C} \mathrm{NMR}\left(125 \mathrm{MHz}, \mathrm{CDCl}_{3}, 20^{\circ} \mathrm{C}\right)$ :

FTIR (neat):

HRMS (ESI):

TLC (20\% EtOAc-hexanes), Rf: $8.92(\mathrm{~d}, 1 \mathrm{H}, J=2.1 \mathrm{~Hz}, \operatorname{ArH}), 8.07-8.03$ (m, 2H, ArH), $7.93(\mathrm{dd}, 1 \mathrm{H}, J=8.2,2.5 \mathrm{~Hz}, \operatorname{ArH}), 7.81(\mathrm{~d}, 1 \mathrm{H}, J=8.2$ $\mathrm{Hz}, \operatorname{ArH}), 7.53-7.49$ (m, 2H, ArH), 7.44 (tt, 1H, $J=7.3$, $1.1 \mathrm{~Hz}, \operatorname{ArH}), 7.22(\mathrm{dd}, 1 \mathrm{H}, J=8.2,2.1 \mathrm{~Hz}, \operatorname{ArH}), 7.15$ $(\mathrm{d}, 1 \mathrm{H}, J=1.8 \mathrm{~Hz}, \operatorname{ArH}), 7.02(\mathrm{~d}, 1 \mathrm{H}, J=8.2 \mathrm{~Hz}, \operatorname{ArH})$, $3.99\left(\mathrm{~s}, 3 \mathrm{H}, \mathrm{OCH}_{3}\right), 3.96\left(\mathrm{~s}, 3 \mathrm{H}, \mathrm{OCB}_{3}\right)$.

$155.7,149.5,149.3,147.9,139.1,134.8,134.8,130.5$, 129.0, 128.9, 126.8, 120.3, 119.5, 111.8, 110.1, 56.1, 56.1 .

$3055(\mathrm{w}), 3005(\mathrm{w}), 2966(\mathrm{~m}), 2837(\mathrm{~m}), 1602$ (s), 1590 (s), 1522 (s), 1150 (s), 1022 (s).

calcd for $\mathrm{C}_{19} \mathrm{H}_{18} \mathrm{NO}_{2}[\mathrm{M}+\mathrm{H}]^{+}$: 292.1332, found: 292.1337 .

$0.19\left(\mathrm{UV}, \mathrm{KMnO}_{4}\right)$. 


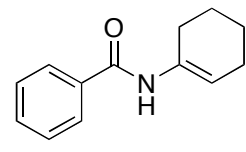

2l

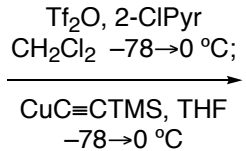

$75 \%$

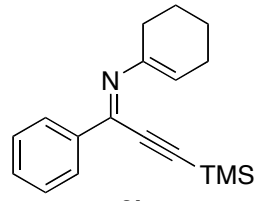

31

\section{$N$-(Cyclohexen-1-yl)-2-phenyl-4-trimethylsilyl-1-azabut-1-en-3-yne (3l, Table 1, entry 12):}

Trifluoromethanesulfonic anhydride $(148 \mu \mathrm{L}, 0.89 \mathrm{mmol}, 1.20$ equiv) was added via syringe over $1 \mathrm{~min}$ to a stirred mixture of amide 21 (150 $\mathrm{mg}, 0.75 \mathrm{mmol}, 1$ equiv) and 2-chloropyridine (282 $\mu \mathrm{L}, 2.98 \mathrm{mmol}, 4.00$ equiv) in $\mathrm{CH}_{2} \mathrm{Cl}_{2}(1.5 \mathrm{~mL})$ at $-78{ }^{\circ} \mathrm{C}$. After $5 \mathrm{~min}$, the reaction mixture was warmed to $0{ }^{\circ} \mathrm{C}$. After $20 \mathrm{~min}$, the solution was cooled to $-78^{\circ} \mathrm{C}$ and a freshly prepared solution of copper (I) (trimethylysilyl)-acetylide (323 mg, $2.01 \mathrm{mmol}, 2.70$ equiv) in THF $(3.0 \mathrm{~mL})$ at $0{ }^{\circ} \mathrm{C}$ was added via cannula. The reaction mixture was kept at $-78{ }^{\circ} \mathrm{C}$ for $5 \mathrm{~min}$ and then warmed to $0{ }^{\circ} \mathrm{C}$. After $10 \mathrm{~min}$, the crude reaction mixture was filtered through celite $(2 \mathrm{~cm}$ diam. $\times 3 \mathrm{~cm} \mathrm{ht}$.) and the filtrate was concentrated under reduced pressure. The residue was purified by flash column chromatography on silica gel $(100 \%$ hexanes $\rightarrow 7.5 \%$ EtOAc/hexanes) to afford the alkynyl imine 31 as a yellow oil (157 mg, 75\%).

${ }^{1} \mathrm{H}$ NMR $\left(500 \mathrm{MHz}, \mathrm{CDCl}_{3}, 20{ }^{\circ} \mathrm{C}\right) \delta:$

${ }^{13} \mathrm{C}$ NMR $\left(125 \mathrm{MHz}, \mathrm{CDCl}_{3}, 20^{\circ} \mathrm{C}\right) \delta:$

FTIR (neat) $\mathrm{cm}^{-1}$ :

HRMS (ESI):

TLC (20\% EtOAc/hexanes), Rf:
8.09-8.05 (m, 2H, ArH), 7.48-7.39 (m, 3H, ArH), 5.21 (t, $1 \mathrm{H}, J=4.0 \mathrm{~Hz}, \mathrm{NC}=\mathrm{CH}), 2.29-2.23\left(\mathrm{~m}, 2 \mathrm{H}, \mathrm{CH}_{2}\right)$, 2.22-2.16 (m, 2H, CH $), 1.82-1.75\left(\mathrm{~m}, 2 \mathrm{H}, \mathrm{CH}_{2}\right), 1.70$ $1.64\left(\mathrm{~m}, 2 \mathrm{H}, \mathrm{CH}_{2}\right), 0.28\left(\mathrm{~s}, 9 \mathrm{H}, \mathrm{Si}\left(\mathrm{CH}_{3}\right)_{3}\right)$.

$148.7,147.5,137.2,130.8,128.4,128.0,110.3,103.7$, $97.6,27.9,24.7,23.1,22.5,-0.1$.

3063 (w), 2927 (s), 2857 (m), 1663 (m), 1562 (m), 1448 (m), 1273 (s), 1251 (s).

calcd for $\mathrm{C}_{18} \mathrm{H}_{24} \mathrm{NSi}[\mathrm{M}+\mathrm{H}]^{+}: 282.1673$, found: 282.1685 .

$0.61\left(\mathrm{UV}, \mathrm{KMnO}_{4}\right)$. 


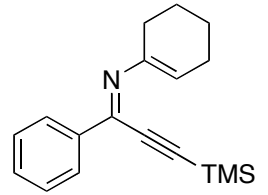

3)

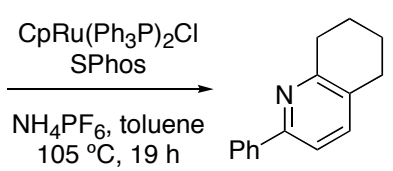

11

\section{2-Phenyl-5,6,7,8-tetrahydro-quinoline (11, Table 1, entry 12):}

An oven dried pressure vessel containing a magnetic stir bar was charged with ammonium hexafluorophosphate (43 mg, $0.27 \mathrm{mmol}, 1.0$ equiv), $\mathrm{CpRu}\left(\mathrm{PPh}_{3}\right)_{2} \mathrm{Cl}$ (19 mg, $0.027 \mathrm{mmol}, 0.10$ equiv) and SPhos (11 mg, $0.027 \mathrm{mmol}, 0.10$ equiv) under a nitrogen atmosphere in a glove-box and the flask sealed and brought out of the glove-box. Imine 31 ( $75 \mathrm{mg}, 0.27 \mathrm{mmol}, 1$ equiv) and toluene $(1.3 \mathrm{~mL})$ were subsequently added via syringe. The flask was flushed with argon, sealed, stirred, and placed in an oil bath at $105^{\circ} \mathrm{C}$. After $19 \mathrm{~h}$, the reaction vessel was allowed to cool to ambient temperature and the mixture was transferred to a recovery flask with a $10-\mathrm{mL}$ portion of dichloromethane. This solution was concentrated under reduced pressure and the residue was purified by flash column chromatography on silica gel (20\% EtoAc/Hexanes) to afford the pyridine $\mathbf{1 1}$ as a pale yellow solid (41 mg, 73\%).

${ }^{1} \mathrm{H}$ NMR $\left(500 \mathrm{MHz}, \mathrm{CDCl}_{3}, 20^{\circ} \mathrm{C}\right)$ :

${ }^{13} \mathrm{C} \mathrm{NMR}\left(125 \mathrm{MHz}, \mathrm{CDCl}_{3}, 20^{\circ} \mathrm{C}\right)$ :

FTIR (neat):

HRMS (ESI):

TLC (20\% EtOAc-hexanes), Rf:
7.97-7.94 (m, 2H, ArH), 7.48-7.36 (m, 5H, ArH), 3.00 $\left(\mathrm{t}, 2 \mathrm{H}, J=6.4 \mathrm{~Hz}, \mathrm{CH}_{2}\right), 2.82\left(\mathrm{t}, 2 \mathrm{H}, J=6.4 \mathrm{~Hz}, \mathrm{CH}_{2}\right)$, 1.98-1.92 (m, 2H, CH$), 1.89-1.83\left(\mathrm{~m}, 2 \mathrm{H}, \mathrm{CH}_{2}\right)$.

$157.4,154.9,140.1,137.6,130.9,128.8,128.5,127.0$, $118.1,33.1,28.8,23.4,23.0$.

$3061(\mathrm{w}), 3032(\mathrm{w}), 2935(\mathrm{~s}), 2860(\mathrm{~m}), 1590(\mathrm{~m}), 1566$ (m), 1460 (s), 1434 (m), 1253 (m).

calcd for $\mathrm{C}_{15} \mathrm{H}_{16} \mathrm{~N}[\mathrm{M}+\mathrm{H}]^{+}: 210.1277$, found: 210.1279 .

$0.48\left(\mathrm{UV}, \mathrm{KMnO}_{4}\right)$. 


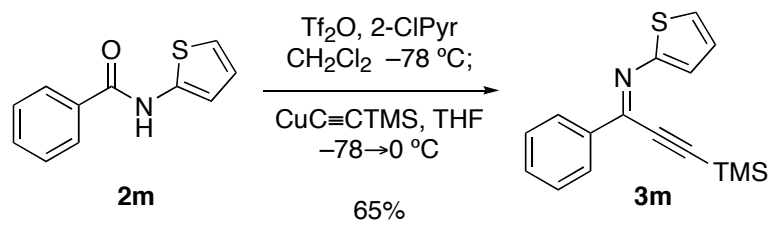

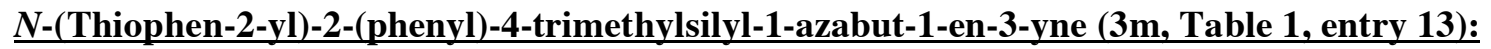

Trifluoromethanesulfonic anhydride $(195 \mu \mathrm{L}, 1.18 \mathrm{mmol}, 1.20$ equiv) was added via syringe over $1 \mathrm{~min}$ to a stirred mixture of amide $\mathbf{2 m}$ (200 $\mathrm{mg}, 0.98 \mathrm{mmol}, 1$ equiv) and 2-chloropyridine (372 $\mu \mathrm{L}, 3.94 \mathrm{mmol}, 4.00$ equiv) in $\mathrm{CH}_{2} \mathrm{Cl}_{2}(2.0 \mathrm{~mL})$ at $-78^{\circ} \mathrm{C}$. After $10 \mathrm{~min}$, a freshly prepared solution of copper (I) (trimethylysilyl)-acetylide (428 mg, $2.66 \mathrm{mmol}, 2.70$ equiv) in THF $(5.0 \mathrm{~mL})$ at $0{ }^{\circ} \mathrm{C}$ was added via cannula. The reaction mixture was kept at $-78^{\circ} \mathrm{C}$ for $10 \mathrm{~min}$ and then warmed to $0{ }^{\circ} \mathrm{C}$. After $10 \mathrm{~min}$, the crude reaction mixture was concentrated under reduced pressure. The residue was purified by flash column chromatography on silica gel $(100 \%$ hexanes $\rightarrow 7.5 \%$ EtOAc/hexanes $)$ to afford the alkynyl imine $\mathbf{3 m}$ as a yellow oil (181 $\mathrm{mg}, 65 \%)$.

${ }^{1} \mathrm{H}$ NMR $\left(500 \mathrm{MHz}, \mathrm{CDCl}_{3}, 20^{\circ} \mathrm{C}\right) \delta: \quad 8.19-8.13(\mathrm{~m}, 2 \mathrm{H}, \operatorname{ArH}), 7.48-7.43(\mathrm{~m}, 3 \mathrm{H}, \operatorname{ArH}), 7.42$ (dd, $1 \mathrm{H}, J=3.8,1.4 \mathrm{~Hz}, \mathrm{CHS}), 7.32(\mathrm{dd}, 1 \mathrm{H}, J=5.5$, $1.4 \mathrm{~Hz}, \mathrm{CHCHCHS}), 7.07$ (dd, $1 \mathrm{H}, J=5.5,3.8 \mathrm{~Hz}$, CHCHS), 0.40 (s, 9H, $\left.\mathrm{Si}\left(\mathrm{CH}_{3}\right)_{3}\right)$.

${ }^{13} \mathrm{C} \mathrm{NMR}\left(125 \mathrm{MHz}, \mathrm{CDCl}_{3}, 20{ }^{\circ} \mathrm{C}\right) \delta: \quad 152.2,140.9,137.7,130.6,129.2,128.5,127.9,125.4$, $125.3,112.0,99.2,-0.6$.

FTIR (neat) $\mathrm{cm}^{-1}$ :

HRMS (ESI):

TLC (20\% EtOAc-hexanes), Rf: $3064(\mathrm{~m}), 2960(\mathrm{~s}), 2144(\mathrm{~m}), 2067$ (m), 1537 (m), 1412 (s), $1272(\mathrm{~s}), 1253(\mathrm{~s})$.

calcd for $\mathrm{C}_{16} \mathrm{H}_{18} \mathrm{NSSi}[\mathrm{M}+\mathrm{H}]^{+}$: 284.0924, found: 284.0934 .

0.63 (UV, CAM). 

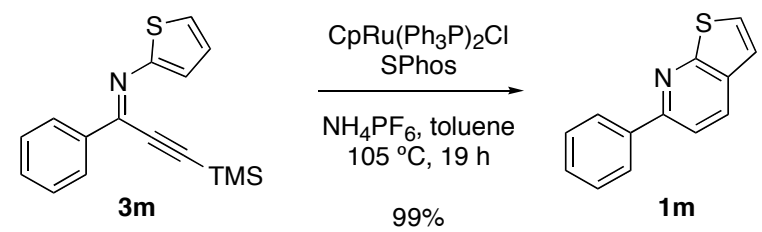

\section{6-Phenyl-thieno[2,3-b]pyridine (1 $\mathrm{m}$, Table 1, entry 13):}

An oven dried pressure vessel containing a magnetic stir bar was charged with ammonium hexafluorophosphate (35 mg, $0.21 \mathrm{mmol}, 1.0$ equiv), $\mathrm{CpRu}\left(\mathrm{PPh}_{3}\right)_{2} \mathrm{Cl}$ (15 mg, $0.021 \mathrm{mmol}, 0.10$ equiv) and SPhos ( $9 \mathrm{mg}, 0.021 \mathrm{mmol}, 0.10$ equiv) under a nitrogen atmosphere in a glove-box and the flask sealed and brought out of the glove-box. Imine $1 \mathrm{~m}(60 \mathrm{mg}, 0.21 \mathrm{mmol}, 1$ equiv) and toluene $(1.1 \mathrm{~mL})$ were subsequently added via syringe. The flask was flushed with argon, sealed, stirred, and placed in an oil bath at $105^{\circ} \mathrm{C}$. After $19 \mathrm{~h}$, the reaction vessel was allowed to cool to ambient temperature and the mixture was transferred to a recovery flask with a $10-\mathrm{mL}$ portion of dichloromethane. This solution was concentrated under reduced pressure and the residue was purified by flash column chromatography on silica gel (20\% EtoAc/Hexanes) to afford the thienopyridine $\mathbf{1} \mathbf{m}^{16}$ as a pale yellow solid (44 mg, 99\%).

${ }^{1} \mathrm{H}$ NMR $\left(500 \mathrm{MHz}, \mathrm{CDCl}_{3}, 20^{\circ} \mathrm{C}\right)$ :

${ }^{13} \mathrm{C}$ NMR $\left(125 \mathrm{MHz}, \mathrm{CDCl}_{3}, 20^{\circ} \mathrm{C}\right)$ :

$\operatorname{FTIR}\left(\mathrm{CH}_{2} \mathrm{Cl}_{2}\right)$ :

HRMS (ESI):

TLC (20\% EtOAc-hexanes), Rf: $8.15(\mathrm{~d}, 1 \mathrm{H}, J=8.2 \mathrm{~Hz}, \operatorname{ArH}), 8.11-8.09(\mathrm{~m}, 2 \mathrm{H}, \operatorname{ArH})$, 7.78 (d, 1H, $J=8.6 \mathrm{~Hz}, \operatorname{ArH}), 7.54-7.49$ (m, 3H, ArH, $\mathrm{SCH}), 7.44(\mathrm{tt}, 1 \mathrm{H}, J=7.3,1.2 \mathrm{~Hz}, \operatorname{ArH}), 7.30(\mathrm{~d}, 1 \mathrm{H}, J$ $=5.8 \mathrm{~Hz}, \mathrm{SCHCH})$.

$162.4,154.7,139.4,131.7,131.3,129.1,129.0,127.4$, $127.2,121.5,117.0$.

3025 (w), 1714 (w), 1574 (s), 1558 (m), 1480 (m), 1333 (m).

calcd for $\mathrm{C}_{13} \mathrm{H}_{10} \mathrm{NS}[\mathrm{M}+\mathrm{H}]^{+}:$212.0534, found: 212.0535 .

$0.45\left(\mathrm{UV}, \mathrm{KMnO}_{4}\right)$.

\footnotetext{
${ }^{16}$ Taylor, E. C.; Macor, J. E. J. Org. Chem. 1987, 52, 4280-4287.
} 

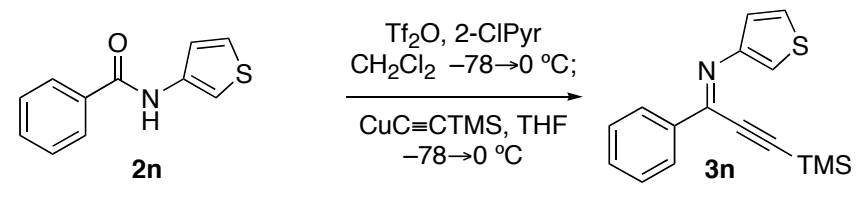

$98 \%$

\section{[1-Phenyl-3-(trimethyl-silanyl)-prop-2-ynylidene]-thiophen-3-yl-amine (3n, Table 1, entry 14):}

Trifluoromethanesulfonic anhydride $(294 \mu \mathrm{L}, 1.77 \mathrm{mmol}, 1.20$ equiv) was added via syringe over $1 \mathrm{~min}$ to a stirred mixture of amide $2 \mathrm{n}$ (300 $\mathrm{mg}, 1.48 \mathrm{mmol}, 1$ equiv) and 2-chloropyridine (559 $\mu \mathrm{L}, 5.90 \mathrm{mmol}, 4.00$ equiv) in $\mathrm{CH}_{2} \mathrm{Cl}_{2}(3.0 \mathrm{~mL})$ at $-78{ }^{\circ} \mathrm{C}$. After $5 \mathrm{~min}$, the reaction mixture was warmed to $0{ }^{\circ} \mathrm{C}$. After $20 \mathrm{~min}$, the solution was cooled to $-78^{\circ} \mathrm{C}$ and a freshly prepared solution of copper (I) (trimethylysilyl)-acetylide $\left(641 \mathrm{mg}, 3.99 \mathrm{mmol}, 2.70\right.$ equiv) in THF $(5.0 \mathrm{~mL})$ at $0{ }^{\circ} \mathrm{C}$ was added via cannula. The reaction mixture was kept at $-78{ }^{\circ} \mathrm{C}$ for $5 \mathrm{~min}$ and then warmed to $0{ }^{\circ} \mathrm{C}$. After $10 \mathrm{~min}$, the crude reaction mixture was filtered through celite $(2 \mathrm{~cm} \mathrm{diam} . \times 3 \mathrm{~cm} \mathrm{ht}$. $)$ and the filtrate was concentrated under reduced pressure. The residue was purified by flash column chromatography on silica gel $(100 \%$ hexanes $\rightarrow 10 \%$ EtOAc/hexanes) to afford the alkynyl imine $3 \mathrm{n}$ as a yellow oil (411 mg, 98\%).

${ }^{1} \mathrm{H}$ NMR $\left(500 \mathrm{MHz}, \mathrm{CDCl}_{3}, 20{ }^{\circ} \mathrm{C}\right) \delta:$

${ }^{13} \mathrm{C}$ NMR $\left(125 \mathrm{MHz}, \mathrm{CDCl}_{3}, 20^{\circ} \mathrm{C}\right) \delta$ :

FTIR (neat) $\mathrm{cm}^{-1}$ :

HRMS (ESI):

TLC (20\% EtOAc/hexanes), Rf:
8.17 (dd, 2H, $J=7.6,1.8 \mathrm{~Hz}, \operatorname{ArH}), 7.50-7.43$ (m, 4H, ArH, CHS)), 7.38 (d, 1H, J=5.2 Hz, CHS), 7.31 (dd, $1 \mathrm{H}, J=5.2,3.4 \mathrm{~Hz}, \mathrm{CHCHS}), 0.29$ (s, 9H, $\left.\mathrm{Si}\left(\mathrm{CH}_{3}\right)_{3}\right)$.

$149.9,147.4,137.6,131.0,128.5,128.1,124.9,124.2$, $115.6,106.1,98.8,-0.4$.

3740 (w), 3108 (m), 3063 (m), 2960 (s), 2899 (m), 2147

(m), 1645 (w), 1586 (m), 1556 (s), 1271 (s).

calcd for $\mathrm{C}_{16} \mathrm{H}_{18} \mathrm{NSSi}[\mathrm{M}+\mathrm{H}]^{+}$: 284.0929, found: 284.0933 .

$0.74\left(\mathrm{UV}, \mathrm{KMnO}_{4}\right)$. 

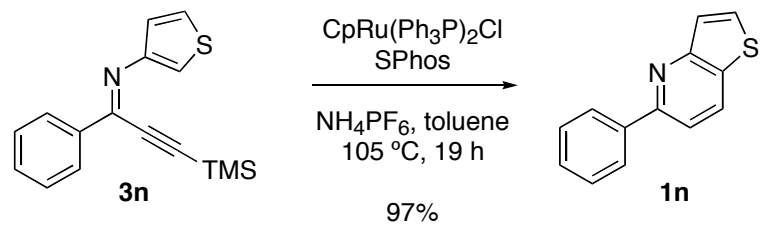

\section{5-Phenyl-thieno[3,2-b]pyridine (1n, Table 1, entry 14):}

An oven dried pressure vessel containing a magnetic stir bar was charged with ammonium hexafluorophosphate (58 mg, $0.35 \mathrm{mmol}, 1.0$ equiv), $\mathrm{CpRu}\left(\mathrm{PPh}_{3}\right)_{2} \mathrm{Cl}$ (26 mg, $0.035 \mathrm{mmol}, 0.10$ equiv) and SPhos (15 mg, $0.035 \mathrm{mmol}, 0.10$ equiv) under a nitrogen atmosphere in a glove-box and the flask sealed and brought out of the glove-box. Imine 3n (100 mg, $0.35 \mathrm{mmol}, 1$ equiv) and toluene $(1.8 \mathrm{~mL})$ were subsequently added via syringe. The flask was flushed with argon, sealed, stirred, and placed in an oil bath at $105^{\circ} \mathrm{C}$. After $19 \mathrm{~h}$, the reaction vessel was allowed to cool to ambient temperature and the mixture was transferred to a recovery flask with a $10-\mathrm{mL}$ portion of dichloromethane. This solution was concentrated under reduced pressure and the residue was purified by flash column chromatography on silica gel (15\% EtoAc/Hexanes) to afford the thienopyridine 1n as a pale yellow solid (73 mg, 97\%).

${ }^{1} \mathrm{H} \mathrm{NMR}\left(500 \mathrm{MHz}, \mathrm{CDCl}_{3}, 20^{\circ} \mathrm{C}\right)$ :

${ }^{13} \mathrm{C} \mathrm{NMR}\left(125 \mathrm{MHz}, \mathrm{CDCl}_{3}, 20^{\circ} \mathrm{C}\right)$ :

FTIR (neat):

HRMS (ESI):

TLC (20\% EtOAc-hexanes), Rf: $8.26(\mathrm{~d}, 1 \mathrm{H}, J=8.6 \mathrm{~Hz}, \operatorname{ArH}), 8.08(\mathrm{~d}, 1 \mathrm{H}, J=7.3 \mathrm{~Hz}$, ArH), 7.78 (d, 1H, $J=5.5 \mathrm{~Hz}$, CHS), 7.73 (d, 1H, $J=$ $8.5 \mathrm{~Hz}, \mathrm{ArH}), 7.65$ (d, 1H, $J=5.5 \mathrm{~Hz}, \mathrm{CHCHS})), 7.52(\mathrm{t}$, $2 \mathrm{H}, J=7.0 \mathrm{~Hz}, \operatorname{ArH}), 7.45$ (t, 1H, $J=7.3 \mathrm{~Hz}, \operatorname{ArH})$.

$156.4,155.5,139.8,131.7,131.0,131.0,129.0,128.9$, $127.4,125.5,116.5$.

3071 (s), 1906 (w), 1564 (s), 1544 (s), 1397 (s), 1280 (s), 1158 (s).

calcd for $\mathrm{C}_{13} \mathrm{H}_{10} \mathrm{NS}[\mathrm{M}+\mathrm{H}]^{+}:$212.0534, found: 212.0534 .

$0.53\left(\mathrm{UV}, \mathrm{KMnO}_{4}\right)$. 

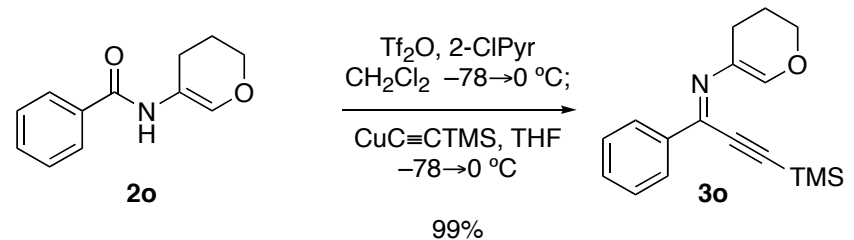

(5,6-Dihydro-4H-pyran-3-yl)-[1-phenyl-3-(trimethyl-silanyl)-prop-2-ynylidene]-amine $\quad$ (3o, Table 1 , entry 15):

Trifluoromethanesulfonic anhydride $(292 \mu \mathrm{L}, 1.77 \mathrm{mmol}, 1.20$ equiv) was added via syringe over $1 \mathrm{~min}$ to a stirred mixture of amide 20 (300 mg, $1.48 \mathrm{mmol}, 1$ equiv) and 2-chloropyridine (559 $\mu \mathrm{L}, 5.90 \mathrm{mmol}, 4.00$ equiv) in $\mathrm{CH}_{2} \mathrm{Cl}_{2}(3.0 \mathrm{~mL})$ at $-78{ }^{\circ} \mathrm{C}$. After $5 \mathrm{~min}$, the reaction mixture was warmed to $0{ }^{\circ} \mathrm{C}$. After $20 \mathrm{~min}$, the solution was cooled to $-78{ }^{\circ} \mathrm{C}$ and a freshly prepared solution of copper (I) (trimethylysilyl)-acetylide (641 mg, $3.99 \mathrm{mmol}, 2.70$ equiv) in THF $(5.0 \mathrm{~mL})$ at $0{ }^{\circ} \mathrm{C}$ was added via cannula. The reaction mixture was kept at $-78{ }^{\circ} \mathrm{C}$ for $5 \mathrm{~min}$ and then warmed to $0{ }^{\circ} \mathrm{C}$. After $10 \mathrm{~min}$, the crude reaction mixture was filtered through celite $(2 \mathrm{~cm} \mathrm{diam.} \times 3 \mathrm{~cm} \mathrm{ht}$. $)$ and the filtrate was concentrated under reduced pressure. The residue was purified by flash column chromatography on silica gel (5\% EtOAc/hexanes) to afford the alkynyl imine $\mathbf{3 0}$ as a pale yellow oil (416 mg, 99\%).

${ }^{1} \mathrm{H}$ NMR $\left(500 \mathrm{MHz}, \mathrm{C}_{6} \mathrm{D}_{6}, 20{ }^{\circ} \mathrm{C}\right) \delta$ :

${ }^{13} \mathrm{C}$ NMR $\left(125 \mathrm{MHz}, \mathrm{C}_{6} \mathrm{D}_{6}, 20{ }^{\circ} \mathrm{C}\right) \delta$ :

FTIR (neat) $\mathrm{cm}^{-1}$ :

HRMS (ESI):

TLC (20\% EtOAc/hexanes), Rf:
8.44-8.40 (m, 2H, ArH), 7.44 (bs, 1H, NC=CHO), 7.24-7.20 (m, 2H, ArH), 7.12-7.08 (m, 1H, ArH), 3.58 $\left(\mathrm{t}, 2 \mathrm{H}, J=5.2 \mathrm{~Hz}, \mathrm{CH}_{2} \mathrm{O}\right), 2.80(\mathrm{t}, 2 \mathrm{H}, J=6.4 \mathrm{~Hz}$, $\left.\mathrm{NCCH}_{2}\right), 1.46-1.40\left(\mathrm{~m}, 2 \mathrm{H}, \mathrm{CH}_{2} \mathrm{CH}_{2} \mathrm{O}\right), 0.14(\mathrm{~s}, 9 \mathrm{H}$, $\left.\mathrm{Si}\left(\mathrm{CH}_{3}\right)_{3}\right)$.

$149.7,139.9,138.2,134.3,129.9,127.9,103.2,102.1$, $100.5,66.6,24.8,22.5,-0.2$.

$3063(\mathrm{w}), 2960(\mathrm{~m}), 2920(\mathrm{~m}), 2850(\mathrm{w}), 1783(\mathrm{w})$, 1724 (s), 1675 (w), 1251 (s), 1176 (s).

calcd for $\mathrm{C}_{17} \mathrm{H}_{22} \mathrm{NOSi}[\mathrm{M}+\mathrm{H}]^{+}:$284.1465, found: 284.1471.

$0.67\left(\mathrm{UV}, \mathrm{KMnO}_{4}\right)$. 

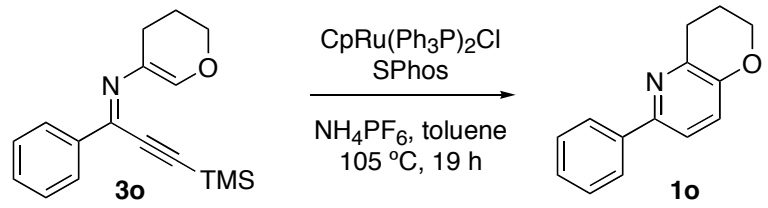

$70 \%$

\section{6-Phenyl-3,4-dihydro-2H-pyrano[3,2-b]pyridine (10, Table 1, entry 15):}

An oven dried pressure vessel containing a magnetic stir bar was charged with ammonium hexafluorophosphate (52 mg, $0.32 \mathrm{mmol}, 1.0$ equiv), $\mathrm{CpRu}\left(\mathrm{PPh}_{3}\right)_{2} \mathrm{Cl}$ (23 mg, $0.032 \mathrm{mmol}, 0.10$ equiv) and SPhos (13 mg, $0.032 \mathrm{mmol}, 0.10$ equiv) under a nitrogen atmosphere in a glove-box and the flask sealed and brought out of the glove-box. Imine 30 (90 mg, $0.32 \mathrm{mmol}, 1$ equiv) and toluene (1.6 mL) were subsequently added via syringe. The flask was flushed with argon, sealed, stirred, and placed in an oil bath at $105^{\circ} \mathrm{C}$. After $19 \mathrm{~h}$, the reaction vessel was allowed to cool to ambient temperature and the mixture was transferred to a recovery flask with a $10-\mathrm{mL}$ portion of dichloromethane. This solution was concentrated under reduced pressure and the residue was purified by flash column chromatography on silica gel $\left(1 \% \mathrm{Et}_{3} \mathrm{~N} / \mathrm{CH}_{2} \mathrm{Cl}_{2}\right)$ to afford the dihydropyranopyridine 10 as a pale yellow solid (47 mg, 70\%).

${ }^{1} \mathrm{H} \mathrm{NMR}\left(500 \mathrm{MHz}, \mathrm{CDCl}_{3}, 20^{\circ} \mathrm{C}\right)$ :

${ }^{13} \mathrm{C}$ NMR $\left(125 \mathrm{MHz}, \mathrm{C}_{6} \mathrm{D}_{6}, 20^{\circ} \mathrm{C}\right)$ :

FTIR (neat):

HRMS (EI):

TLC (20\% EtOAc-hexanes), Rf:
7.92-7.89 (m, 2H, ArH), 7.49-7.47 (m, 1H, ArH), 7.46$7.42(\mathrm{~m}, 2 \mathrm{H}, \operatorname{ArH}), 7.35(\mathrm{tt}, 1 \mathrm{H}, J=7.3,1.5 \mathrm{~Hz}, \operatorname{ArH})$, $7.16(\mathrm{~d}, 1 \mathrm{H}, J=8.6, \operatorname{ArH}), 4.24(\mathrm{t}, 2 \mathrm{H}, J=5.5 \mathrm{~Hz}$, $\left.\mathrm{CH}_{2} \mathrm{O}\right), 3.05\left(\mathrm{t}, 2 \mathrm{H}, J=6.6 \mathrm{~Hz}, \mathrm{NCCH}_{2}\right), 2.19-2.14(\mathrm{~m}$, $\left.2 \mathrm{H}, \mathrm{CH}_{2} \mathrm{CH}_{2} \mathrm{O}\right)$.

$151.5,149.6,144.0,140.3,129.2,128.5,127.1,124.6$, $119.6,66.6,29.2,22.8$.

$3061(\mathrm{w}), 3034(\mathrm{w}), 2949(\mathrm{~m}), 2874(\mathrm{~m}), 1575(\mathrm{~m})$, 1471 (s), 1458 (s), 1258 (s).

calcd for $\mathrm{C}_{14} \mathrm{H}_{13} \mathrm{NO}[\mathrm{M}]^{+}: 211.0992$, found: 211.0987.

$0.40\left(\mathrm{UV}, \mathrm{KMnO}_{4}\right)$. 


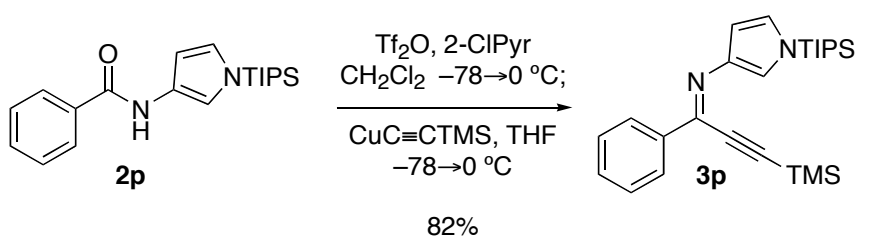

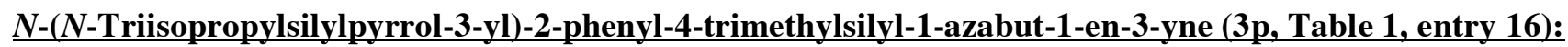

Trifluoromethanesulfonic anhydride $(175 \mu \mathrm{L}, 1.05 \mathrm{mmol}, 1.20$ equiv) was added via syringe over $1 \mathrm{~min}$ to a stirred mixture of amide $\mathbf{2 p}$ (300 $\mathrm{mg}, 0.88 \mathrm{mmol}, 1$ equiv) and 2-chloropyridine (332 $\mu \mathrm{L}, 3.50 \mathrm{mmol}, 4.00$ equiv) in $\mathrm{CH}_{2} \mathrm{Cl}_{2}(1.8 \mathrm{~mL})$ at $-78{ }^{\circ} \mathrm{C}$. After $5 \mathrm{~min}$, the reaction mixture was warmed to $0{ }^{\circ} \mathrm{C}$. After $20 \mathrm{~min}$, the solution was cooled to $-78{ }^{\circ} \mathrm{C}$ and a freshly prepared solution of copper (I) (trimethylysilyl)-acetylide $\left(380 \mathrm{mg}, 2.37 \mathrm{mmol}, 2.70\right.$ equiv) in THF $(5.0 \mathrm{~mL})$ at $0{ }^{\circ} \mathrm{C}$ was added via cannula. The reaction mixture was kept at $-78{ }^{\circ} \mathrm{C}$ for $5 \mathrm{~min}$ and then warmed to $0{ }^{\circ} \mathrm{C}$. After $10 \mathrm{~min}$, the crude reaction mixture was filtered through celite $(2 \mathrm{~cm}$ diam. $\times 3 \mathrm{~cm} \mathrm{ht}$.) and the filtrate was concentrated under reduced pressure. The residue was purified by flash column chromatography on silica gel $(100 \%$ hexanes $\rightarrow 10 \%$ EtOAc/hexanes) to afford the alkynyl imine $\mathbf{3 p}$ as a yellow oil (304 $\mathrm{mg}, 82 \%)$.

${ }^{1} \mathrm{H}$ NMR $\left(500 \mathrm{MHz}, \mathrm{C}_{6} \mathrm{D}_{6}, 20{ }^{\circ} \mathrm{C}\right) \delta: \quad 8.63-8.60(\mathrm{~m}, 2 \mathrm{H}, \mathrm{ArH}), 7.74(\mathrm{dd}, 1 \mathrm{H}, J=3.1,1.2 \mathrm{~Hz}$, CHN), $7.50(\mathrm{dd}, 1 \mathrm{H}, J=2.4,1.5 \mathrm{~Hz}, \mathrm{CHN}), 7.29-7.24$ (m, 2H, ArH), $7.14(\mathrm{tt}, 1 \mathrm{H}, J=7.3,1.2 \mathrm{~Hz}, \operatorname{ArH}), 6.73$ (dd, $1 \mathrm{H}, J=3.1,2.1 \mathrm{~Hz}, \mathrm{CHCHN}), 1.17$ (septet, $3 \mathrm{H}, J=$ $\left.7.3 \mathrm{~Hz}, \mathrm{Si}\left(\mathrm{CH}\left(\mathrm{CH}_{3}\right)_{2}\right)_{3}\right), 0.95(\mathrm{~d}, 18, J=7.3 \mathrm{~Hz}$, $\left.\mathrm{Si}\left(\mathrm{CH}\left(\mathrm{CH}_{3}\right)_{2}\right)_{3}\right), 0.22\left(\mathrm{~s}, 9 \mathrm{H}, \mathrm{Si}\left(\mathrm{CH}_{3}\right)_{3}\right)$.

${ }^{13} \mathrm{C}$ NMR $\left(125 \mathrm{MHz}, \mathrm{CDCl}_{3}, 20{ }^{\circ} \mathrm{C}\right) \delta$ :

FTIR (Neat) $\mathrm{cm}^{-1}$ :

HRMS (ESI):

TLC (20\% EtOAc/hexanes), Rf:
$140.1,139.2,138.4,129.5,128.3,127.4,125.1,124.4$, $105.8,105.2,101.0,18.0,11.8,-0.2$.

$2948(\mathrm{~m}), 2868(\mathrm{~m}), 2141(\mathrm{w}), 1547(\mathrm{w}), 1514(\mathrm{w})$, 1488 (m), 1252 (s), 1099 (s).

calcd for $\mathrm{C}_{25} \mathrm{H}_{39} \mathrm{~N}_{2} \mathrm{Si}_{2}[\mathrm{M}+\mathrm{H}]^{+}$: 423.2646, found: 423.2656 .

$0.76\left(\mathrm{UV}, \mathrm{KMnO}_{4}\right)$. 

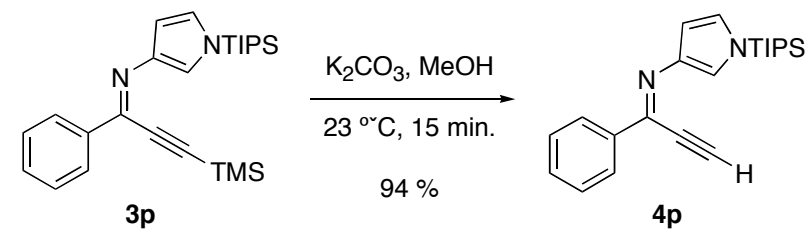

(1-Phenyl-prop-2-ynylidene)-[1-(triisopropyl-silanyl)-1H-pyrrol-3-yl]-amine (4p, Table 1, entry 16):

Anhydrous potassium carbonate $(23 \mathrm{mg}, 0.17 \mathrm{mmol}, 0.2$ equiv) was added to a solution of imine 3p (380 mg, $0.83 \mathrm{mmol}, 1$ equiv) stirred in methanol $(2.8 \mathrm{~mL})$ at $23{ }^{\circ} \mathrm{C}$. After $25 \mathrm{~min}$, the volatiles were removed under reduced pressure and the residue was purified by flash column chromatography on silica gel $(7.5 \%$ EtOAc/hexanes) to afford the alkynyl imine $4 p$ as a yellow solid (274 mg, 94\%).

${ }^{1} \mathrm{H}$ NMR $\left(500 \mathrm{MHz}, \mathrm{CDCl}_{3}, 20^{\circ} \mathrm{C}\right)$ :

${ }^{13} \mathrm{C}$ NMR $\left(125 \mathrm{MHz}, \mathrm{C}_{6} \mathrm{D}_{6}, 20^{\circ} \mathrm{C}\right)$ :

FTIR (neat):

HRMS (ESI):

TLC (30\% EtOAc-hexanes), Rf:
8.16-8.12 (m, 2H, ArH), 7.44-7.36 (m, 3H, ArH), 7.29

$(\mathrm{t}, 1 \mathrm{H}, J=1.8 \mathrm{~Hz}, \mathrm{CHN}), 7.18(\mathrm{dd}, 1 \mathrm{H}, J=3.1,1.2 \mathrm{~Hz}$, CHCHN), 6.77 (dd, 1H, J=2.7, 2.1 Hz, CHCHN), 3.69 (s, $1 \mathrm{H}, \mathrm{C} \equiv \mathrm{CH}), 1.48$ (septet, $3 \mathrm{H}, J=7.6 \mathrm{~Hz}$, $\left.\mathrm{Si}\left(\mathrm{CH}\left(\mathrm{CH}_{3}\right)_{2}\right)_{3}\right), 1.14(\mathrm{~d}, 18 \mathrm{H}, J=7.5 \mathrm{~Hz}$, $\left.\mathrm{Si}\left(\mathrm{CH}\left(\mathrm{CH}_{3}\right)_{2}\right)_{3}\right)$.

$139.8,139.7,139.4,130.1,128.8,128.0,125.4,124.6$, $107.2,86.7,80.4,18.1,12.0$.

$3296(\mathrm{~m}, \mathrm{C} \equiv \mathrm{C}-\mathrm{H}), 2947$ (s), 2868 (s), 2088 (w, C $\equiv \mathrm{C})$, 1547 (w), 1488 (s), 1098 (s).

calcd for $\mathrm{C}_{22} \mathrm{H}_{31} \mathrm{~N}_{2} \mathrm{Si}[\mathrm{M}+\mathrm{H}]$ : 351.2251, found: 351.2264 .

$0.83\left(\mathrm{UV}, \mathrm{KMnO}_{4}\right)$. 

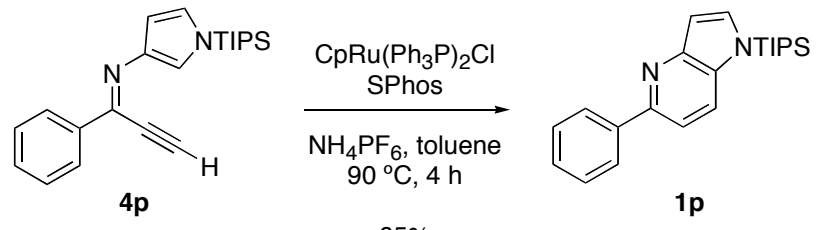

$65 \%$

\section{5-Phenyl-1-(triisopropyl-silanyl)-1H-pyrrolo[3,2-b]pyridine (1p, Table 1, entry 16):}

An oven dried pressure vessel containing a magnetic stir bar was charged with ammonium hexafluorophosphate (33 mg, $0.20 \mathrm{mmol}, 1.0$ equiv), $\mathrm{CpRu}\left(\mathrm{PPh}_{3}\right)_{2} \mathrm{Cl}$ ( $7.3 \mathrm{mg}, 0.01 \mathrm{mmol}, 0.05$ equiv) $)^{17}$ and SPhos ( $4.1 \mathrm{mg}, 0.01 \mathrm{mmol}, 0.05$ equiv) under a nitrogen atmosphere in a glove-box and the flask sealed and brought out of the glove-box. Imine 3p (70 mg, $0.20 \mathrm{mmol}, 1$ equiv) and toluene $(1.0 \mathrm{~mL})$ were subsequently added via syringe. The flask was flushed with argon, sealed, stirred, and placed in an oil bath at $90^{\circ} \mathrm{C}$. After $4 \mathrm{~h}$, the reaction vessel was allowed to cool to ambient temperature and was diluted with dichloromethane $(3 \mathrm{~mL})$. This solution was concentrated under reduced pressure and the residue was purified by flash column chromatography on basic alumina (15 $\%$ EtoAc/Hexanes) to afford the azaindole 1p as a pale brown solid (46 mg, 65\%).

${ }^{1} \mathrm{H} \mathrm{NMR}\left(500 \mathrm{MHz}, \mathrm{CDCl}_{3}, 20^{\circ} \mathrm{C}\right)$ :

${ }^{13} \mathrm{C}$ NMR $\left(125 \mathrm{MHz}, \mathrm{CDCl}_{3}, 20^{\circ} \mathrm{C}\right)$ :

FTIR (neat):

HRMS (ESI):

TLC (20\% EtOAc-hexanes), Rf:
8.46-8.42 (m, 2H, ArH), $7.58(\mathrm{dd}, 1 \mathrm{H}, J=8.7,0.9 \mathrm{~Hz}$, ArH), 7.53 (d, 1H, $J=8.5 \mathrm{~Hz}, \operatorname{ArH}), 7.39-7.34$ (m, 2H, ArH), 7.22 (tt, $1 \mathrm{H}, J=7.3,1.2 \mathrm{~Hz}, \operatorname{ArH}), 7.14(\mathrm{~d}, 1 \mathrm{H}, J$ $=3.4 \mathrm{~Hz}, \mathrm{NCHCHC}), 7.11(\mathrm{dd}, 1 \mathrm{H}, J=3.4,0.6 \mathrm{~Hz}$, NCHCHC), 1.32 (septet, $3 \mathrm{H}, J=7.6 \mathrm{~Hz}$, $\left.\mathrm{Si}\left(\mathrm{CH}\left(\mathrm{CH}_{3}\right)_{2}\right)_{3}\right), 0.93(\mathrm{~d}, 18 \mathrm{H}, J=7.6 \mathrm{~Hz}$, $\left.\mathrm{Si}\left(\mathrm{CH}\left(\mathrm{CH}_{3}\right)_{2}\right)_{3}\right)$.

$151.9,151.4,141.8,135.5,133.3,129.2,128.5,127.8$, 121.3, 114.5, 107.8, 18.3, 13.0.

3062 (w), 2948 (m), $2868(\mathrm{~m}), 1561(\mathrm{w}), 1510(\mathrm{w})$, $1465(\mathrm{~m}), 1407(\mathrm{~s}), 1138(\mathrm{~m})$.

calcd for $\mathrm{C}_{22} \mathrm{H}_{31} \mathrm{~N}_{2} \mathrm{Si}[\mathrm{M}+\mathrm{H}]$ : 351.2251 , found: 351.2245 .

$0.58\left(\mathrm{UV}, \mathrm{KMnO}_{4}\right)$.

\footnotetext{
${ }^{17}$ Use of higher catalyst loadings led to more $N$-desilylation of the product without improvement in yield of $\mathbf{1 p .}$
} 


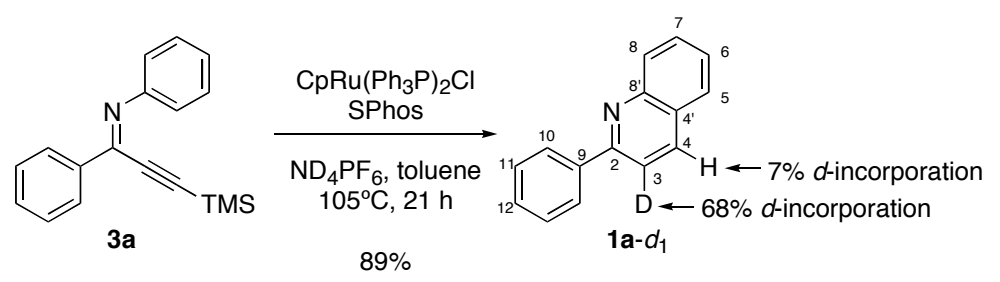

\section{3-Deutero-2-phenylquinoline (1a- $d_{1}$, Scheme S1):}

An oven dried pressure vessel containing a magnetic stir bar was charged with ammonium hexafluorophosphate $\left(d_{4}\right)\left(42 \mathrm{mg}, 0.25 \mathrm{mmol}, 1.0\right.$ equiv), $\mathrm{CpRu}\left(\mathrm{PPh}_{3}\right)_{2} \mathrm{Cl}(18 \mathrm{mg}, 0.025 \mathrm{mmol}, 0.10$ equiv) and SPhos (10 mg, $0.025 \mathrm{mmol}, 0.10$ equiv) under a nitrogen atmosphere in a glove-box and the flask sealed and brought out of the glove-box. Freshly distilled benzene $(2 \times 3 \mathrm{~mL})$ was added to the pressure vessel and subsequently removed in vacuo. Imine 3a (70 mg, $0.25 \mathrm{mmol}, 1$ equiv) and toluene $(1.3 \mathrm{~mL})$ were subsequently added via syringe. The flask was flushed with argon, sealed, stirred, and placed in an oil bath at $105^{\circ} \mathrm{C}$. After $21 \mathrm{~h}$, the reaction vessel was allowed to cool to ambient temperature and was diluted with dichloromethane $(5 \mathrm{~mL})$. This solution was concentrated under reduced pressure and the residue was purified by flash column chromatography on silica gel ( 5 $\%$ EtoAc/Hexanes) to afford the quinoline $1 \mathbf{a}-d_{1}$ as a pale yellow solid $(46 \mathrm{mg}, 89 \%) .{ }^{1} \mathrm{H}$ and ${ }^{13} \mathrm{C}$ NMR analysis indicates the presence of $\mathbf{1 a}-\mathrm{C} 3-\mathrm{H}^{2}, \mathbf{1 a}-\mathrm{C} 4-\mathrm{H}^{2}$, and $\mathbf{1 a}$ in a ratio of $68: 7: 25$.

${ }^{1} \mathrm{H}$ NMR (500 MHz, $\mathrm{CDCl}_{3}, 20{ }^{\circ} \mathrm{C}$; resonances corresponding to the $\mathrm{C} 3-\mathrm{H}^{2}$ noted by *) $\delta: 8.25(\mathrm{~d}, 1 \mathrm{H}$, $\left.J=8.2 \mathrm{~Hz}, \mathrm{C} 4-\mathrm{H}^{*}\right), 8.24(\mathrm{~s}, 1 \mathrm{H}, \mathrm{C} 4-\mathrm{H}), 8.21-8.16(\mathrm{~m}$, $\left.6 \mathrm{H}, \mathrm{C} 8-\mathrm{H}, \mathrm{C} 8-\mathrm{H}^{*}, \mathrm{C} 10-\mathrm{H}, \mathrm{C} 10-\mathrm{H}^{*}\right), 7.91(\mathrm{~d}, 1 \mathrm{H}, J=8.6$ $\mathrm{Hz}, \mathrm{C} 3-\mathrm{H}), 7.85$ (dd, $1 \mathrm{H}, J=8.2,1.2 \mathrm{~Hz}, \mathrm{C} 5-\mathrm{H}, \mathrm{C} 5-\mathrm{H}^{*}$ ), 7.74 (ddd, $\left.1 \mathrm{H}, J=8.2,6.7,1.2 \mathrm{~Hz}, \mathrm{C} 7-\mathrm{H}, \mathrm{C} 7-\mathrm{H}^{*}\right), 7.57-$ 7.52 (m, 6H, C6-H*, C6-H, C11-H, C11-H*), 7.48 (tt, $\left.1 \mathrm{H}, J=7.6,1.2 \mathrm{~Hz}, \mathrm{C} 12-\mathrm{H}, \mathrm{C} 12-\mathrm{H}^{*}\right)$.

${ }^{13} \mathrm{C}$ NMR (125 MHz, $\mathrm{CDCl}_{3}, 20{ }^{\circ} \mathrm{C}$; resonances corresponding to the $\mathrm{C} 3-\mathrm{H}^{2}$ noted by *) $\delta: 157.5(\mathrm{C} 2)$, $157.4(\mathrm{C} 2 *), 148.5(\mathrm{C} 8 '), 148.4(\mathrm{C} 8 *), 139.9$ (C9), $139.8(\mathrm{C} 9 *), 136.9(\mathrm{C} 4 *), 136.8(\mathrm{C} 4), 129.9(\mathrm{C} 7, \mathrm{C} 7 *)$, $129.8(\mathrm{C} 8, \mathrm{C} 8 *), 129.5(\mathrm{C} 12, \mathrm{C} 12 *), 129.0\left(\mathrm{C} 11, \mathrm{C} 11^{*}\right)$, 127.7 (C10, C10*), $127.6\left(\mathrm{C} 5, \mathrm{C} 5^{*}\right), 127.3\left(\left(4^{\prime}, 4^{\prime *}\right)\right.$, $126.4\left(6,6^{*}\right), 119.1(\mathrm{C} 3), 119.0\left(\mathrm{t}, J=21 \mathrm{~Hz}, \mathrm{C} 3^{*}\right)$.

FTIR $\left(\mathrm{CDCl}_{3}\right) \mathrm{cm}^{-1}$ :

HRMS (ESI):

TLC (20\% EtOAc-hexanes), Rf:
3056 (m), 2918 (w), 2248 (w, C-D), 1962 (w), 1616 (m), 1589 (s), 1552 (s), 1589 (s), 1490 (s).

calcd for $\mathrm{C}_{15} \mathrm{H}_{11} \mathrm{DN}[\mathrm{M}+\mathrm{H}]: 207.1027$, found: 207.1036.

0.51 (UV, CAM). 


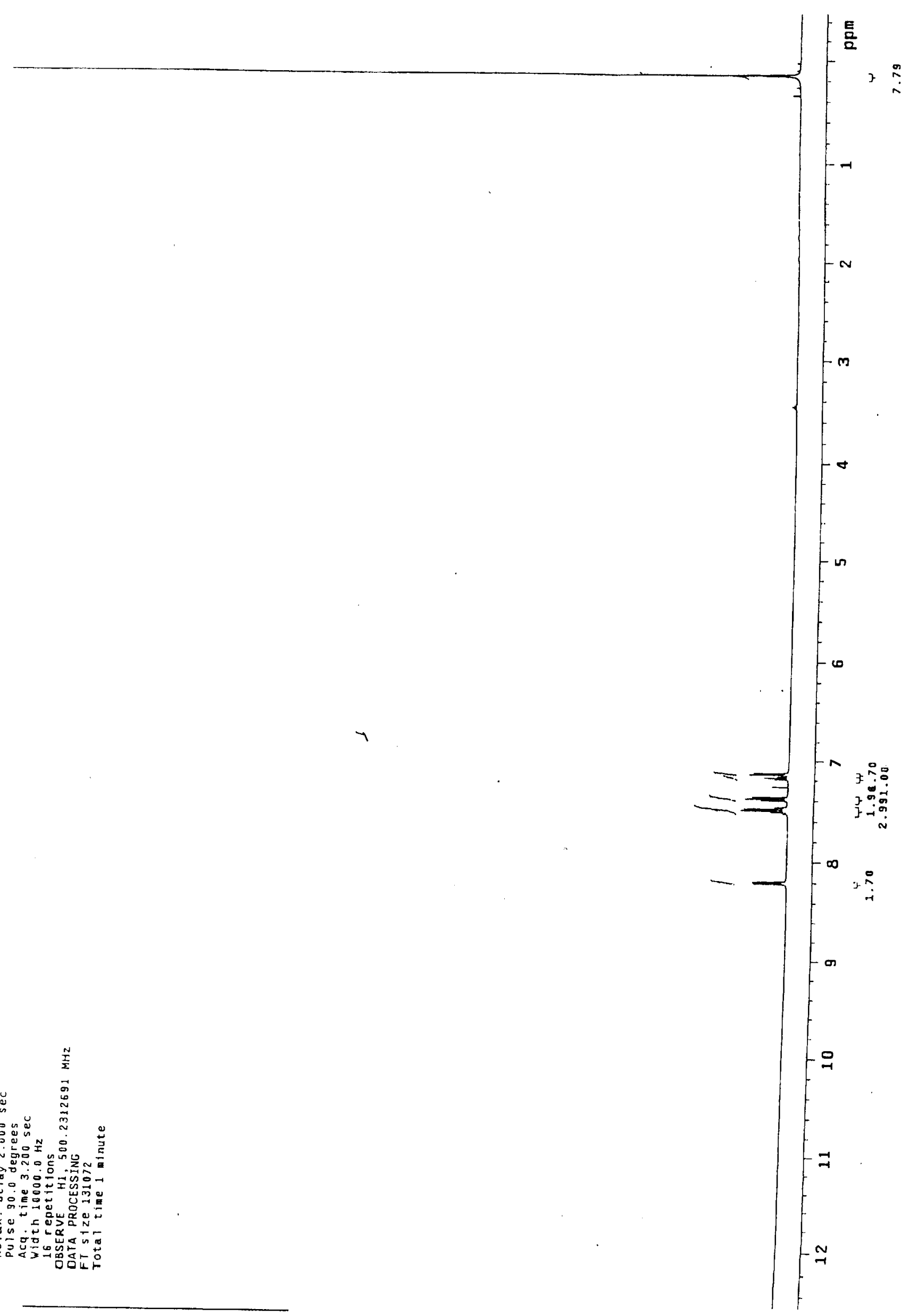

$N$-Phenyl-2-phenyl-4-trimethylsilyl-1-azabut-1-en-3-yne 
$8<6.9 \angle-$

OEZ $980, \angle L$

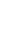

E? $2 \cdot 0=$
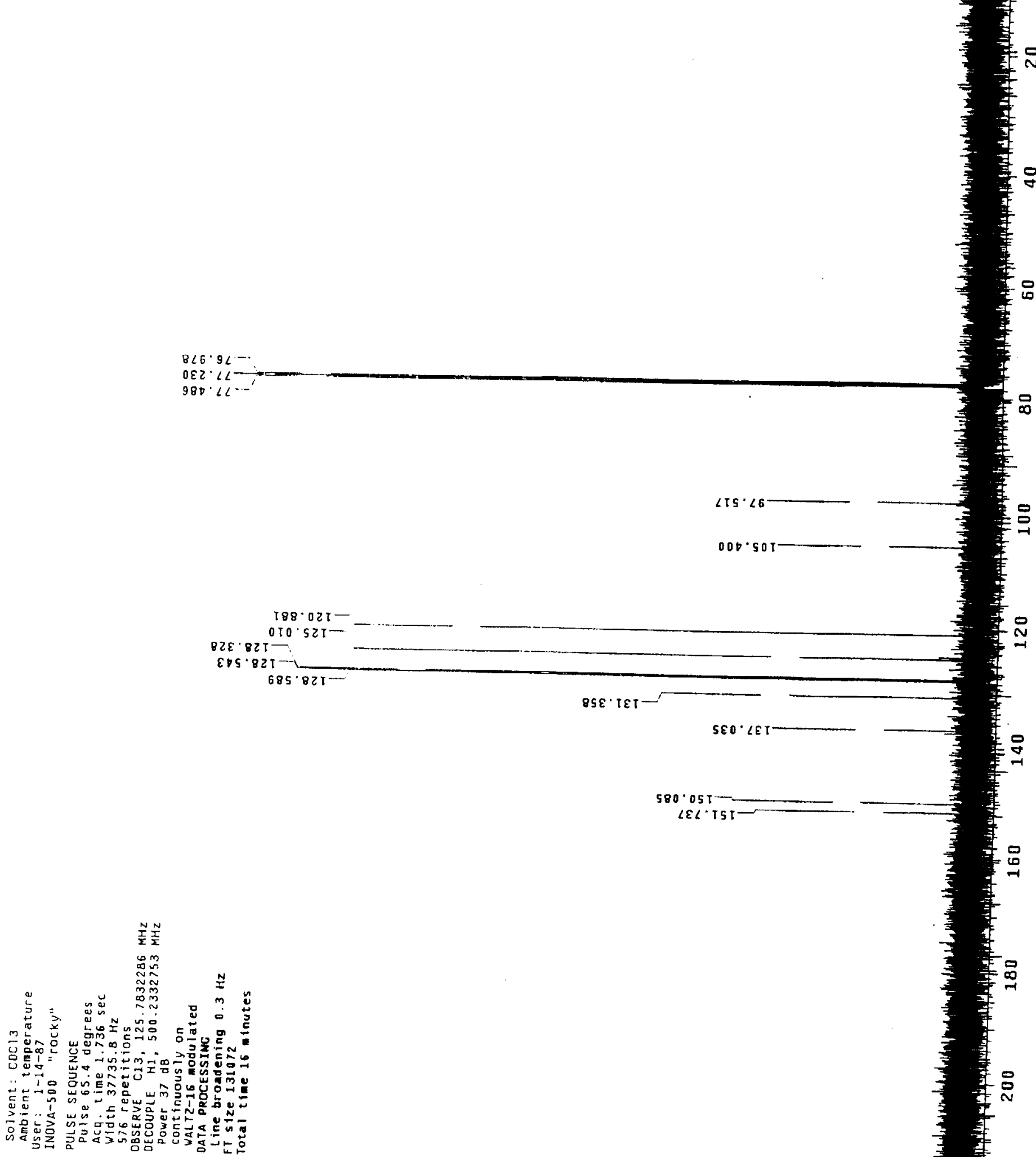

$B 2 E \cdot 82 \mathrm{~T}$

188.021

$\varepsilon \nabla 5 \cdot 82 \tau$

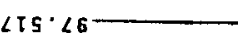

$000.501-$

$\angle \varepsilon L \cdot T 5 T$

$0^{\circ} \angle E T \longrightarrow$
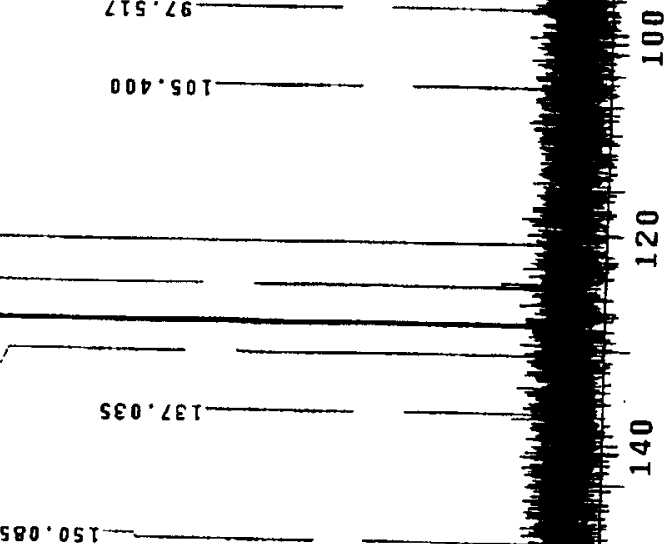


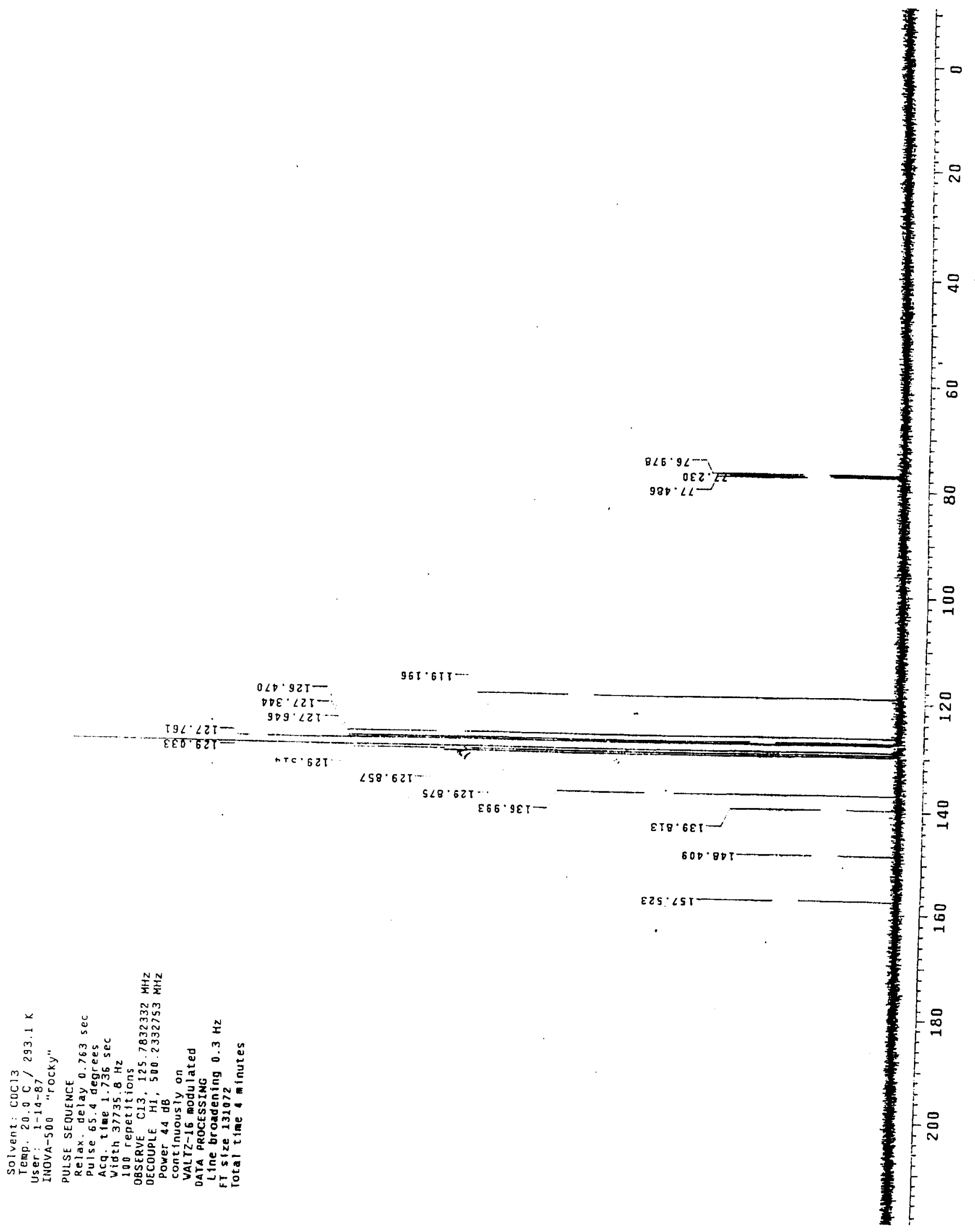

2-Phenylquinoline 


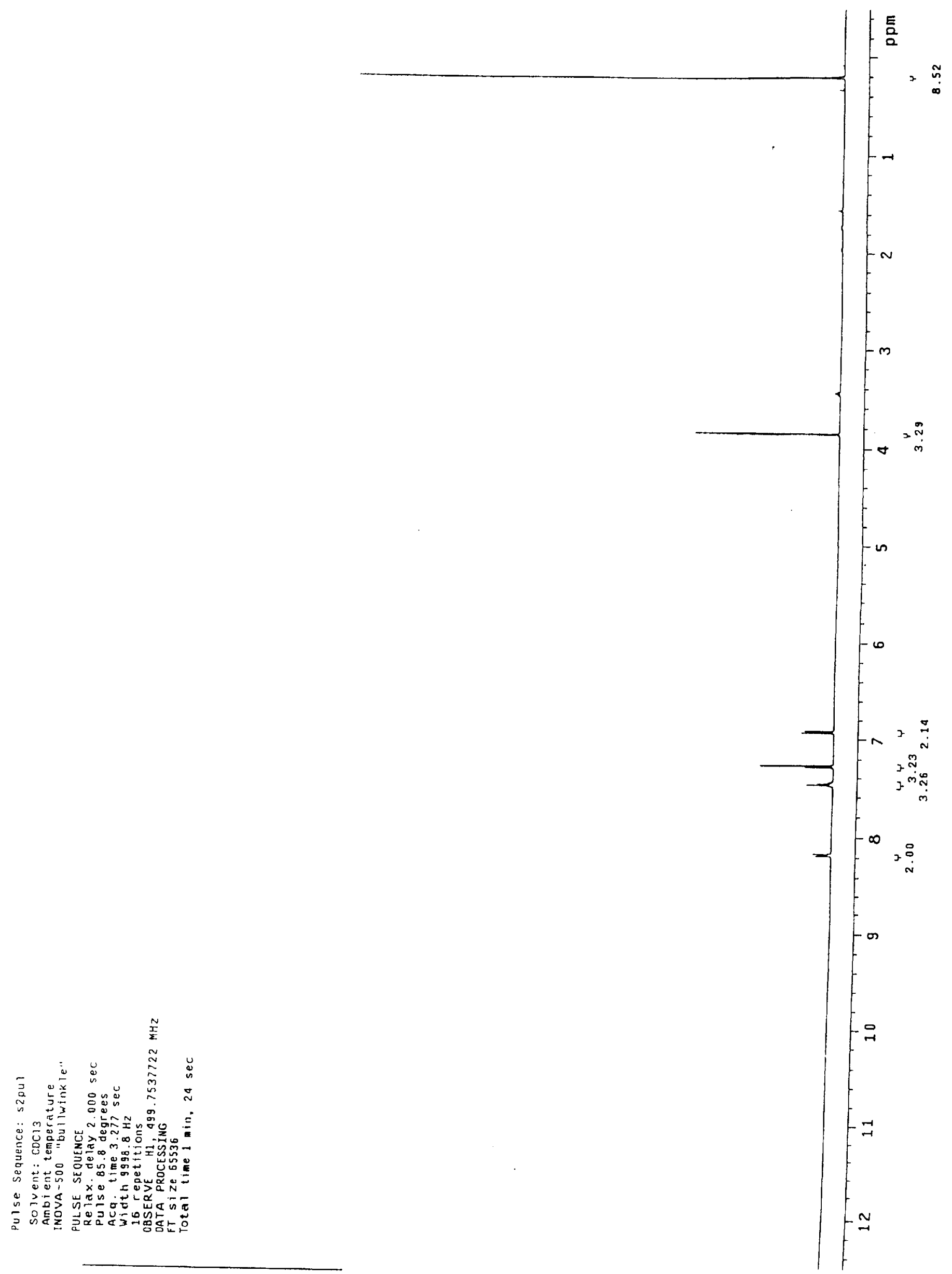

$N$-(4-Methoxyphenyl)-2-phenyl-4-trimethylsilyl-1-azabut-1-en-3-yne 


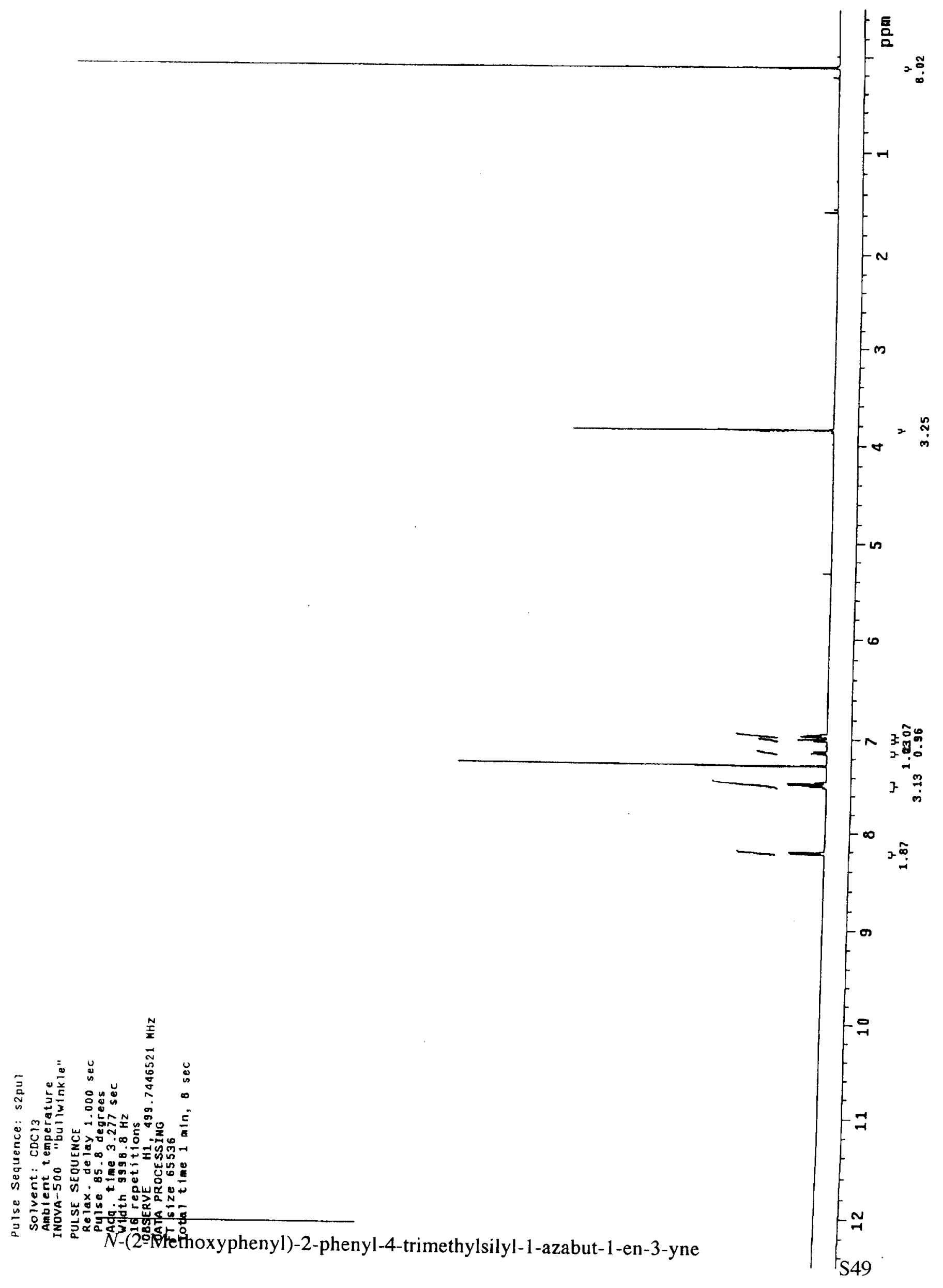




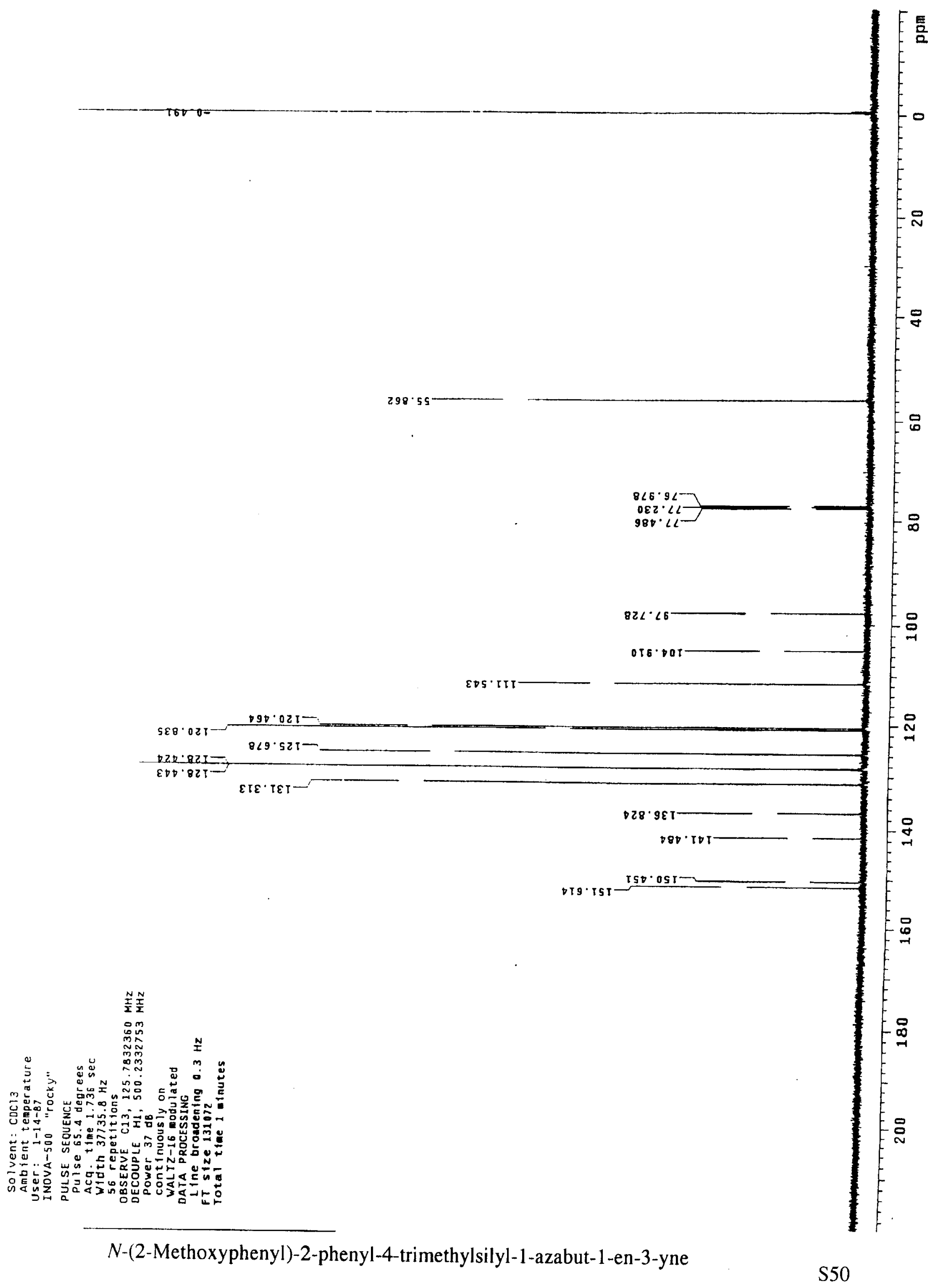




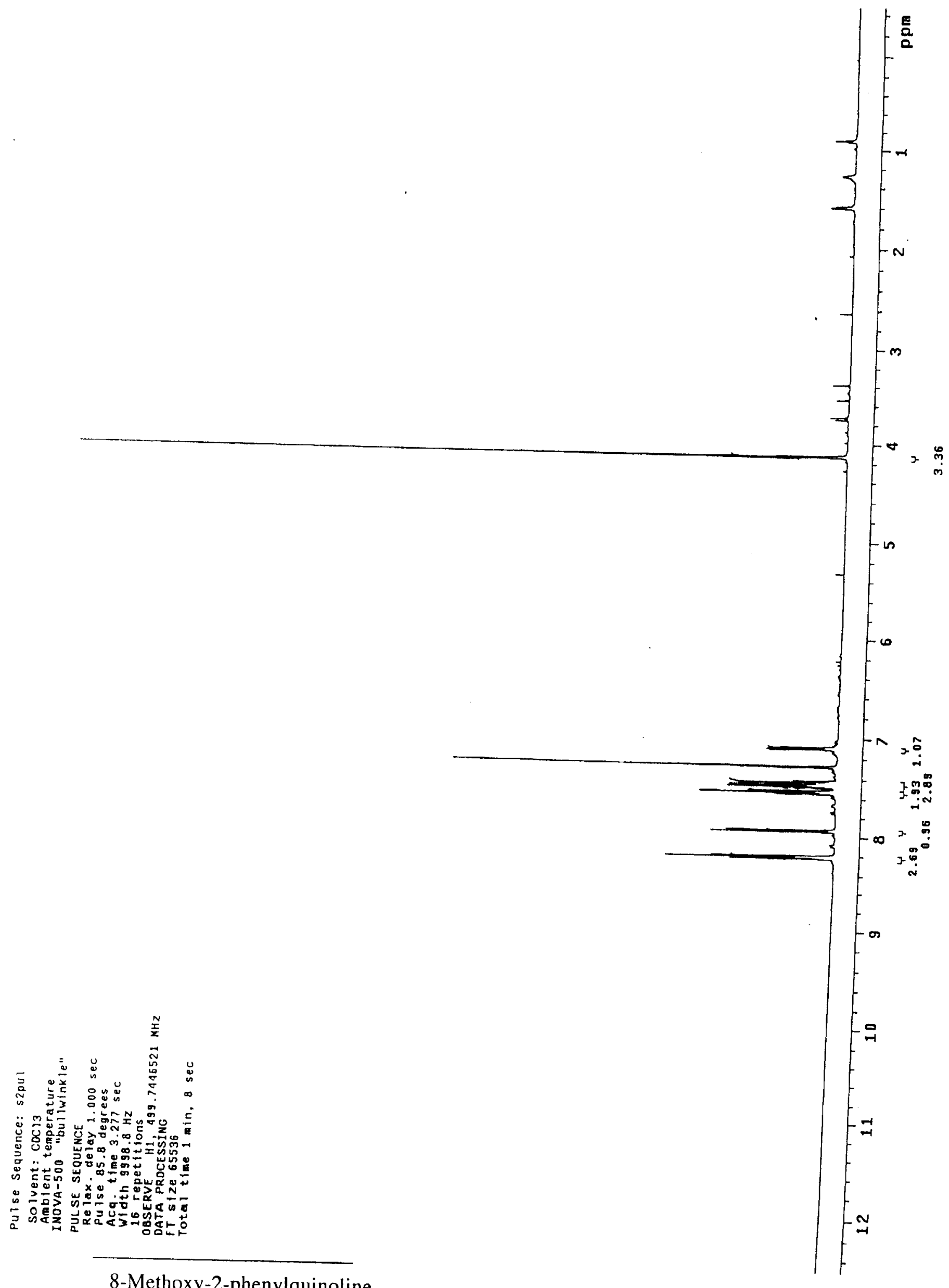

8-Methoxy-2-phenylquinoline 


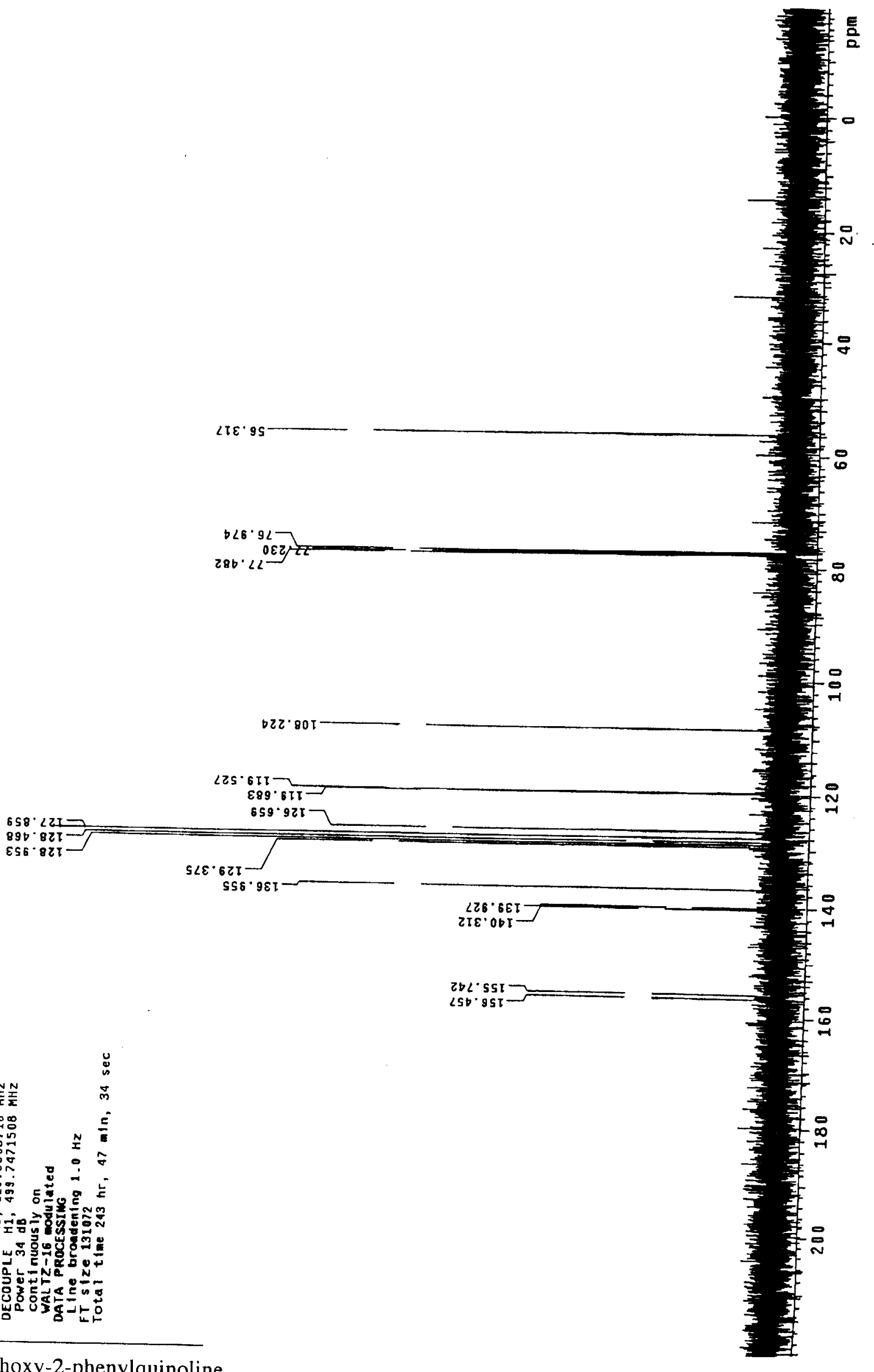

8-Methoxy-2-phenylquinoline 


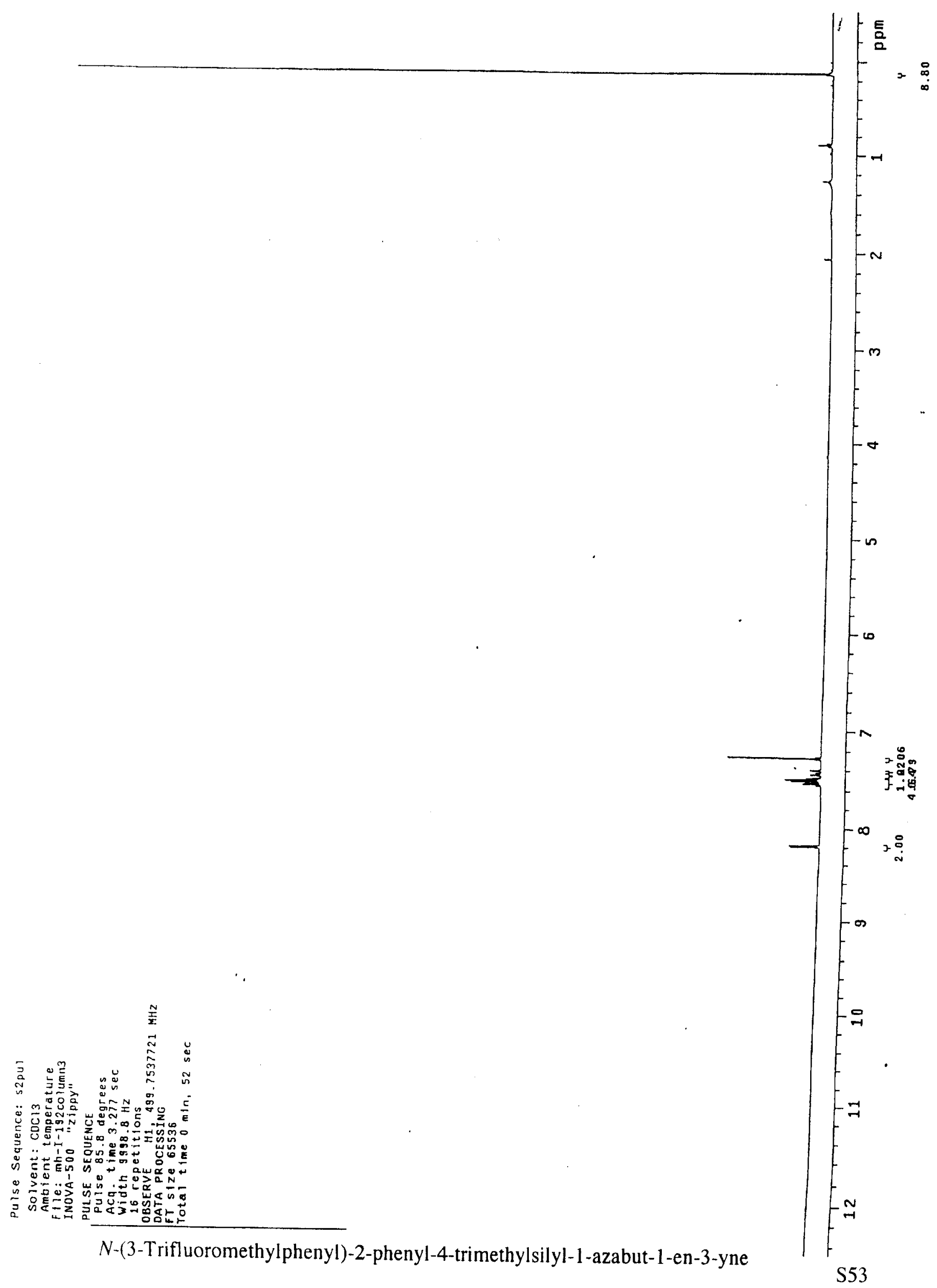




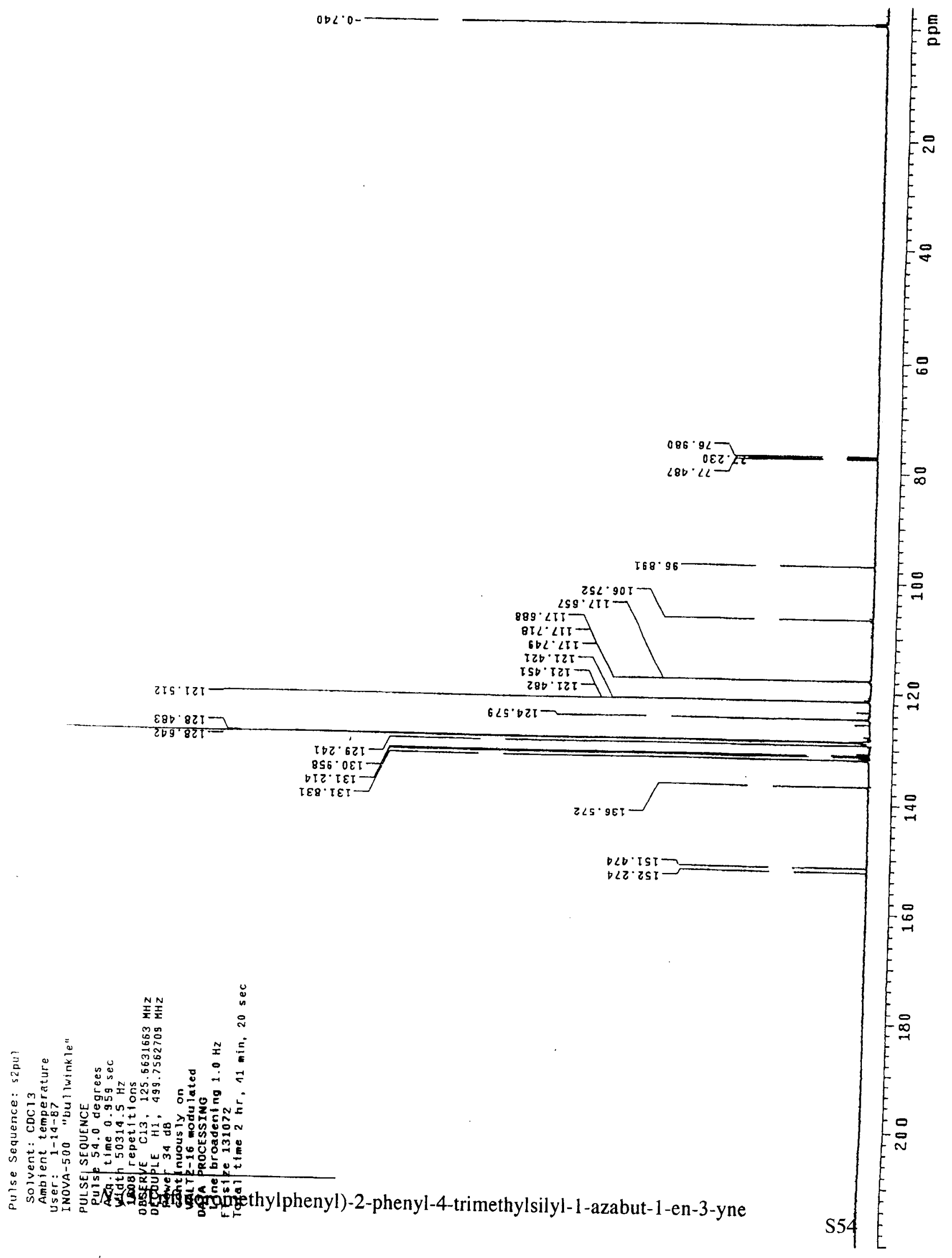




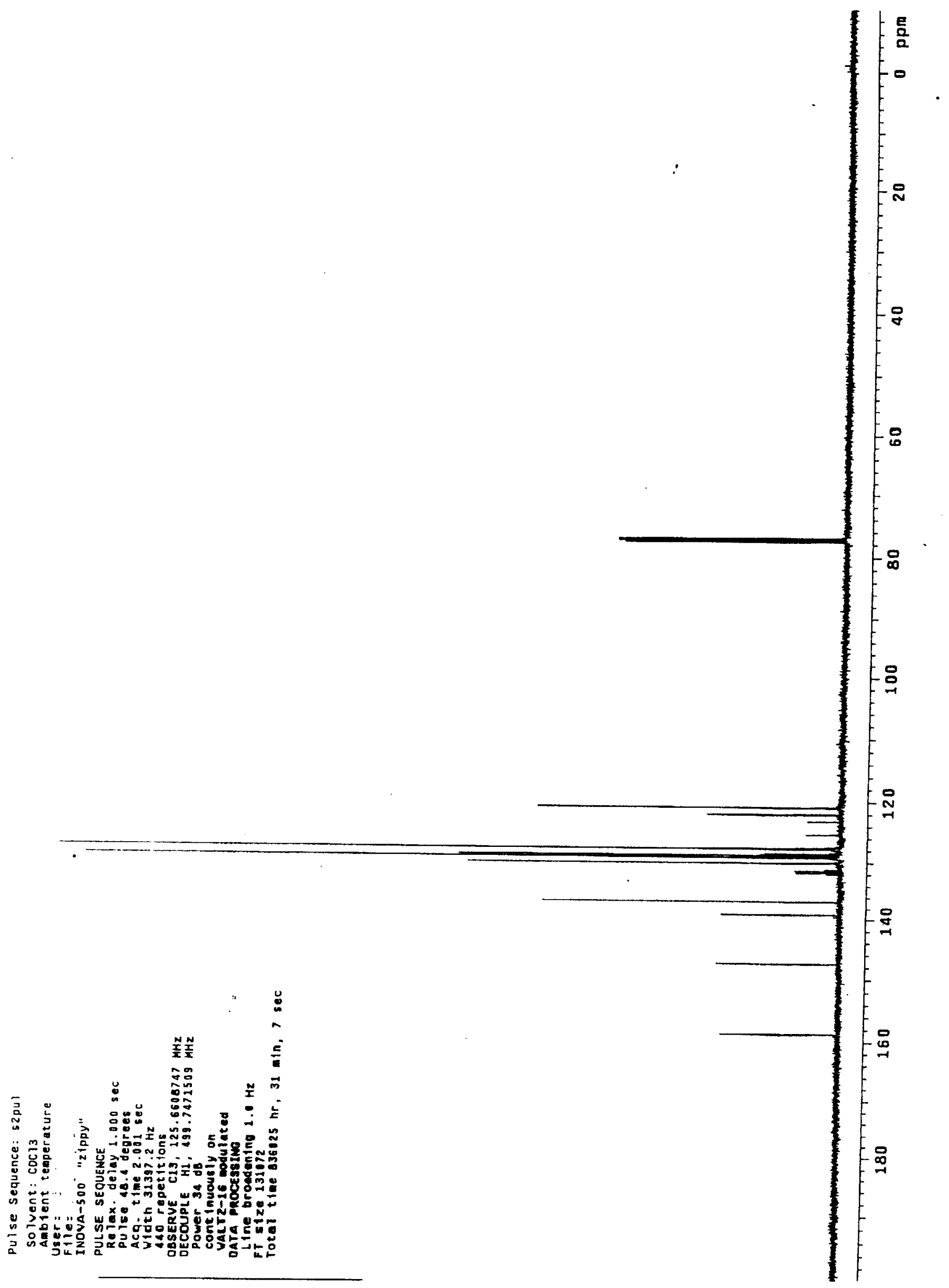

2-Phenyl-7-trifluoromethyl-quinoline 


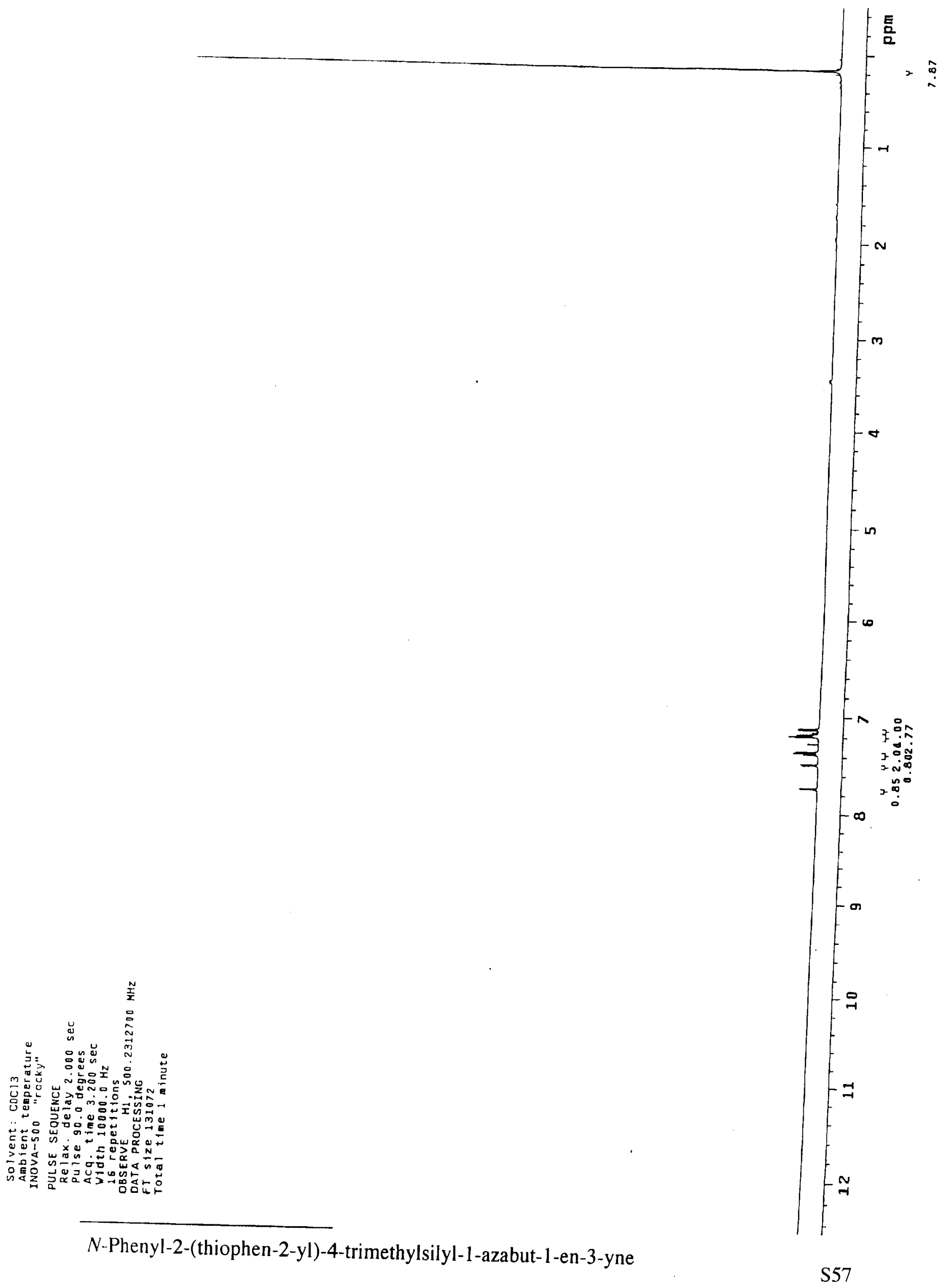




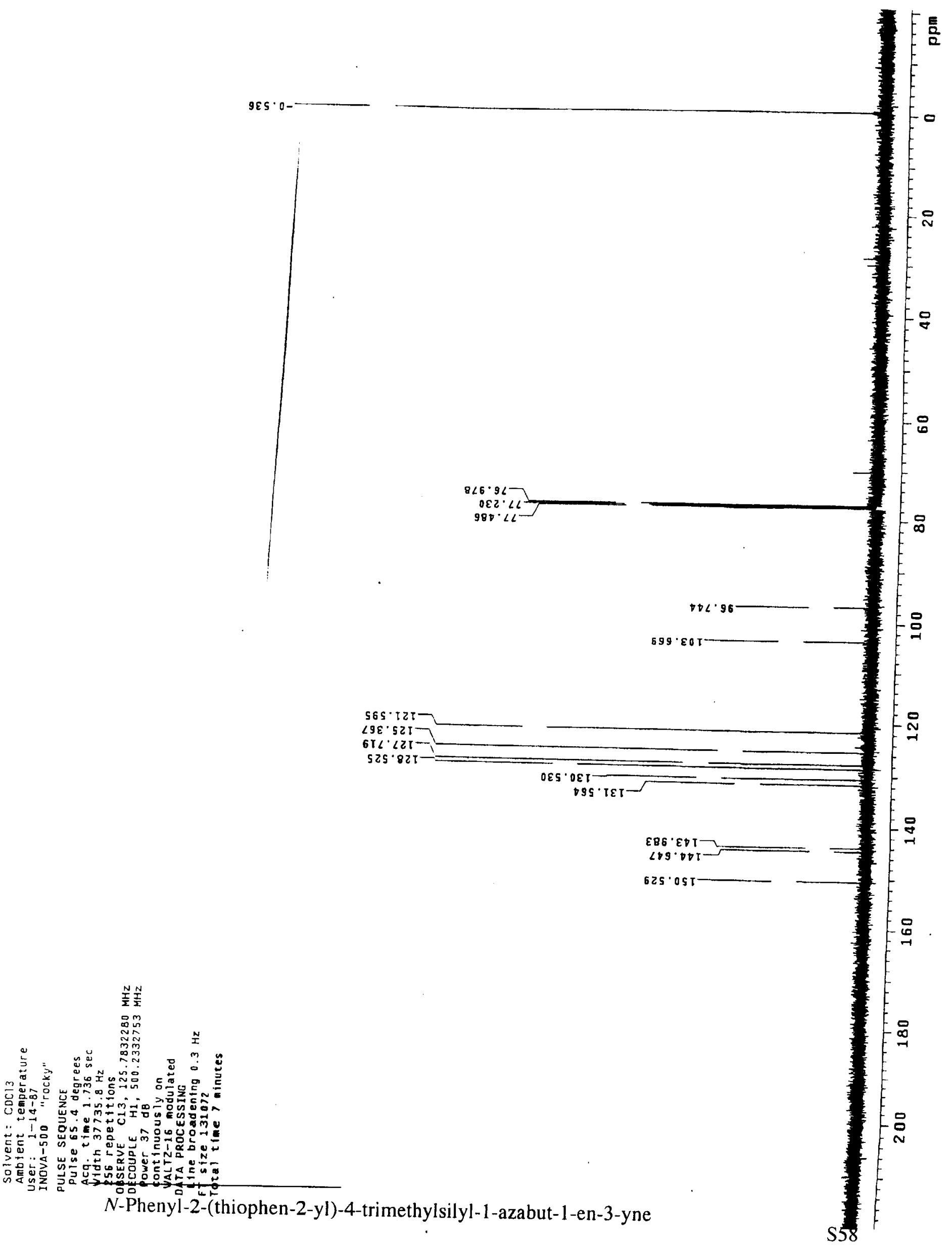




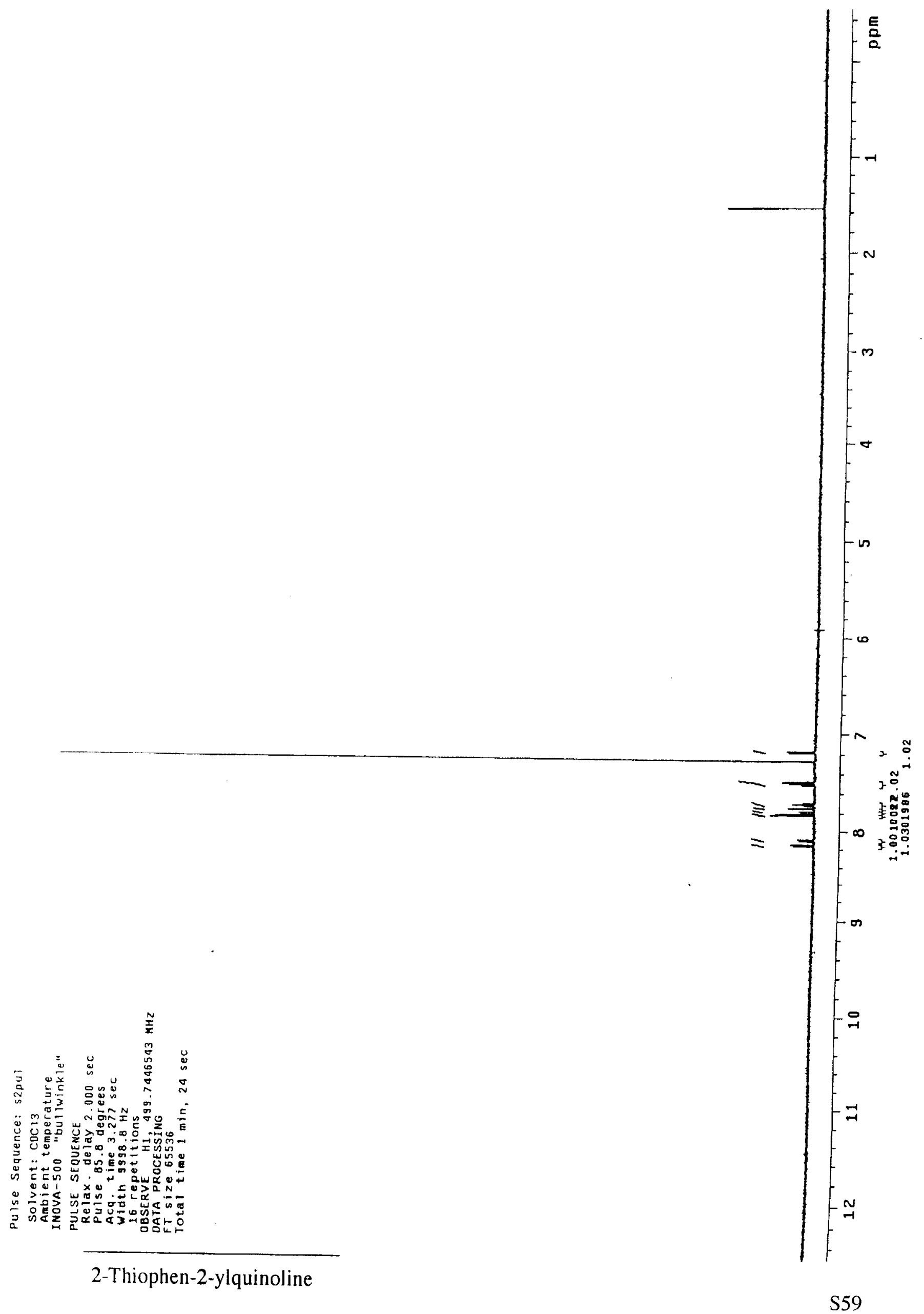




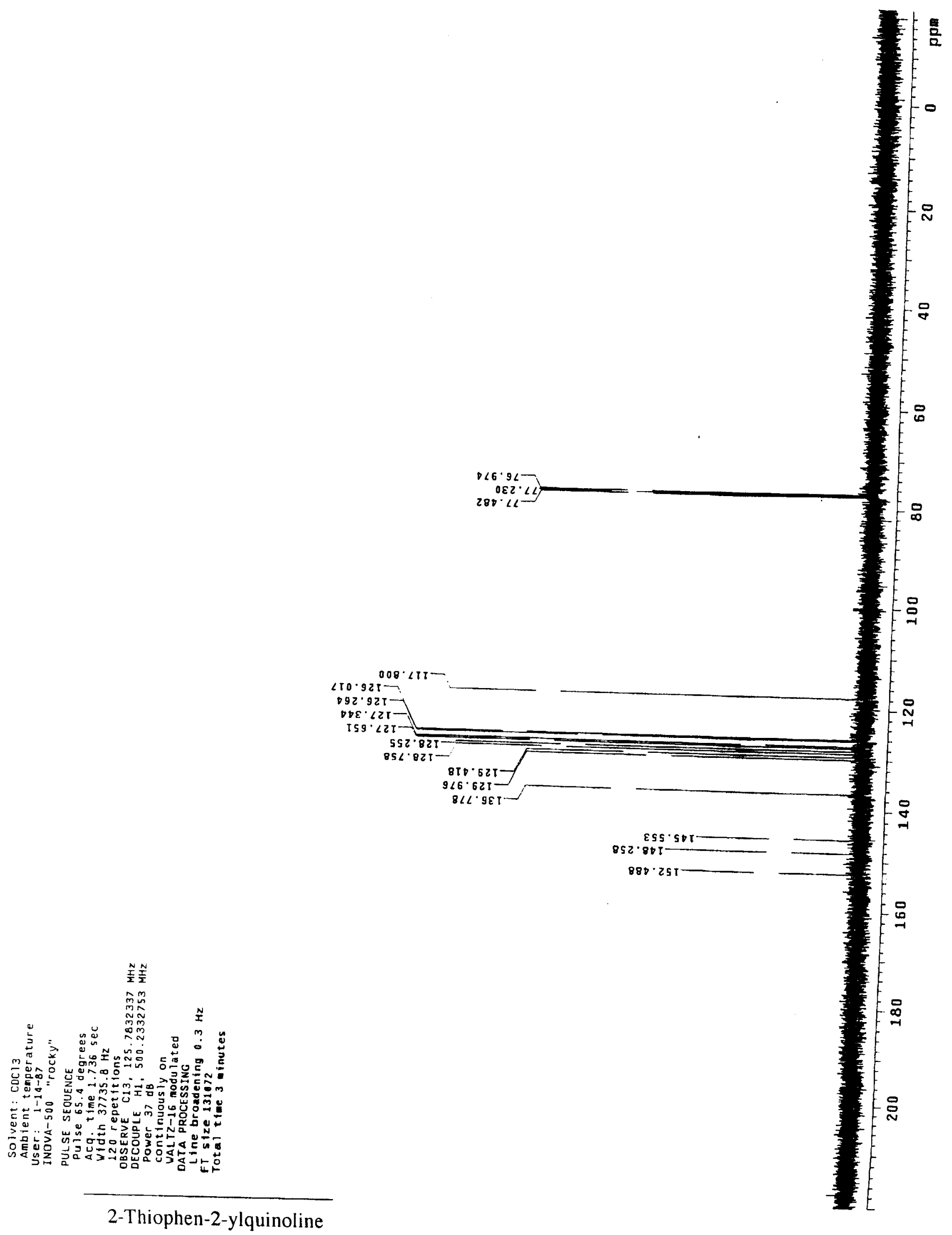




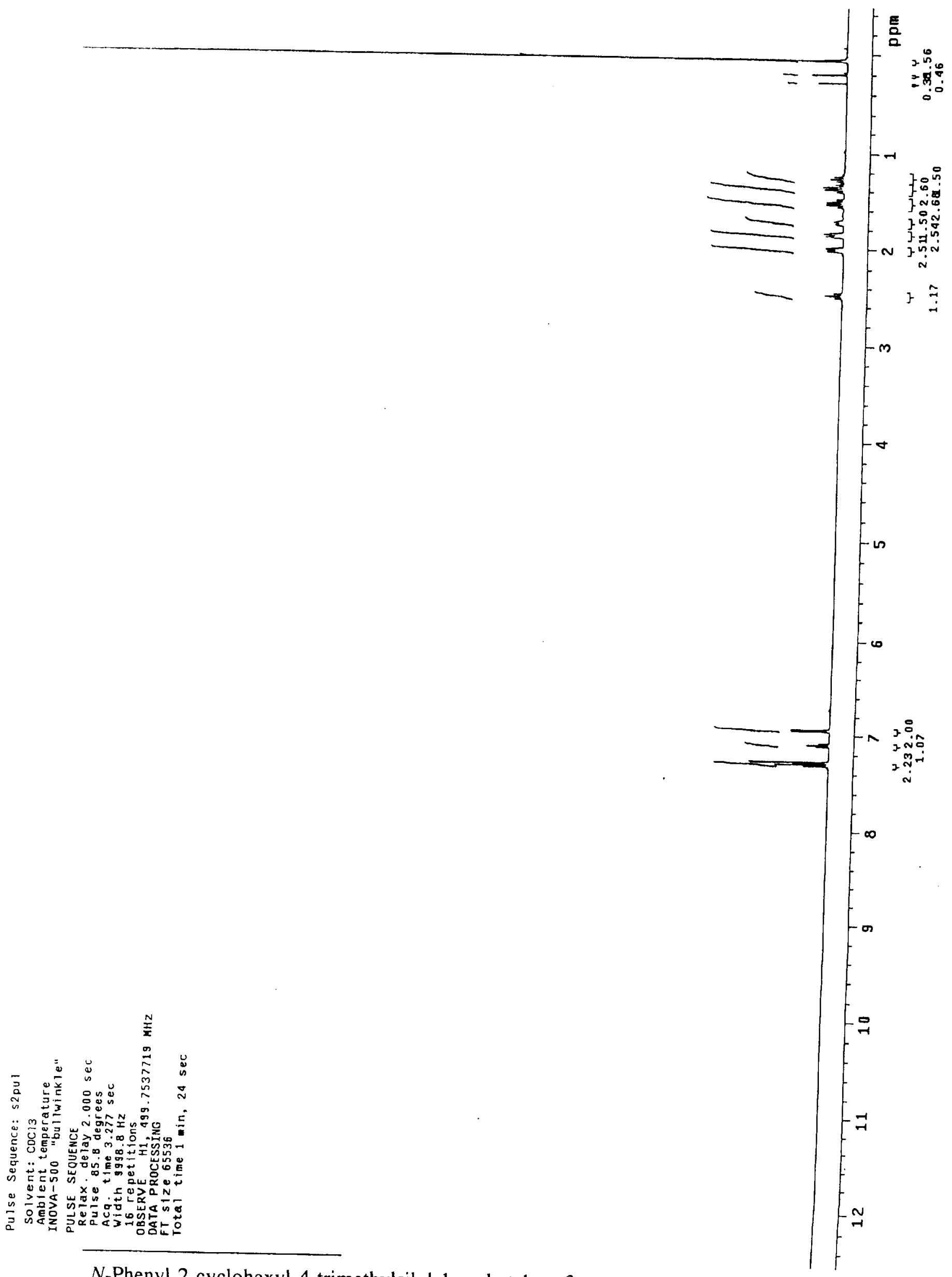

$N$-Phenyl-2-cyclohexyl-4-trimethylsilyl-1-azabut-1-en-3-yne 


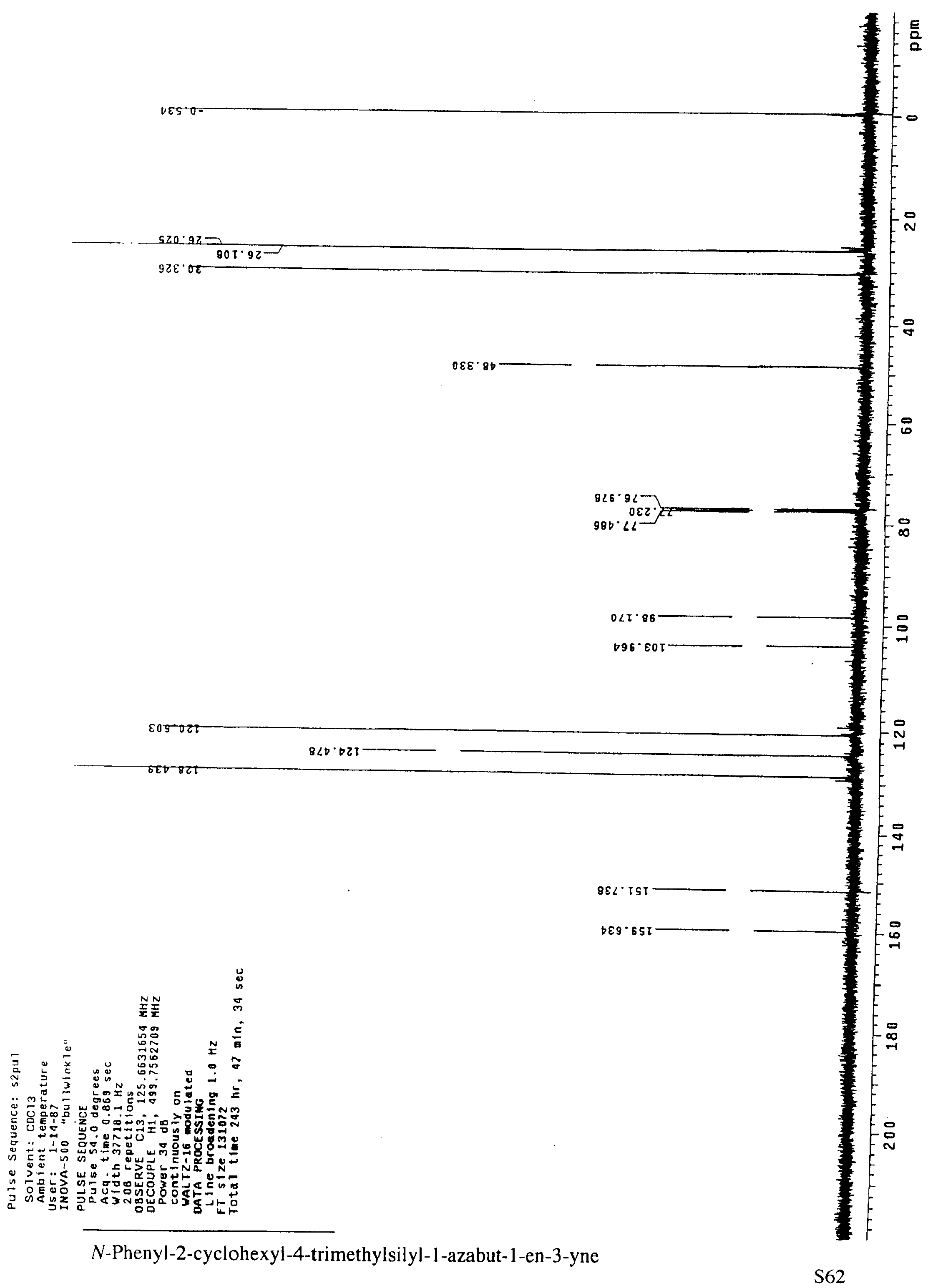




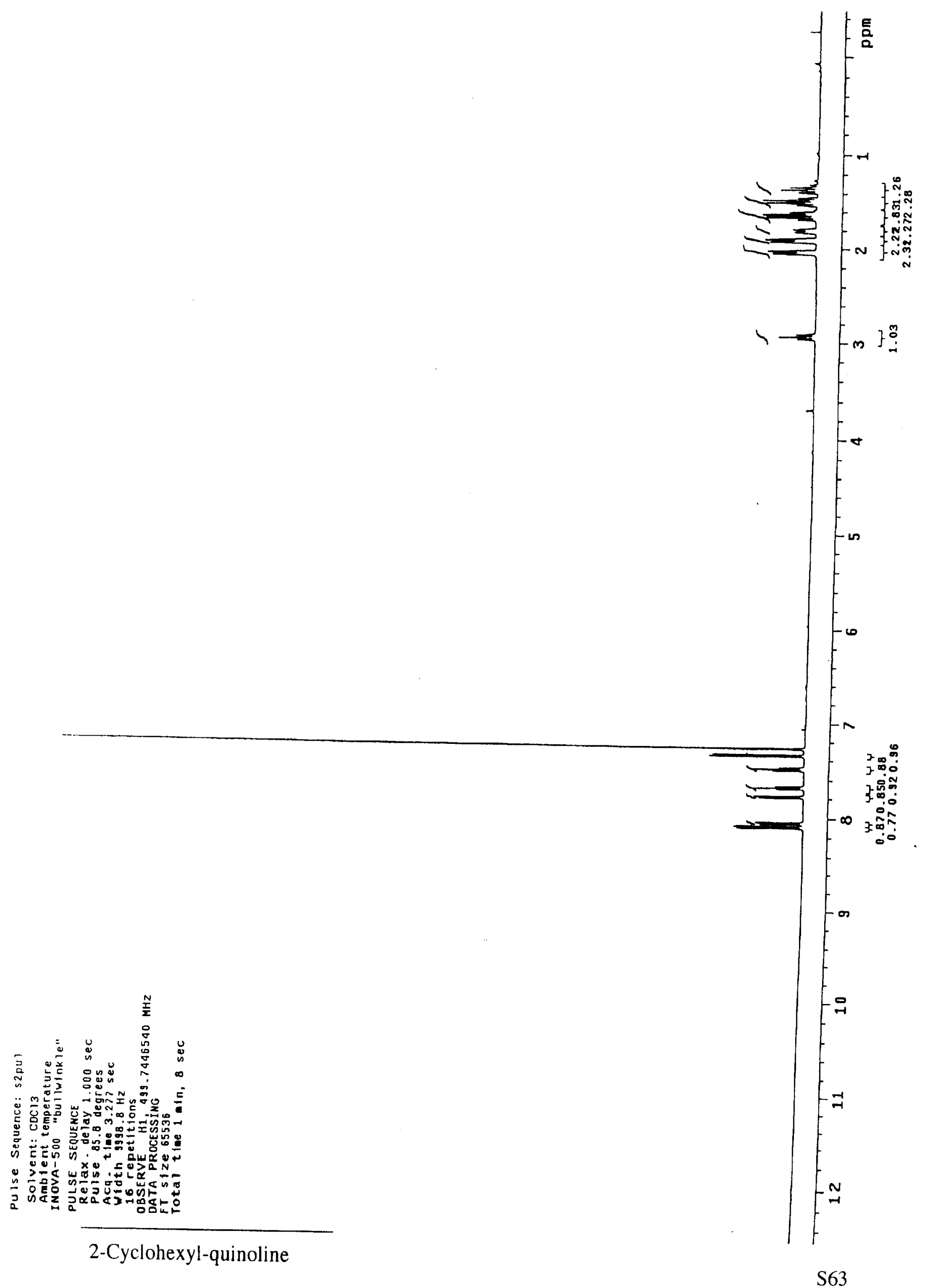




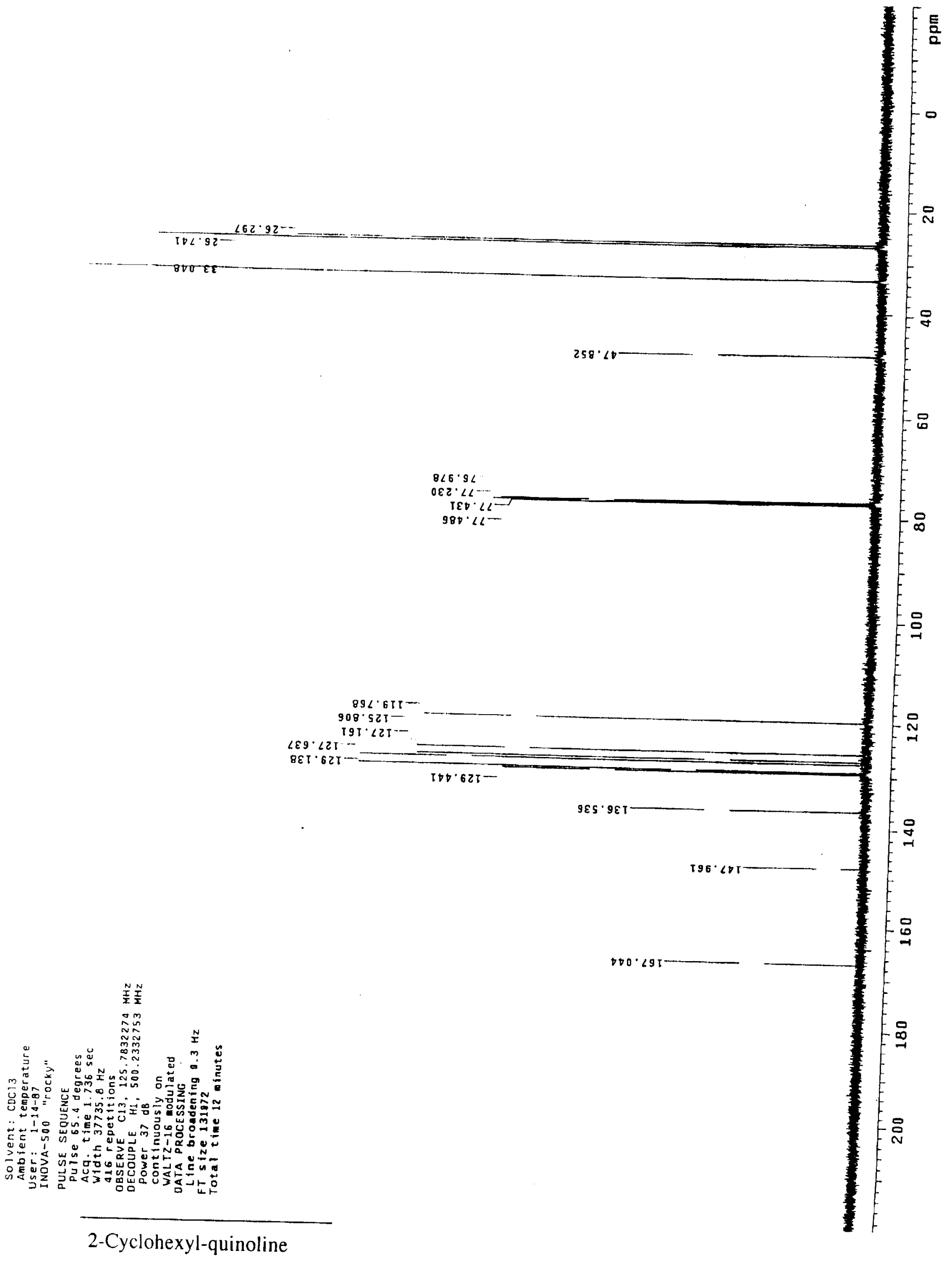




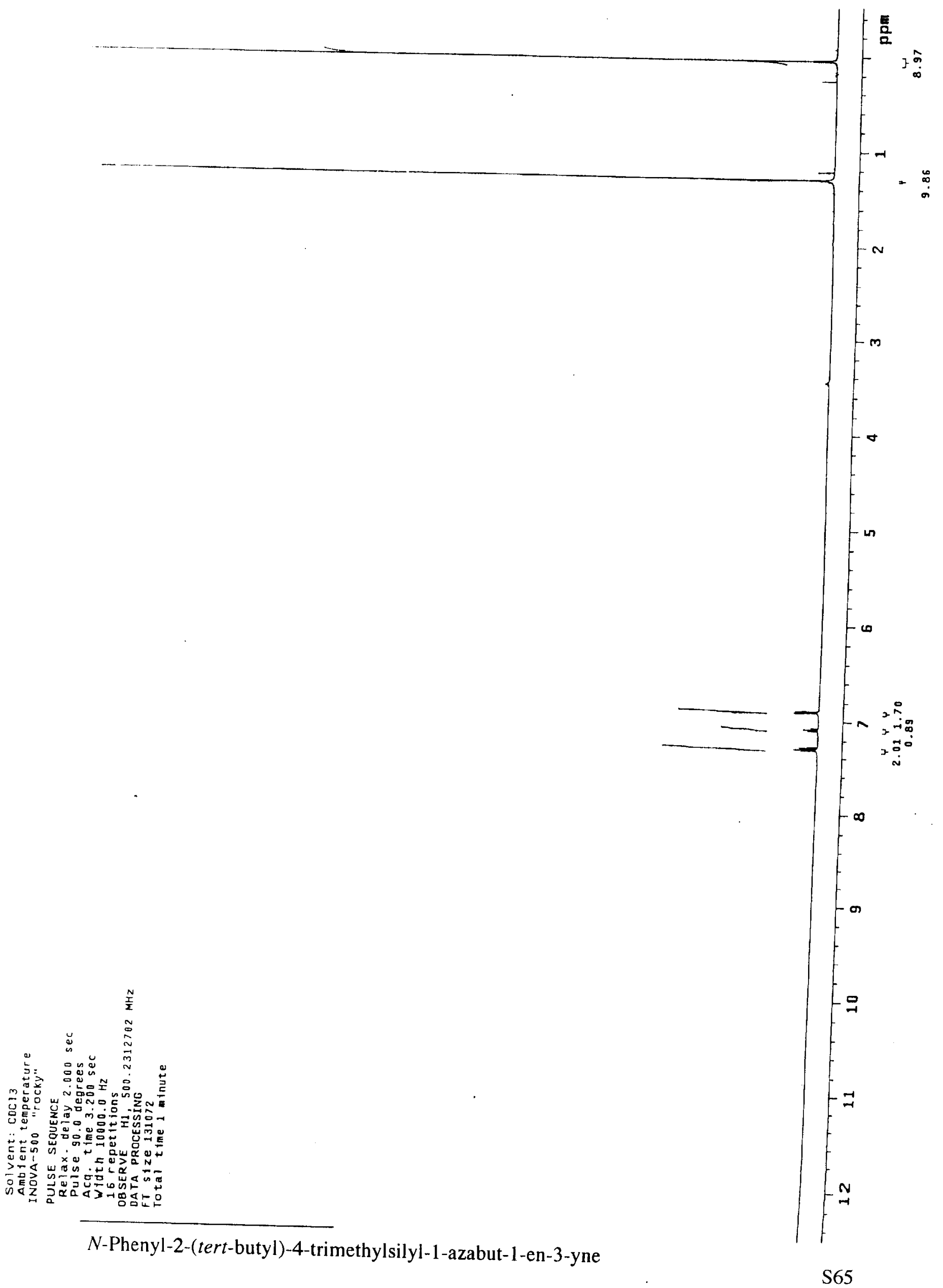




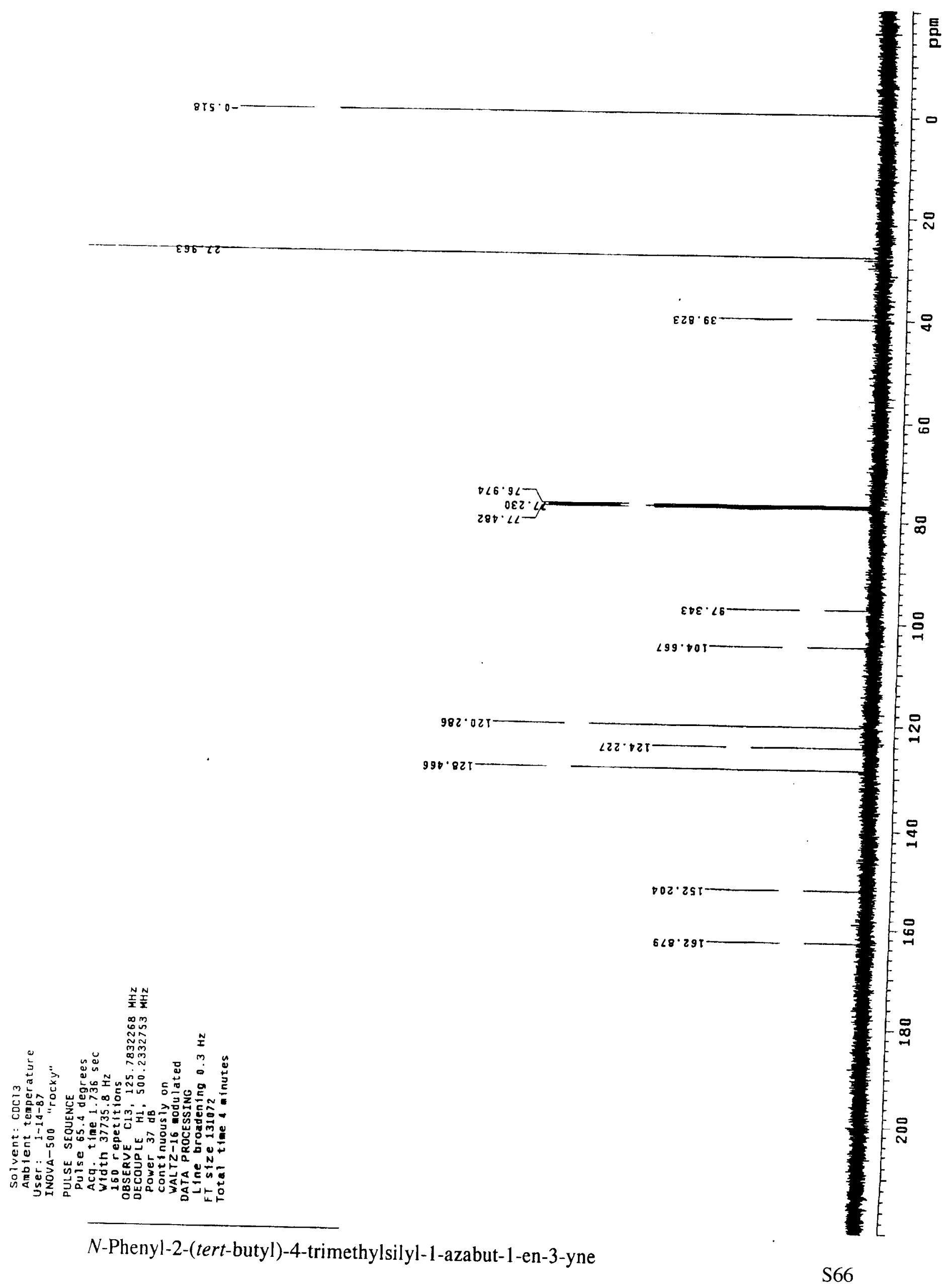




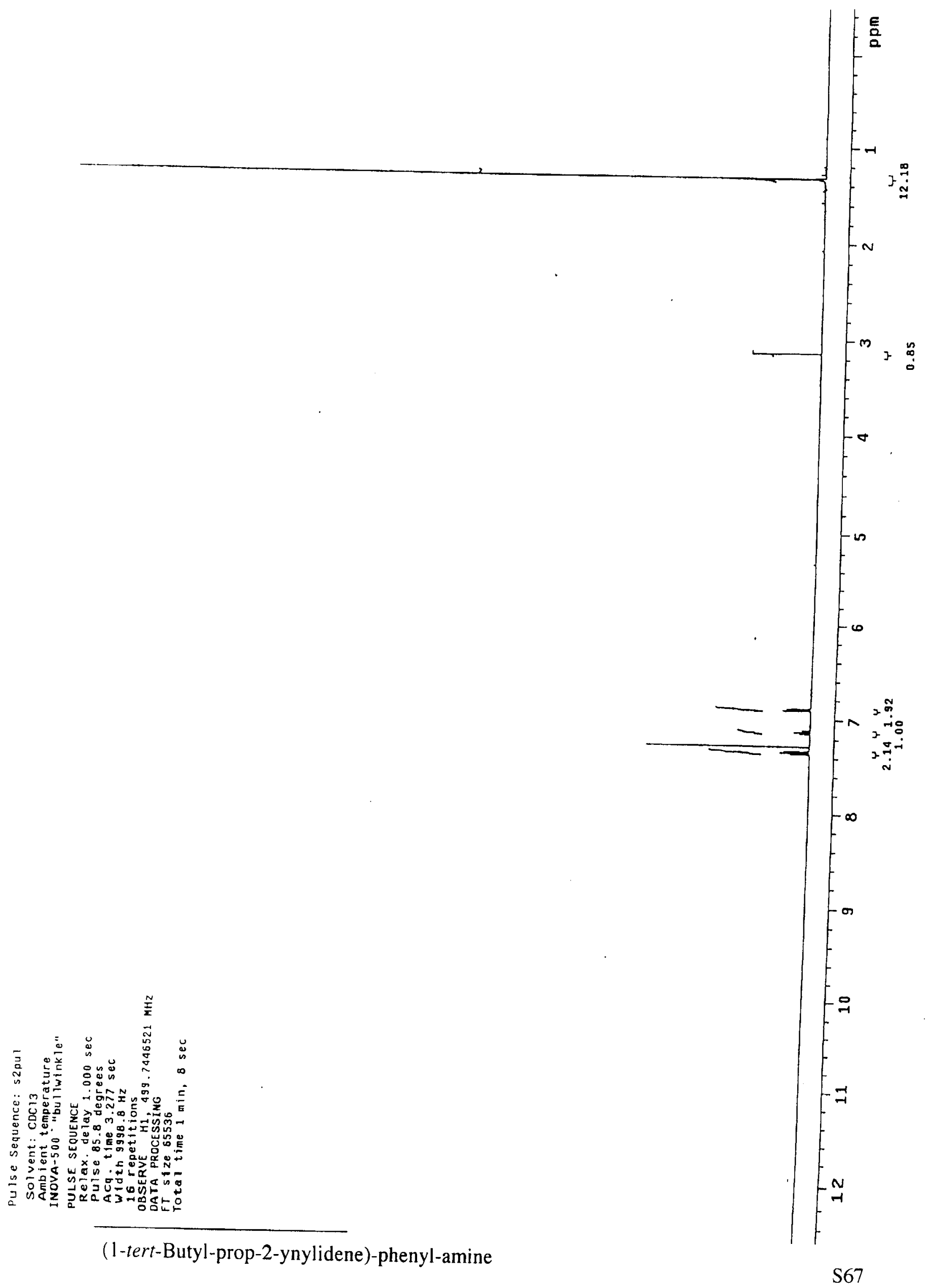




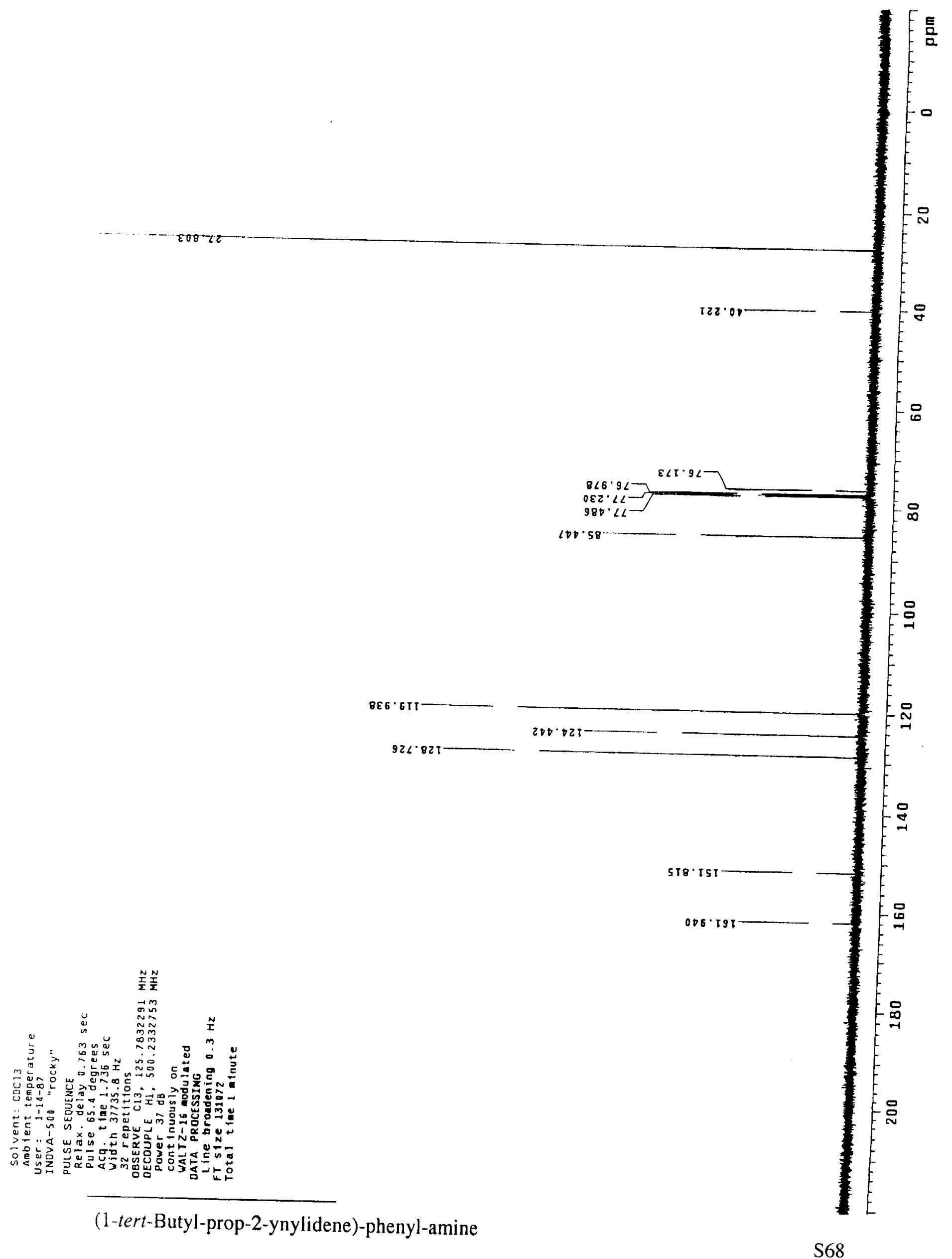




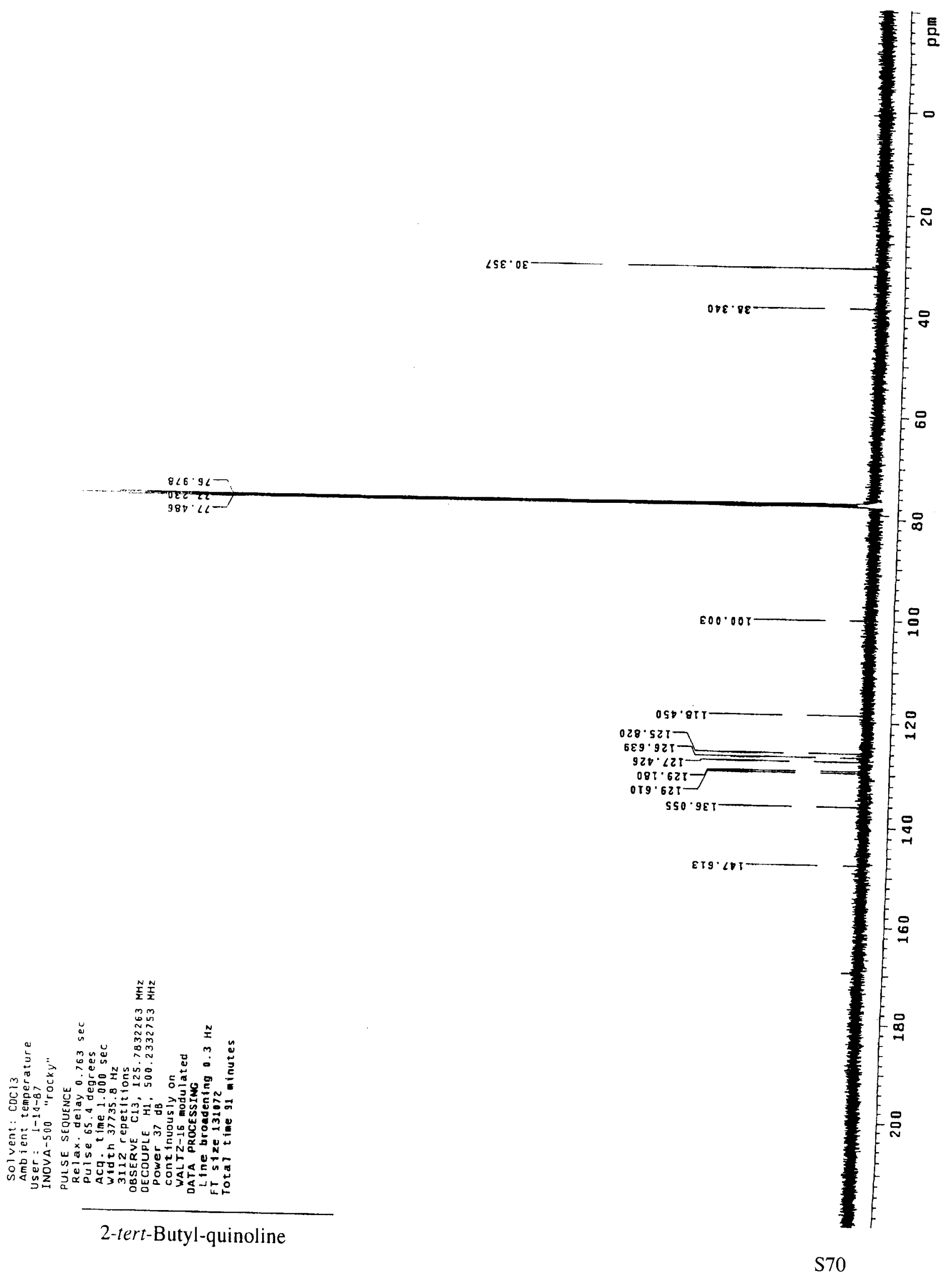




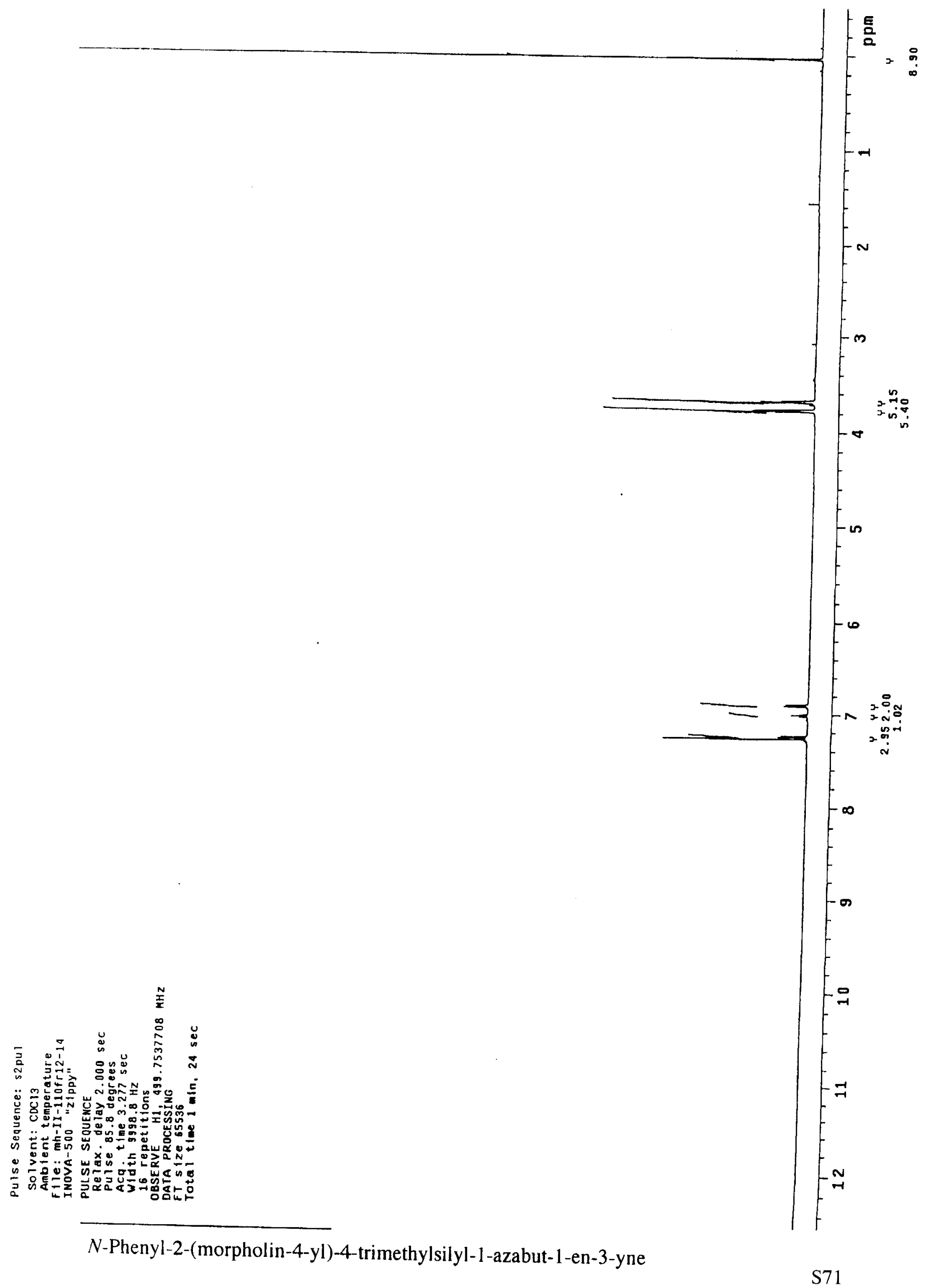




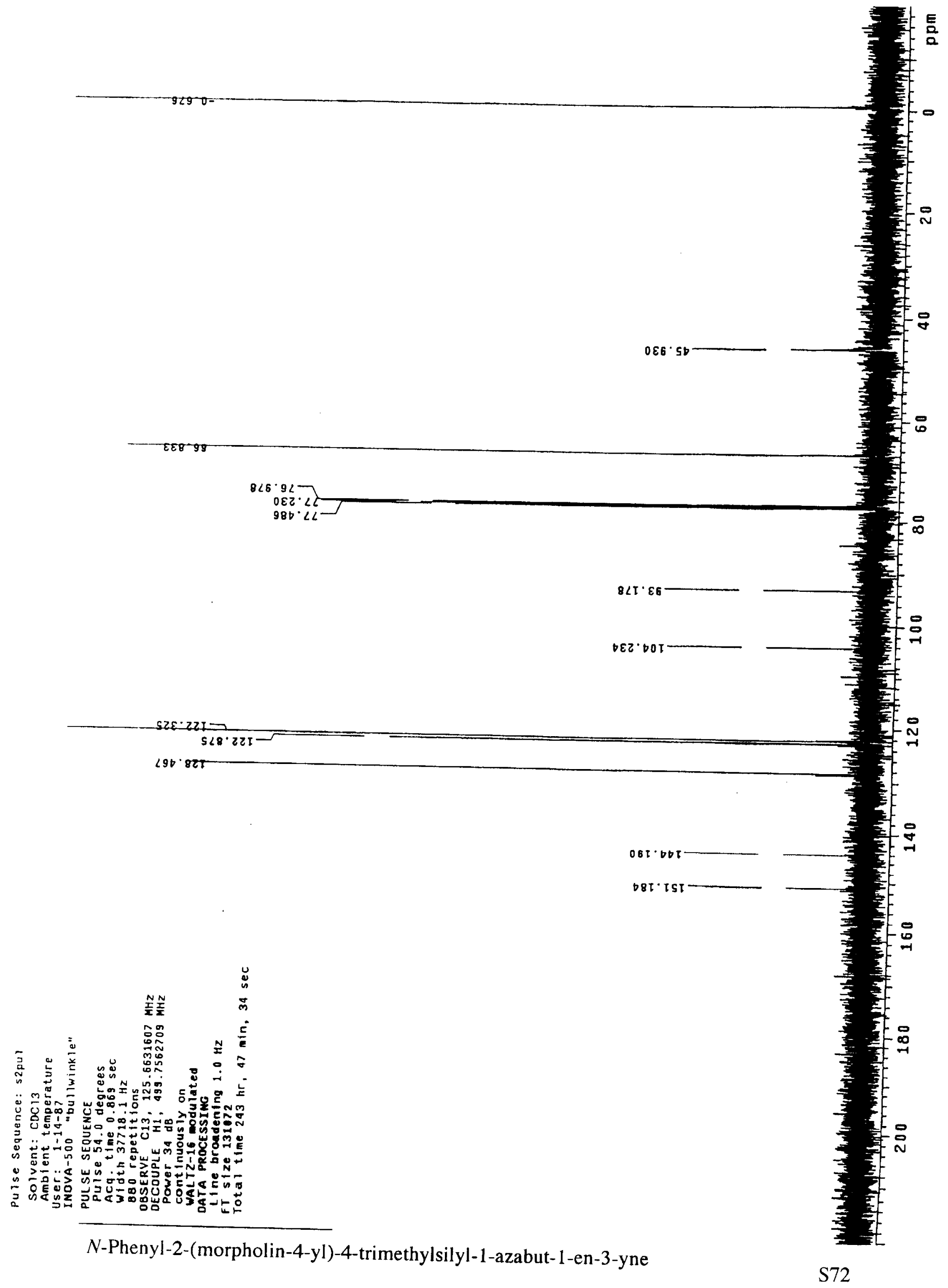




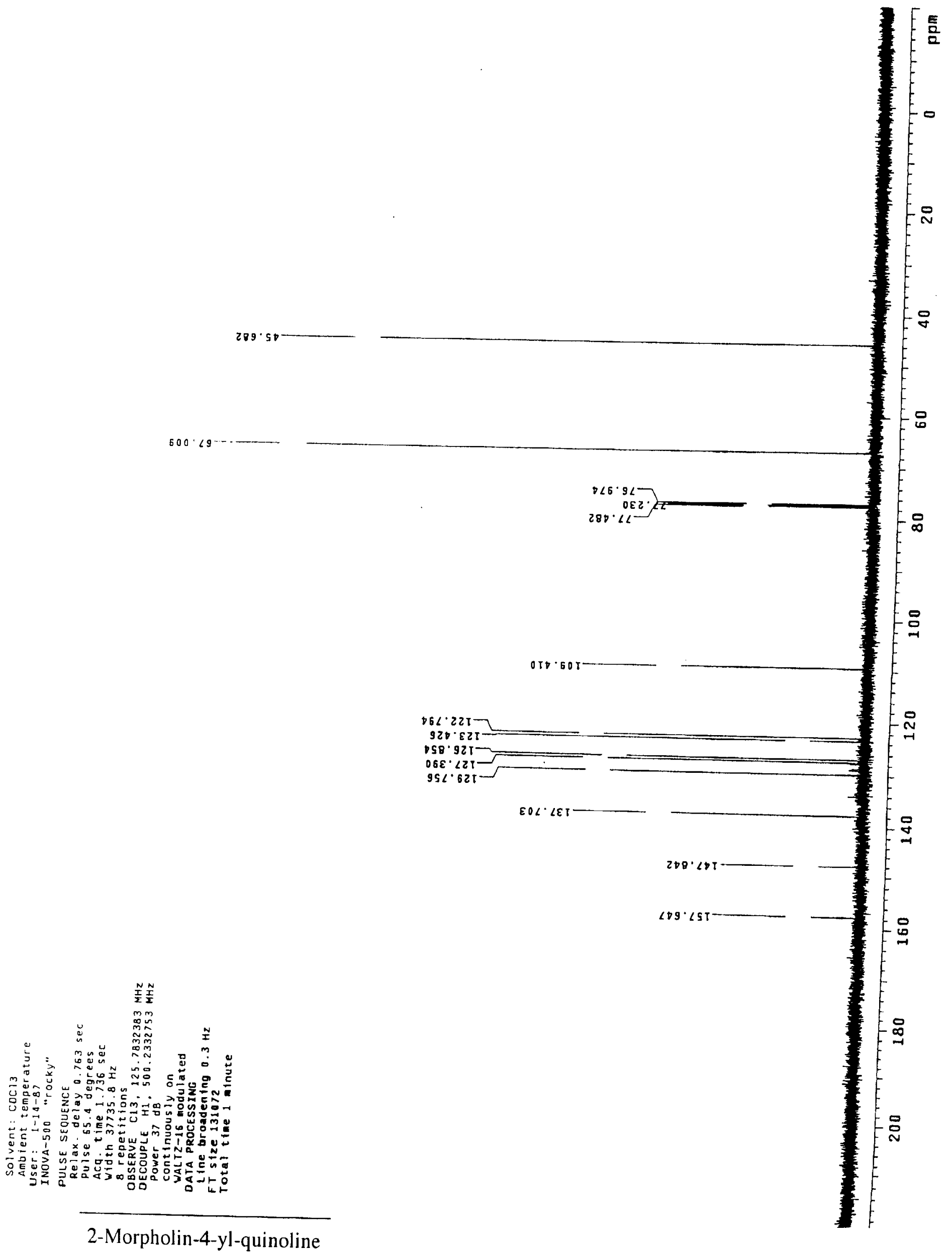




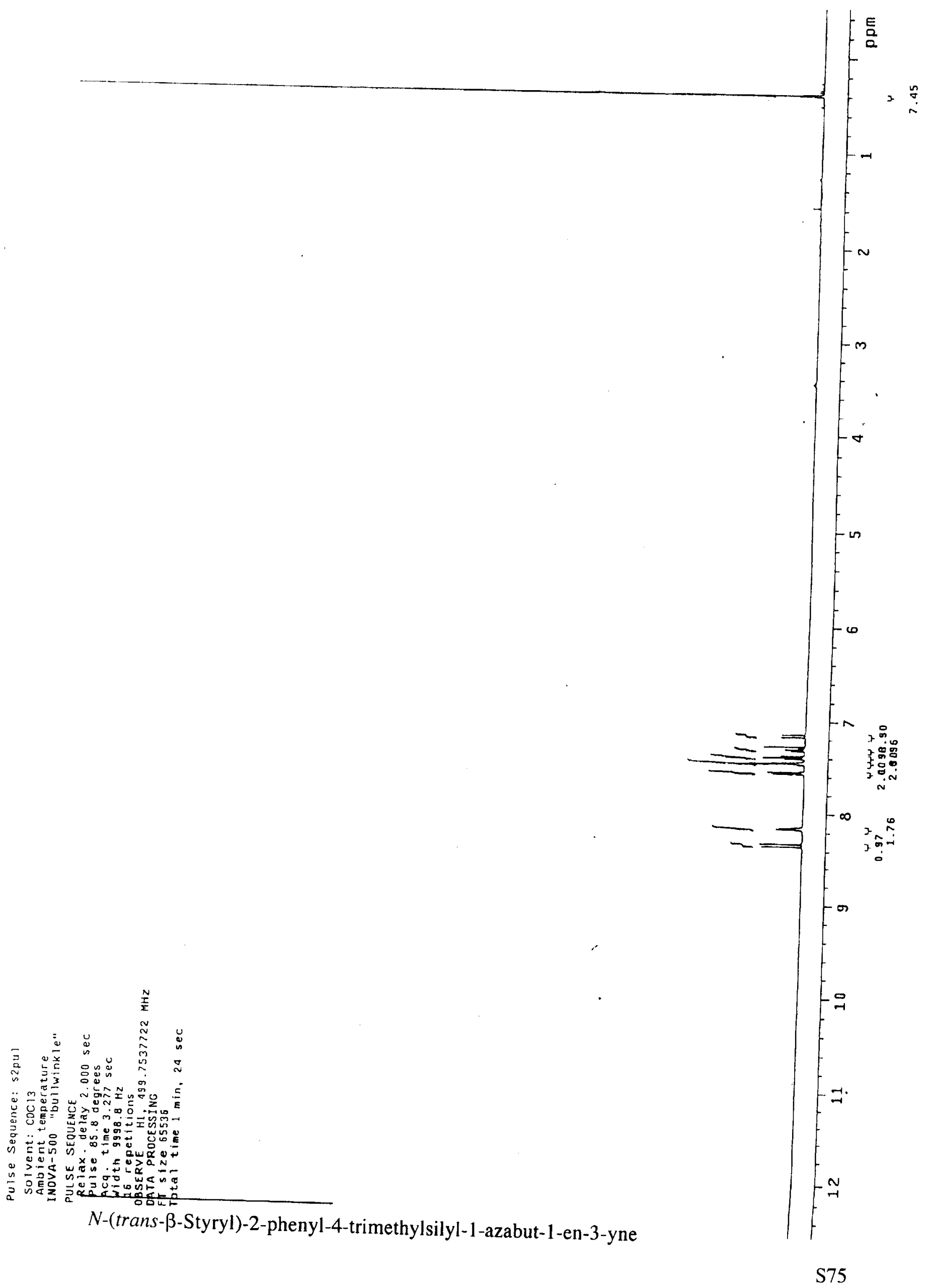




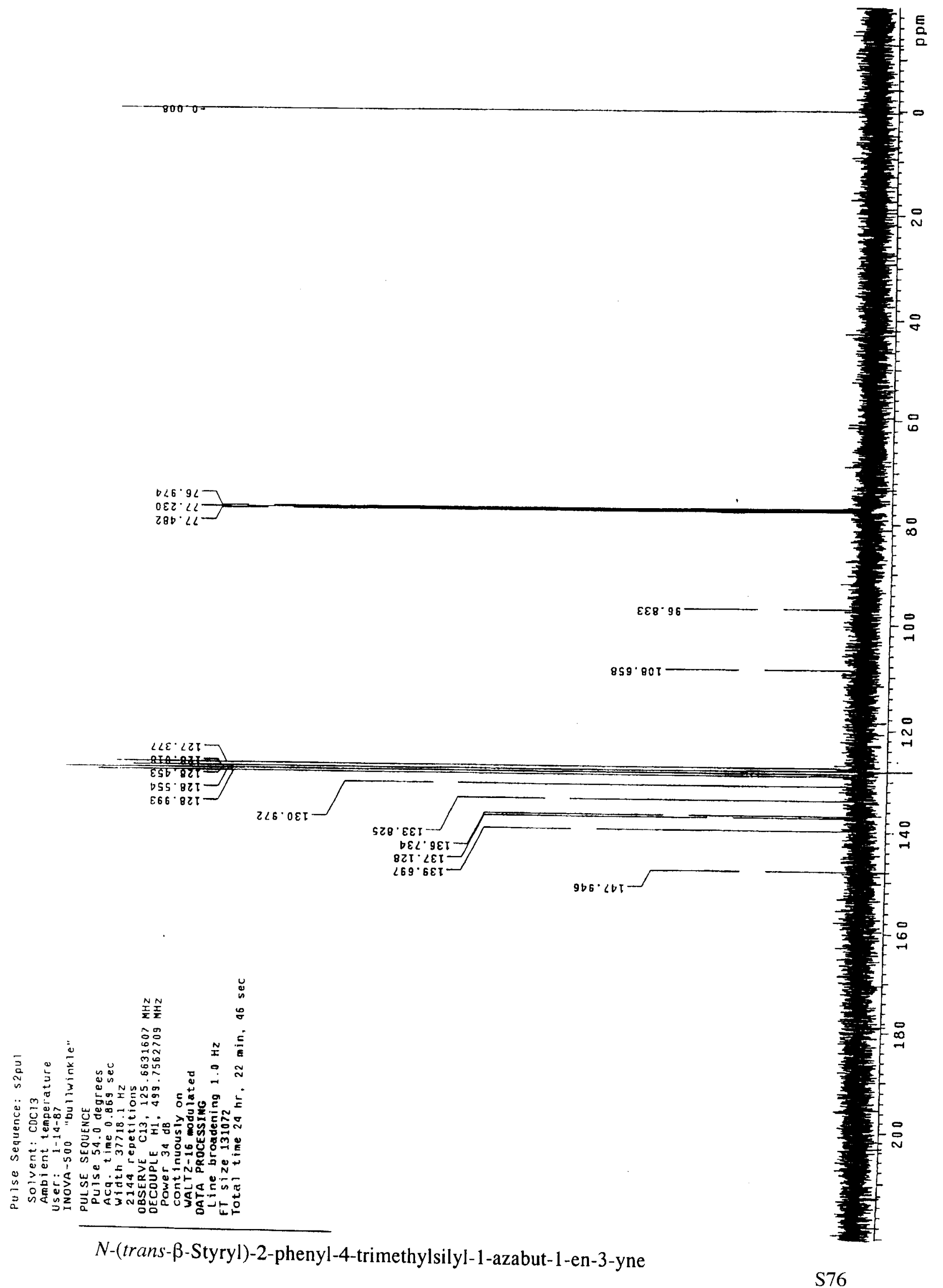



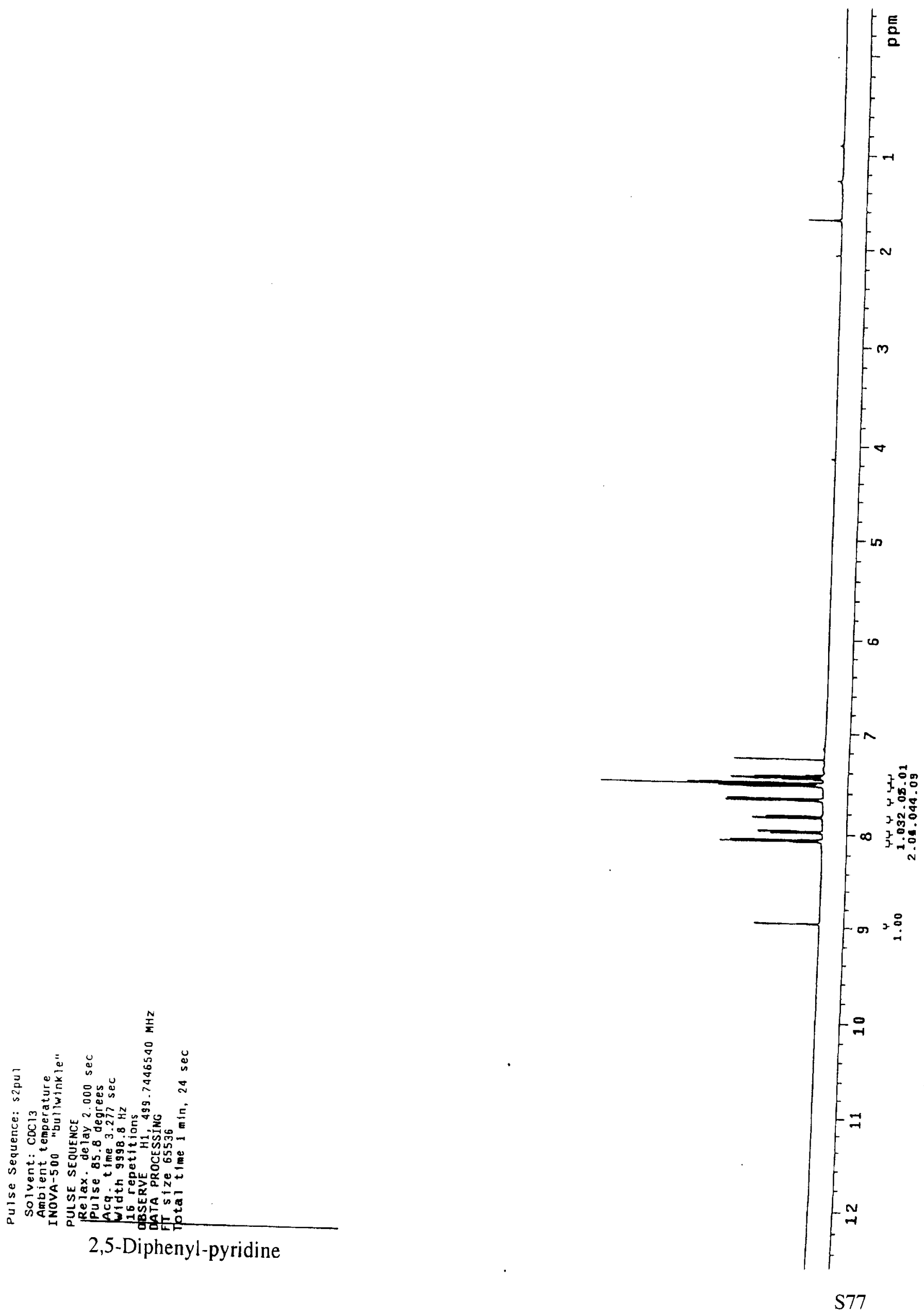


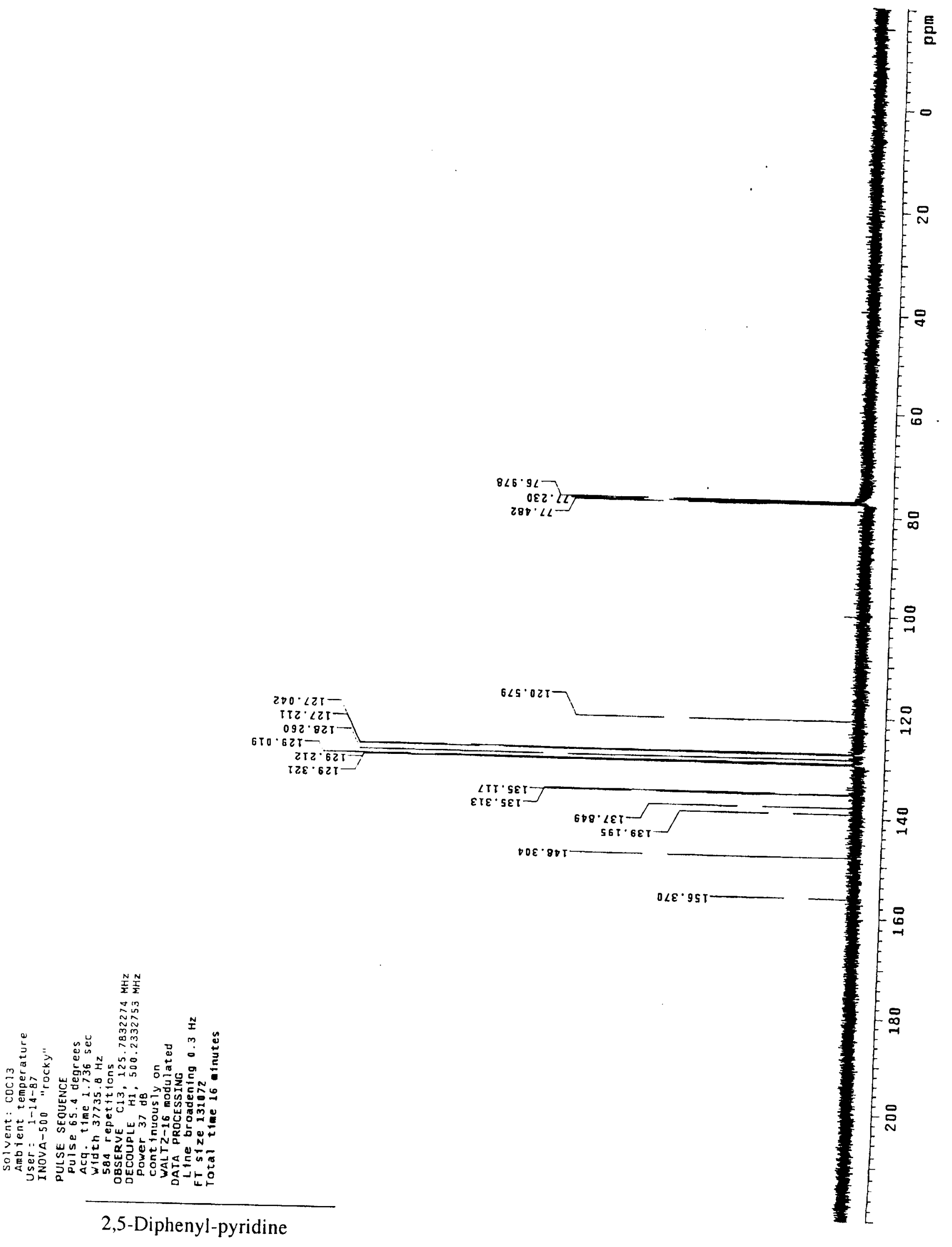




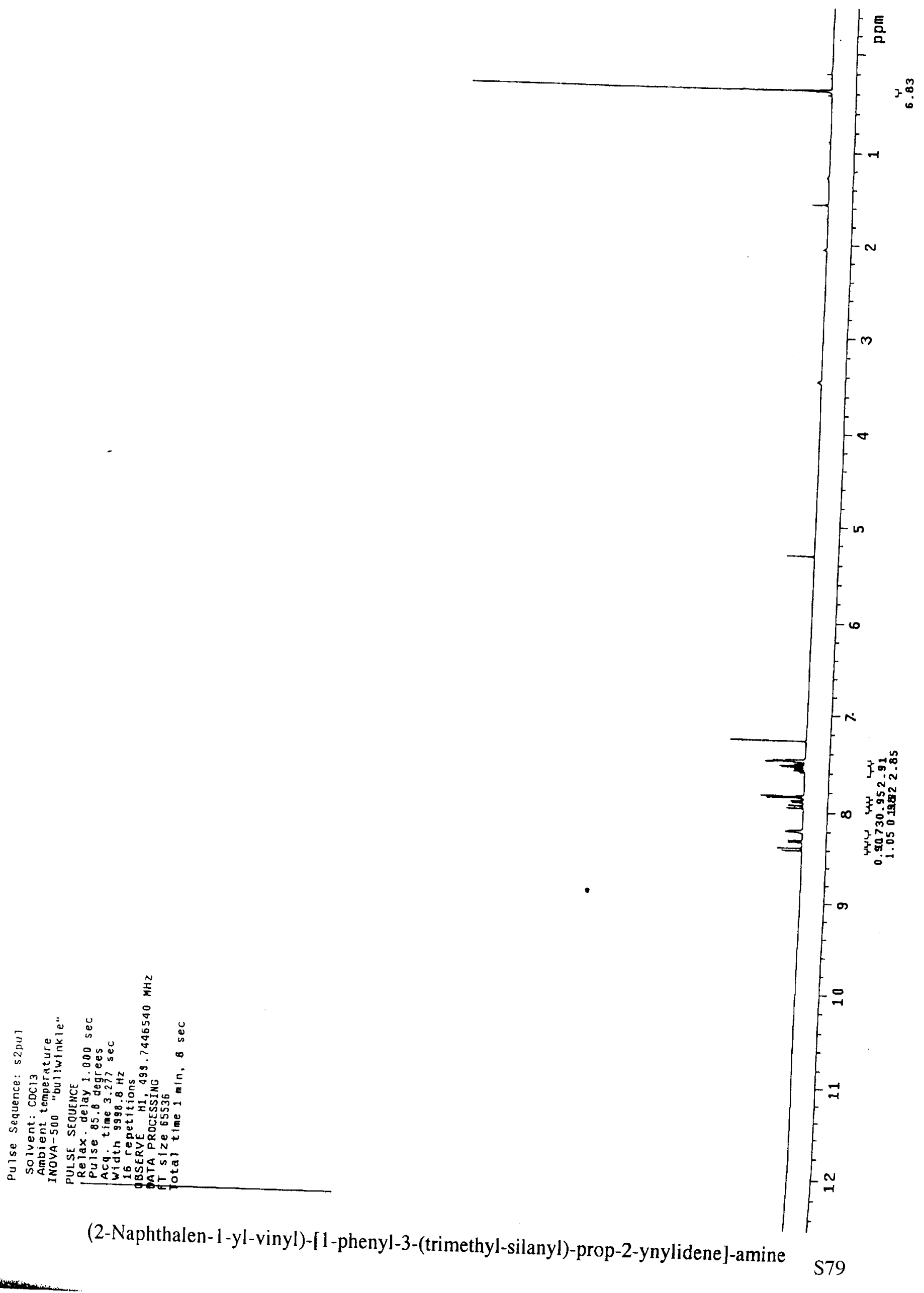




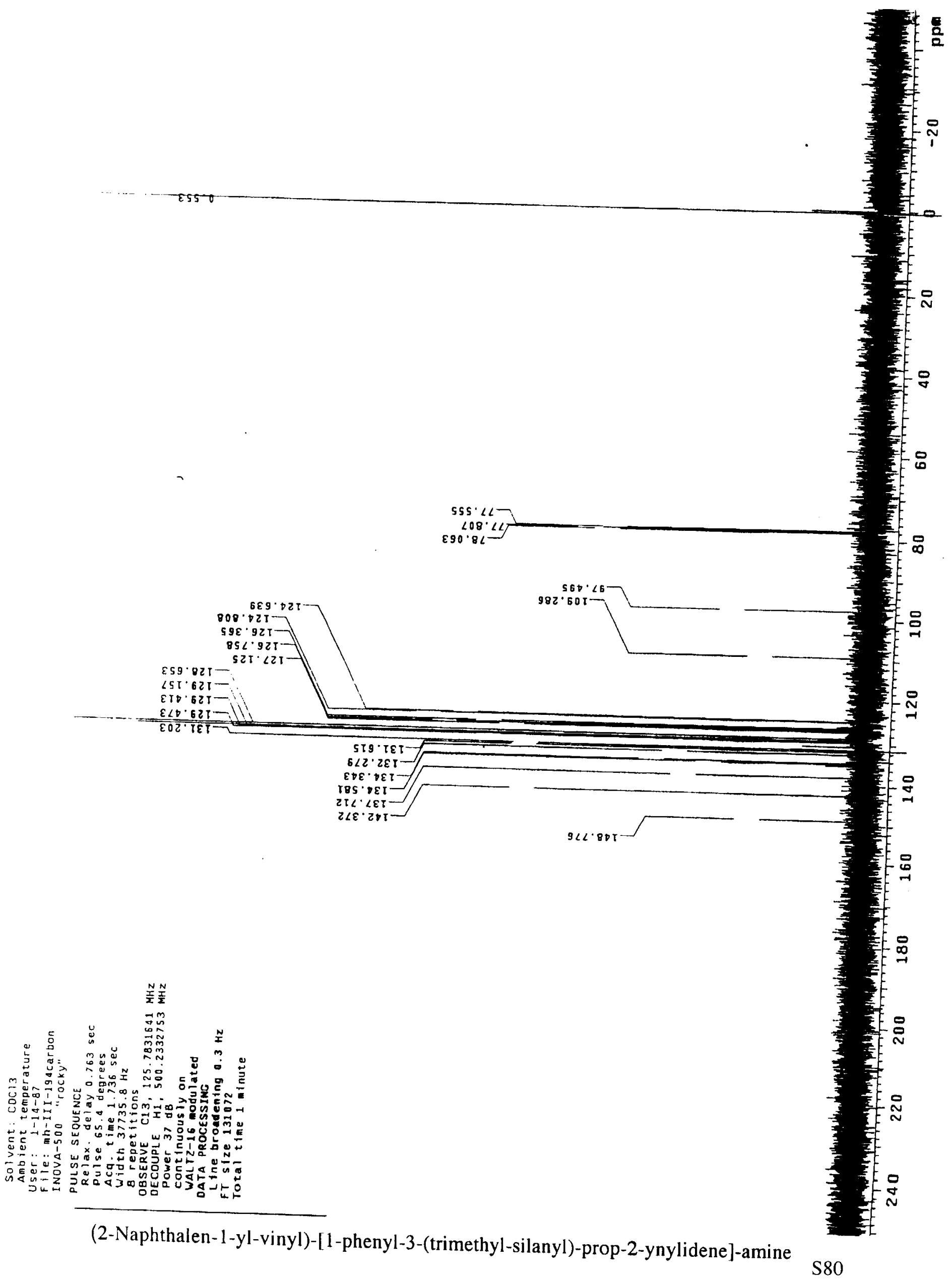




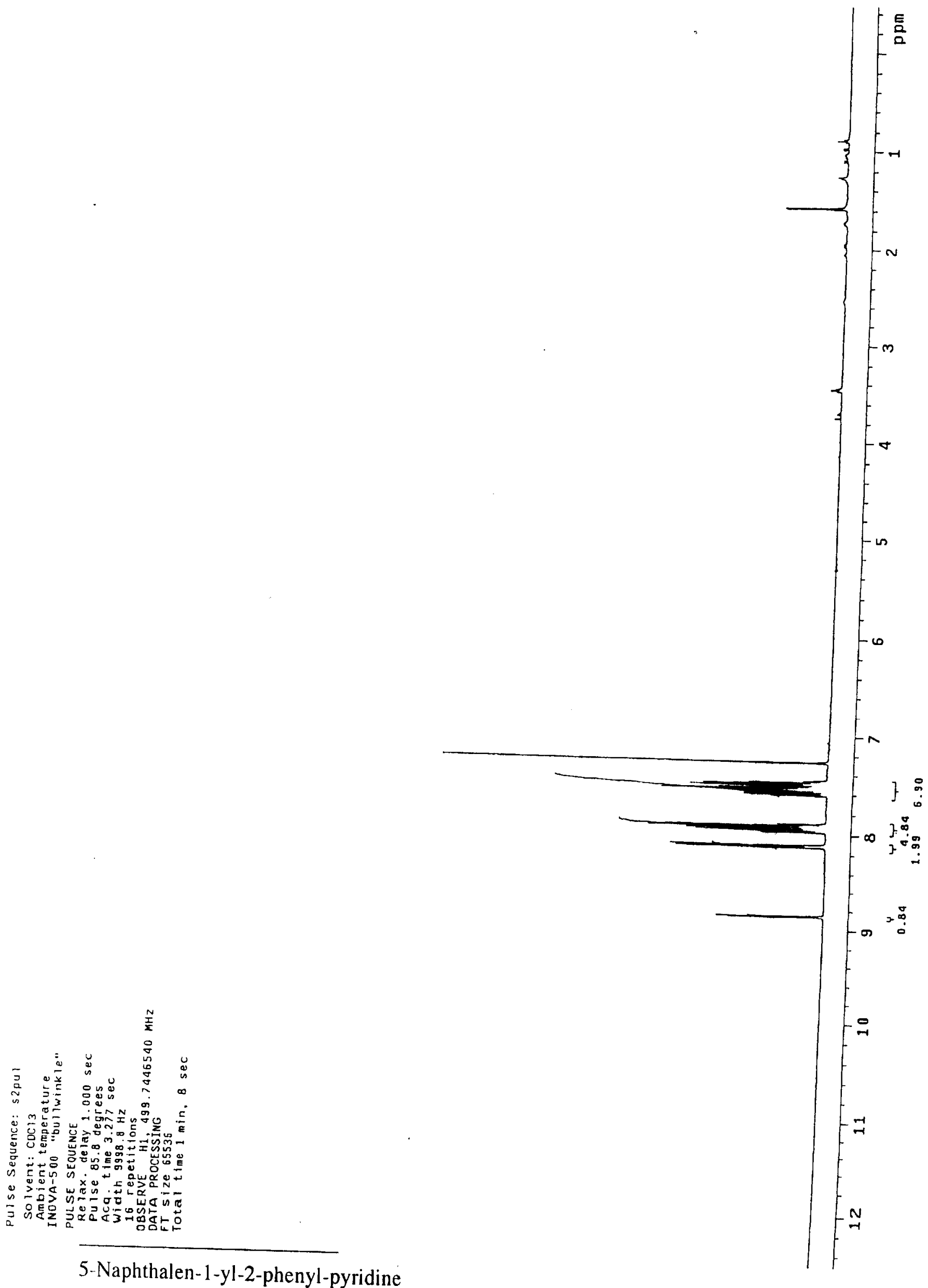




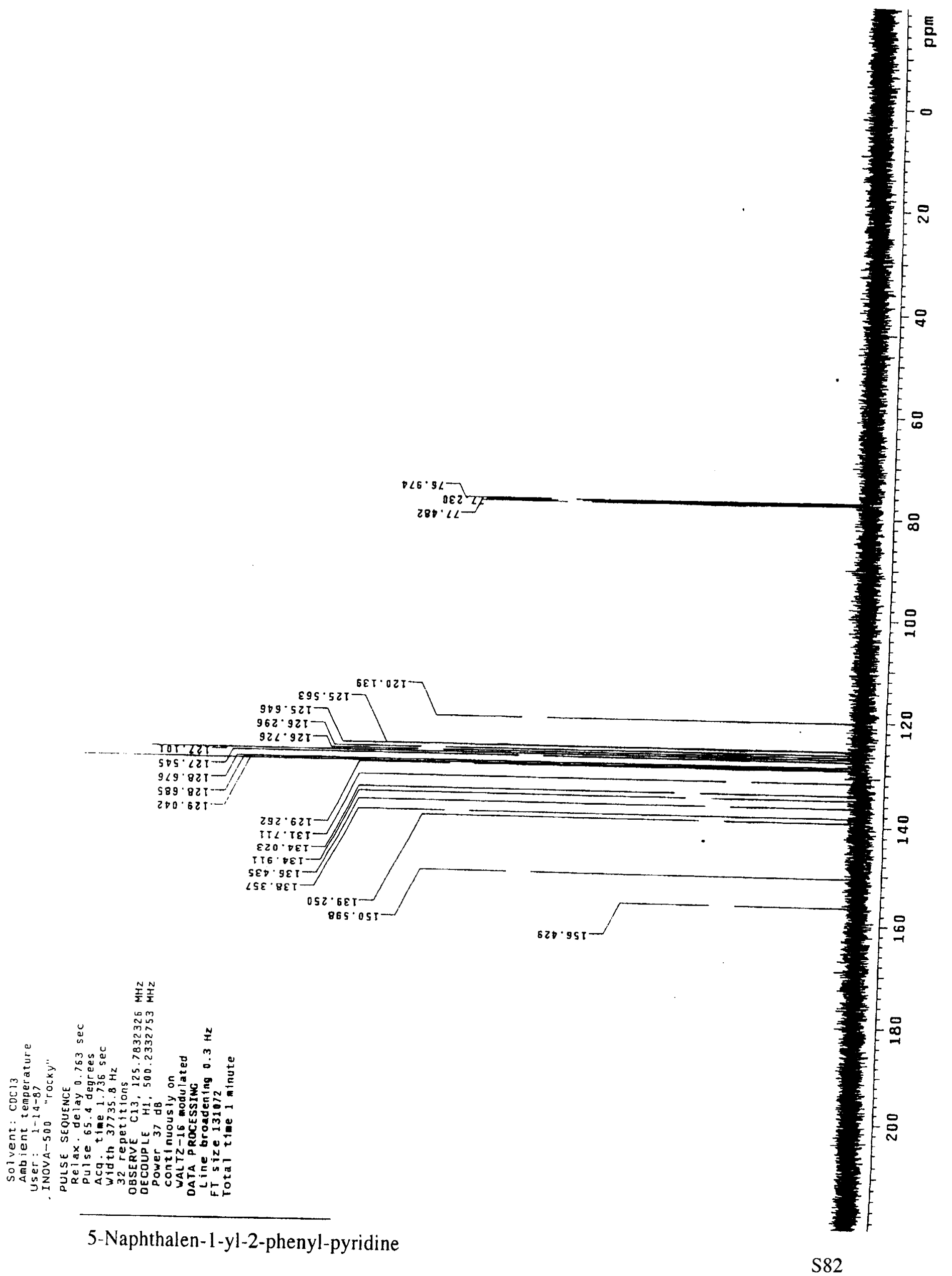




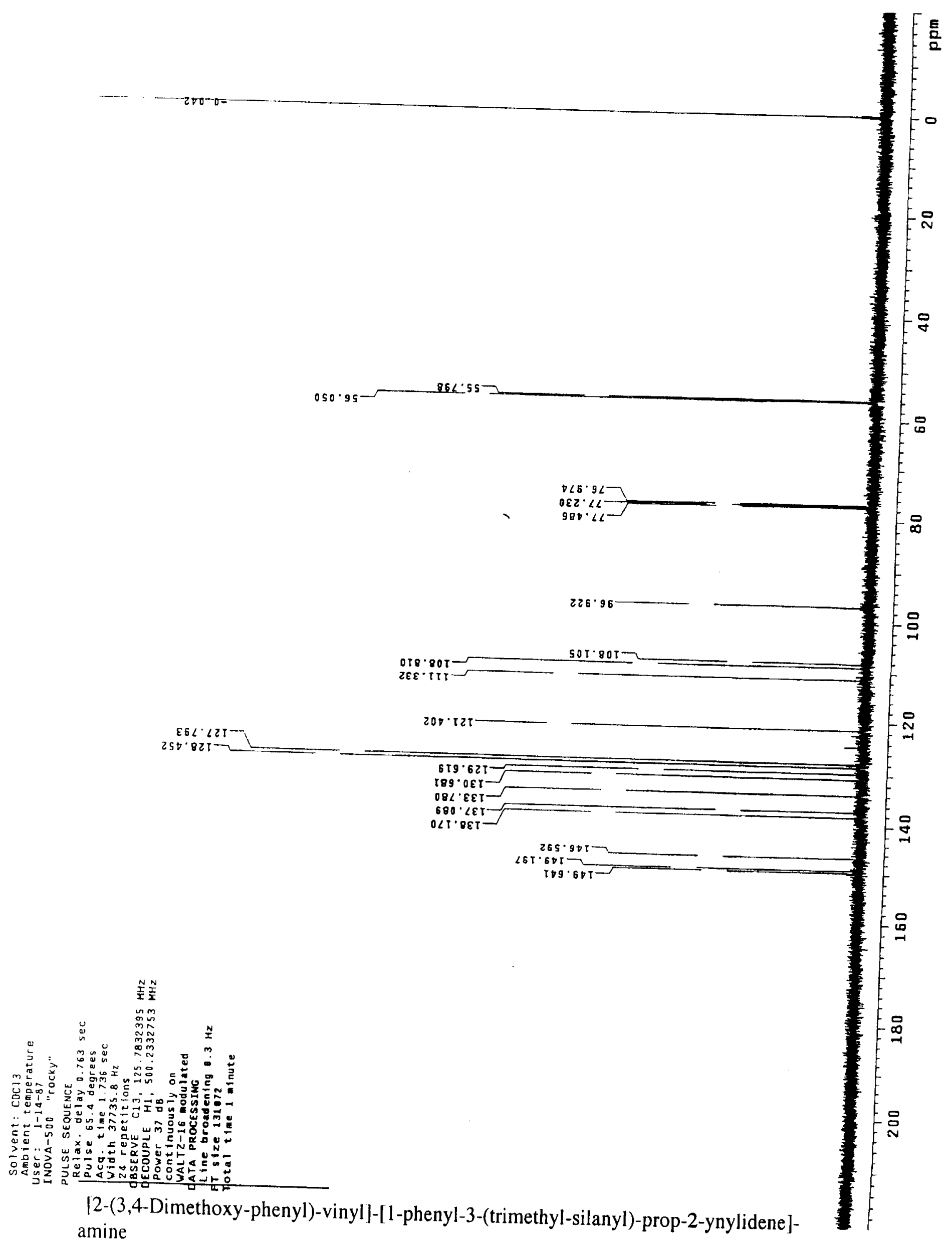




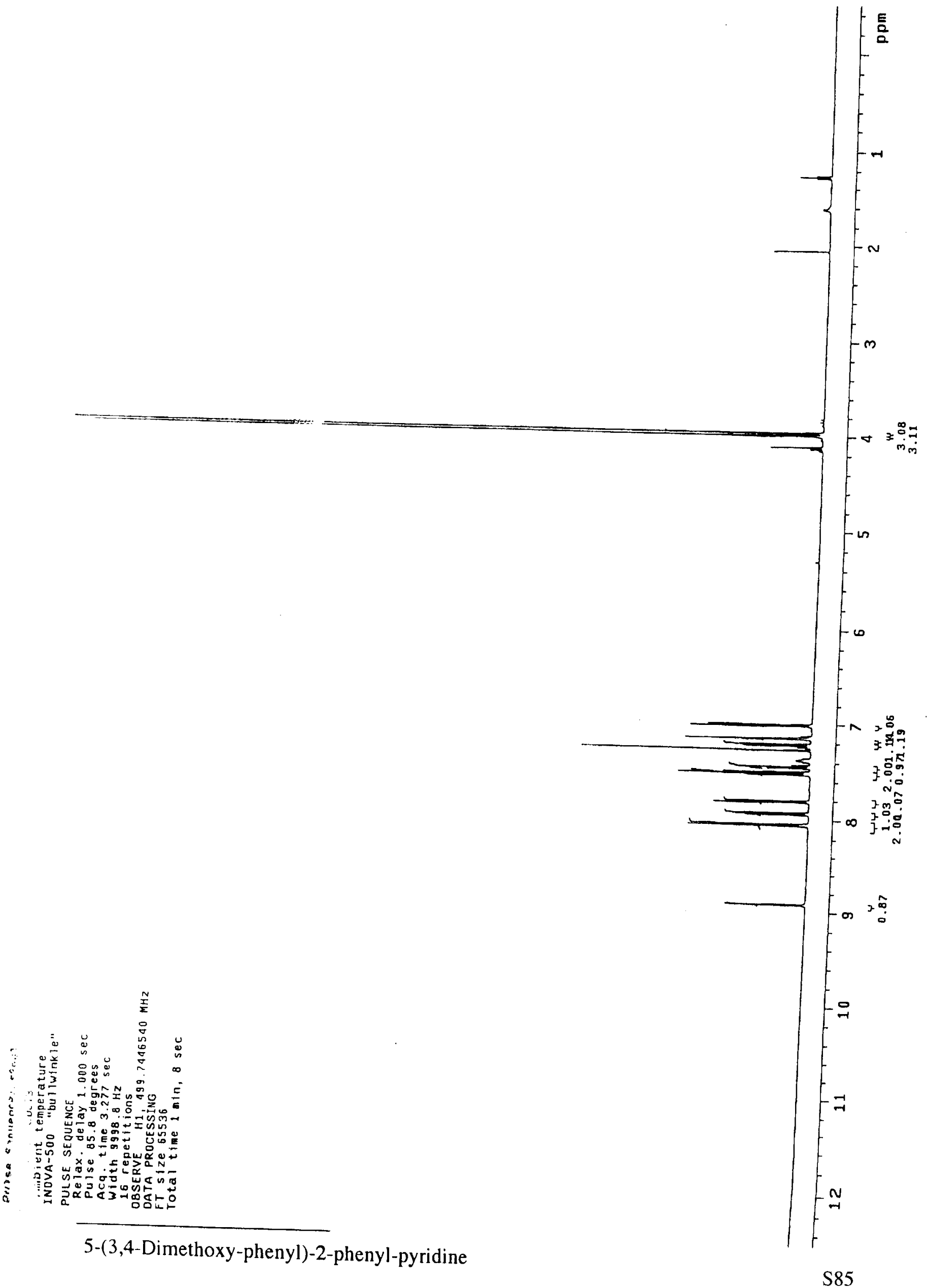




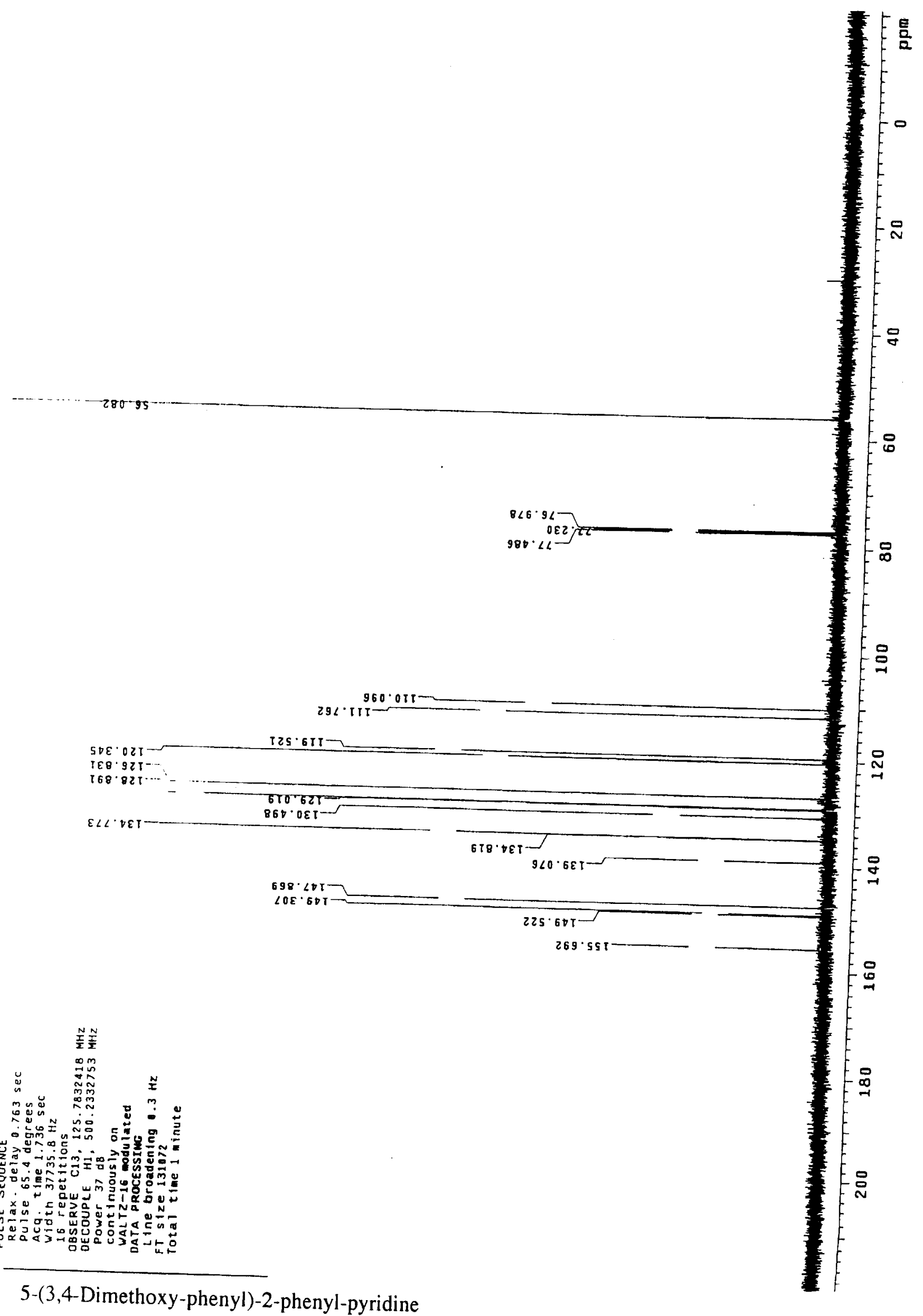




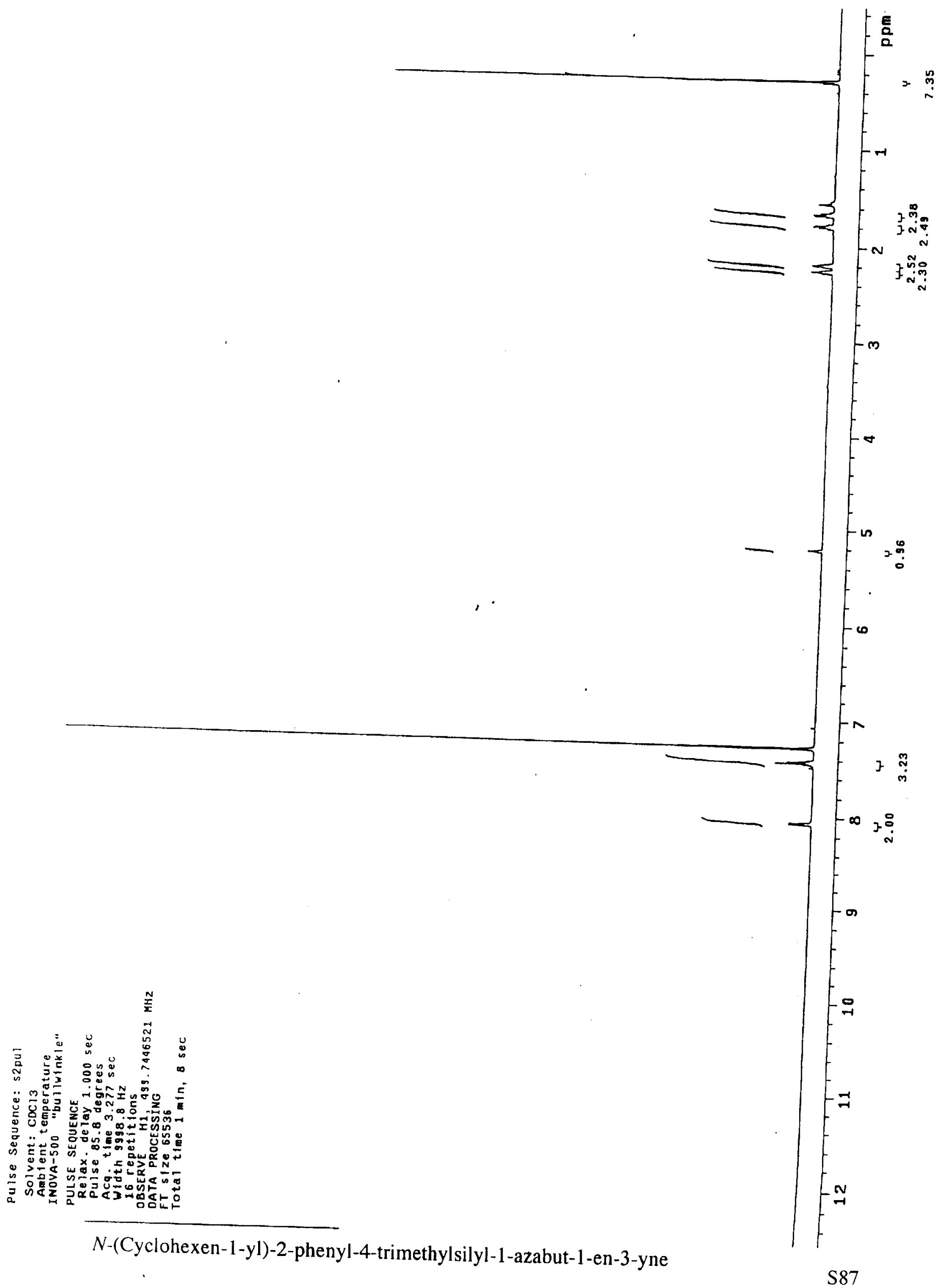




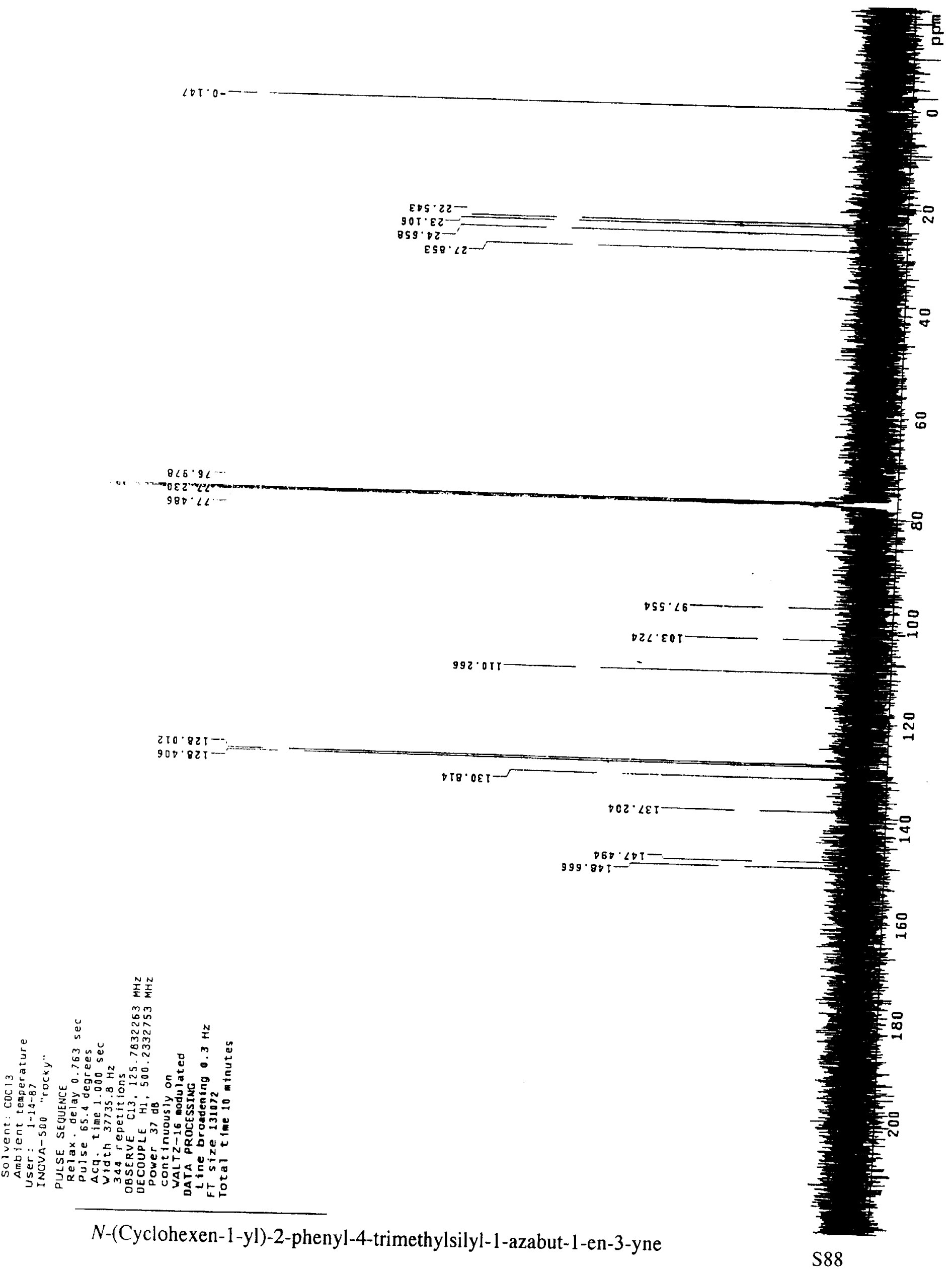




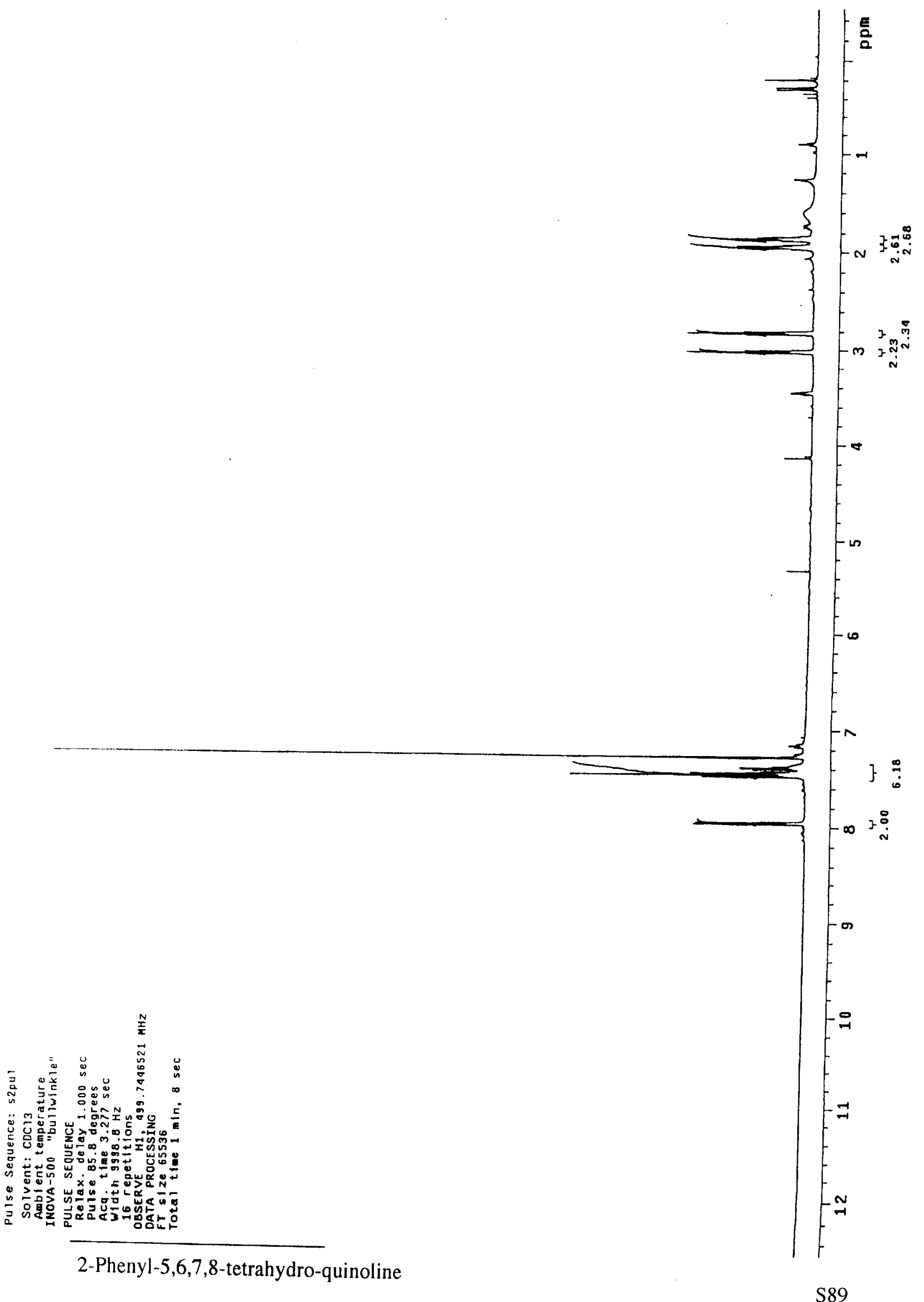




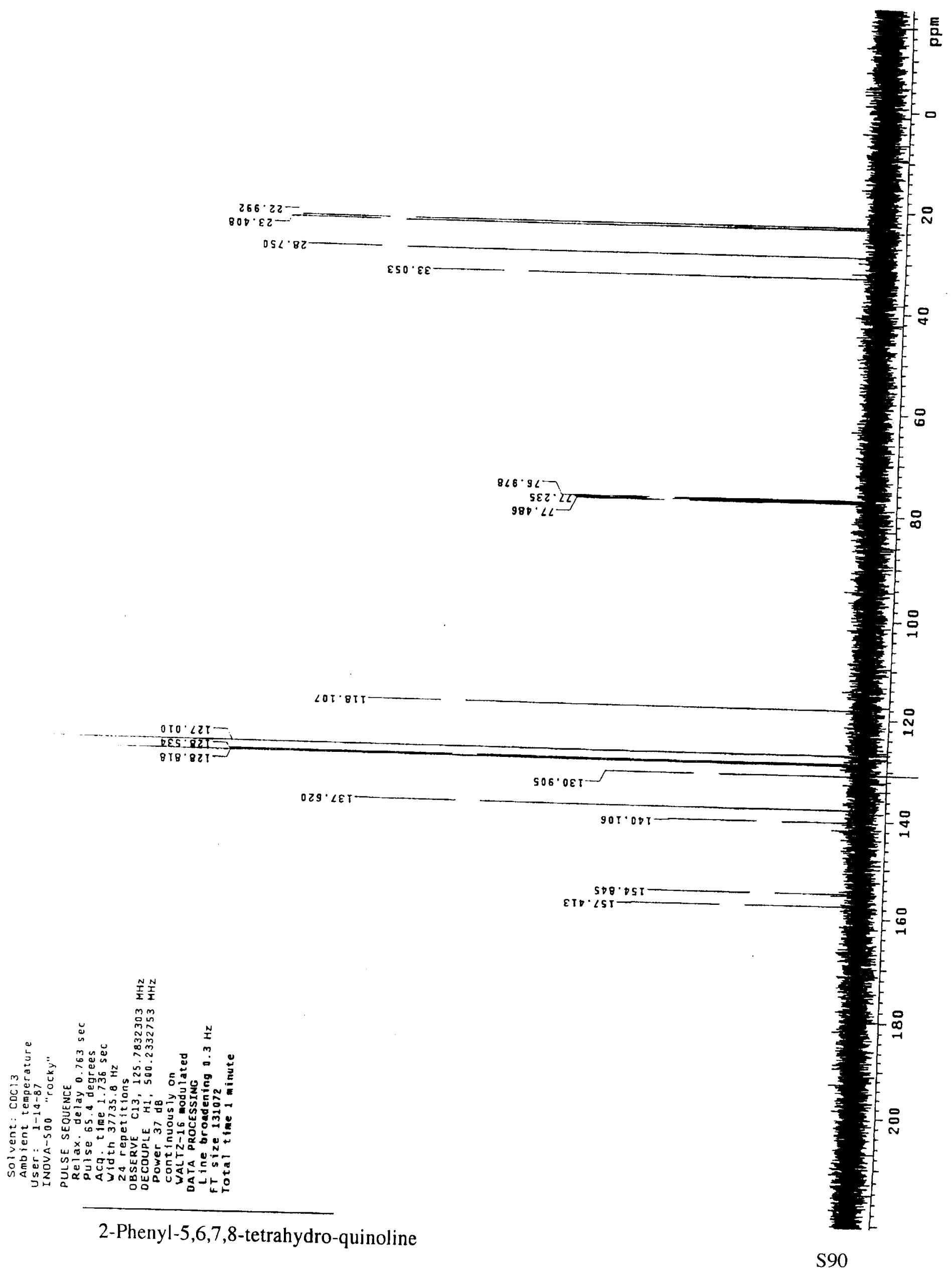




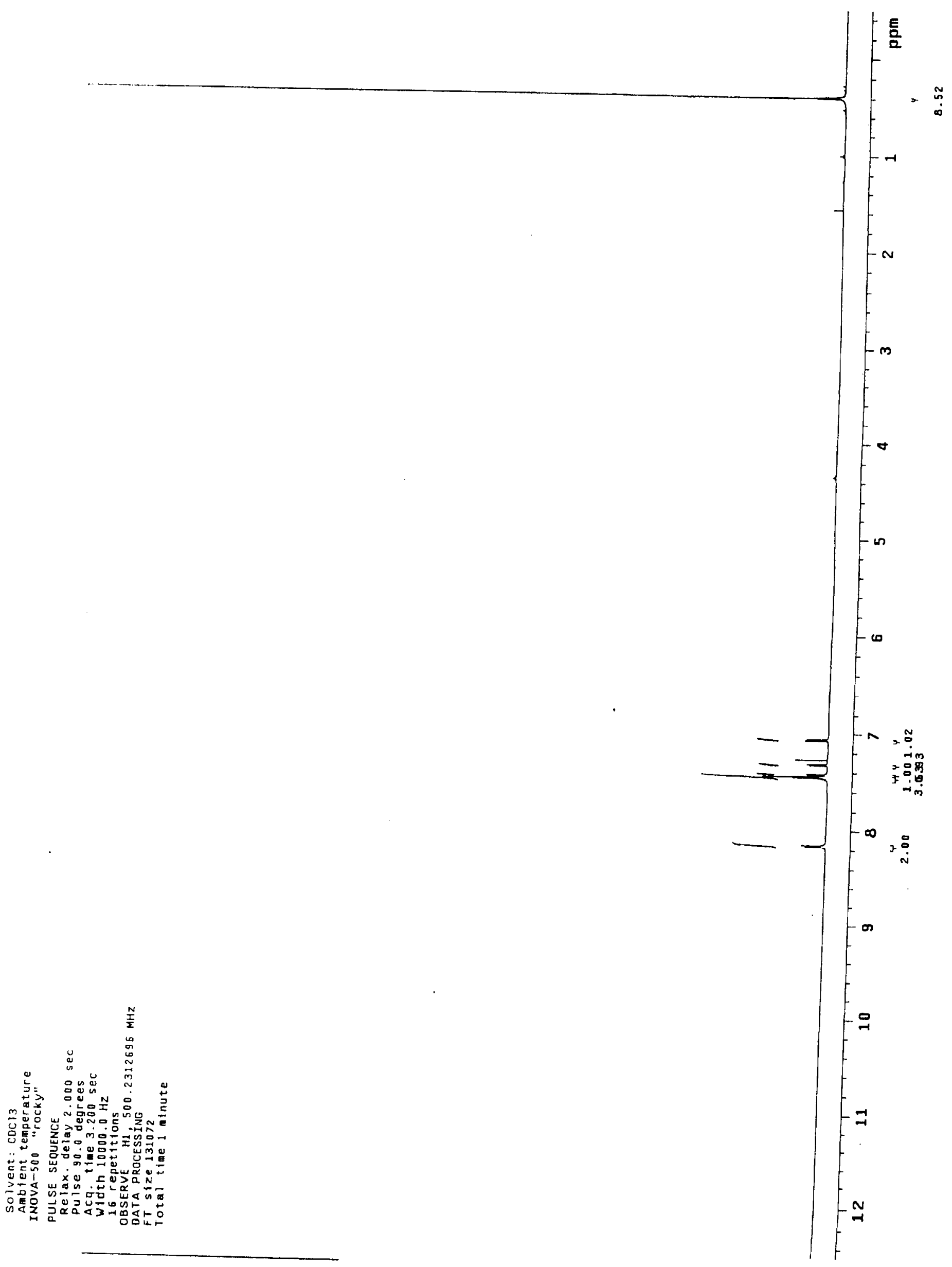

$N$-(Thiophen-2-yl)-2-(phenyl)-4-trimethylsilyl-1-azabut-1-en-3-yne 


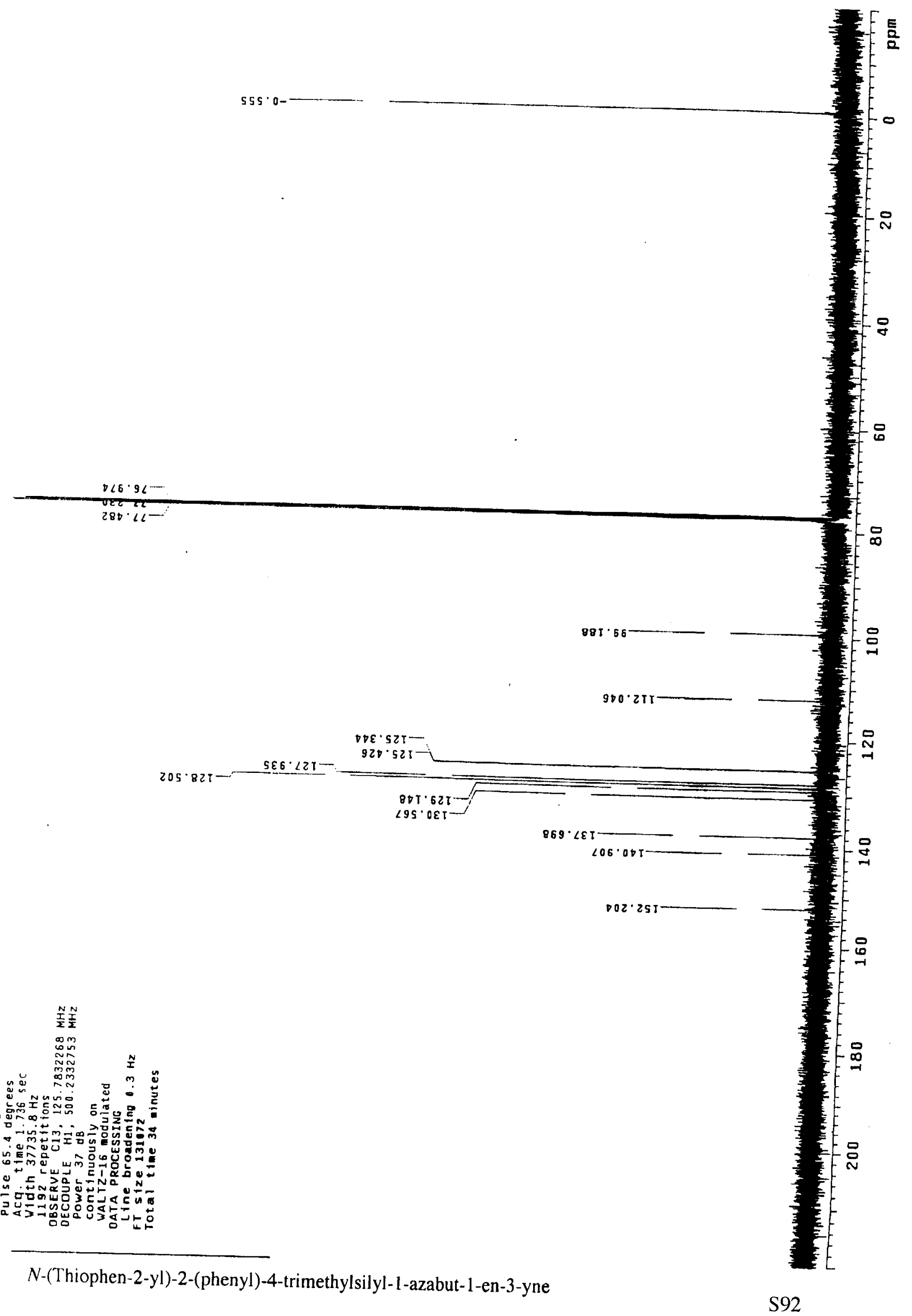




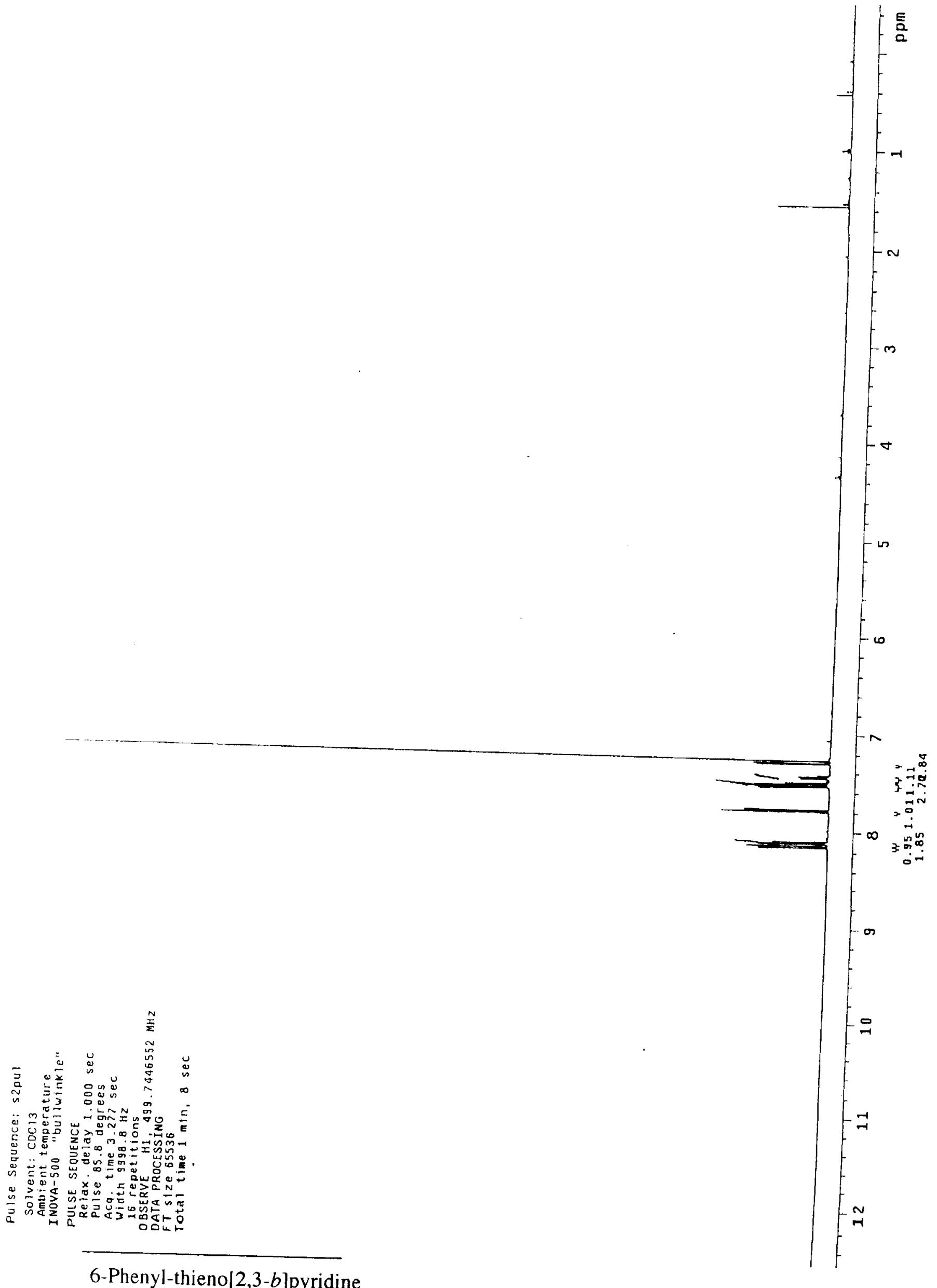

6-Phenyl-thieno[2,3-b]pyridine 


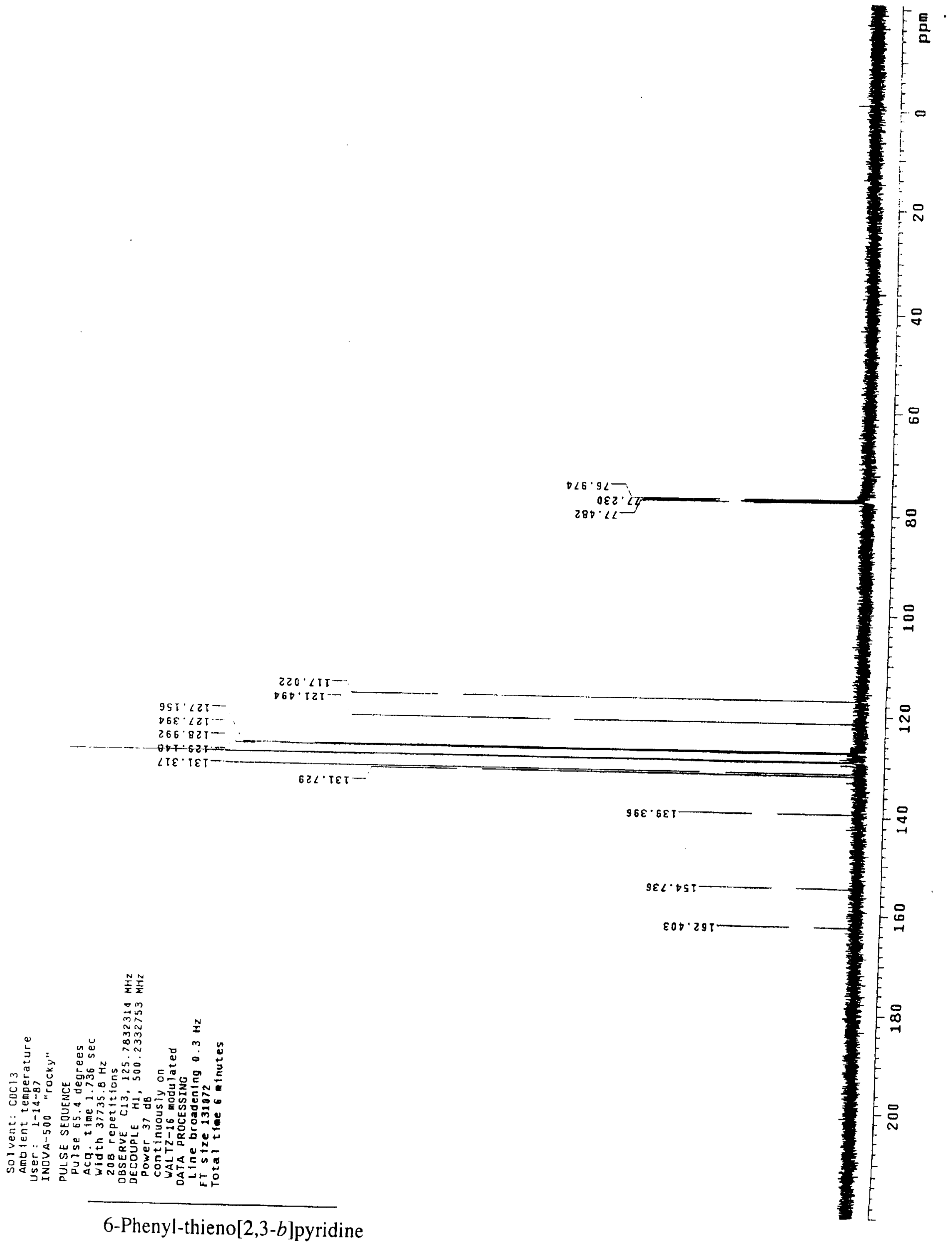




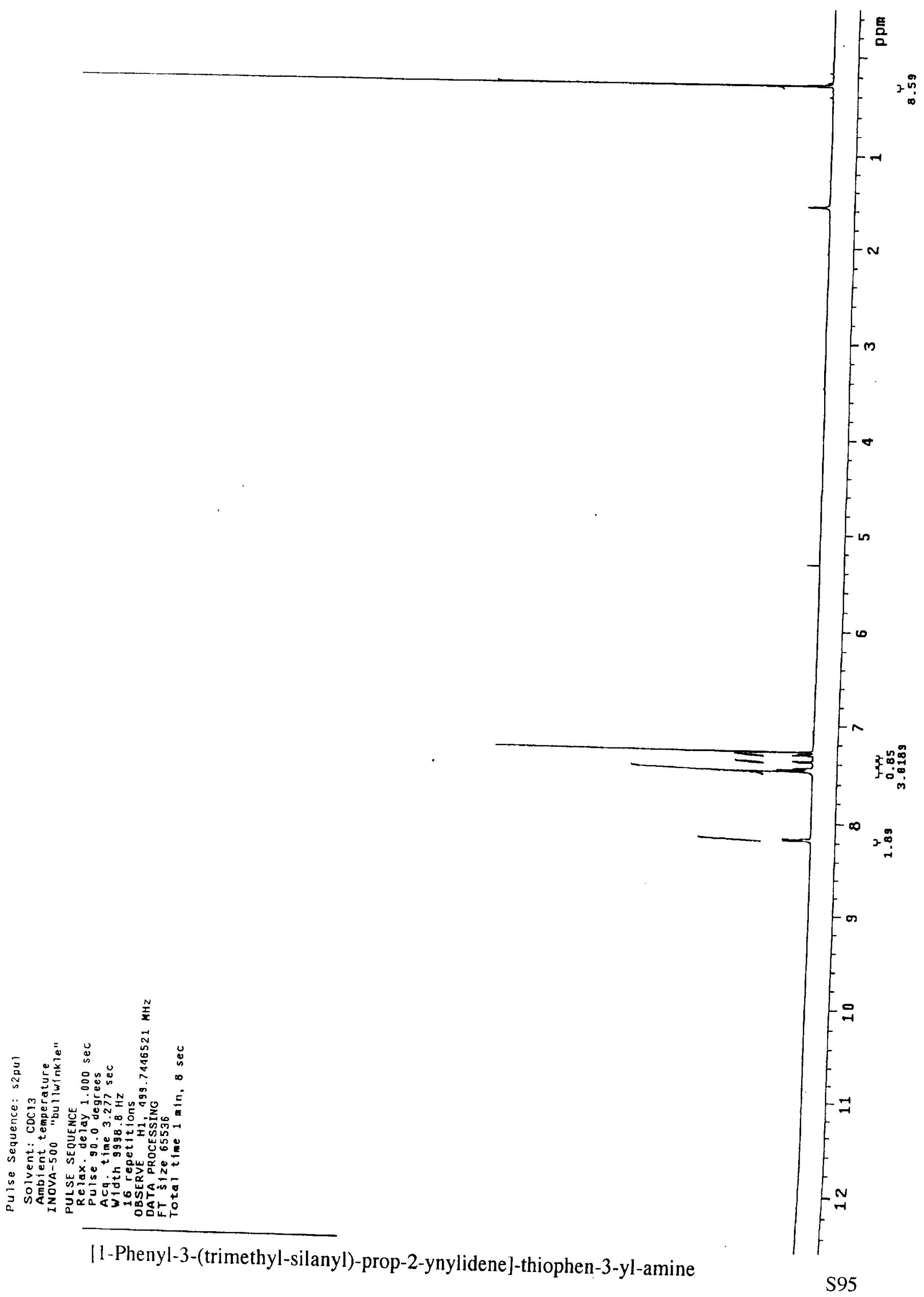




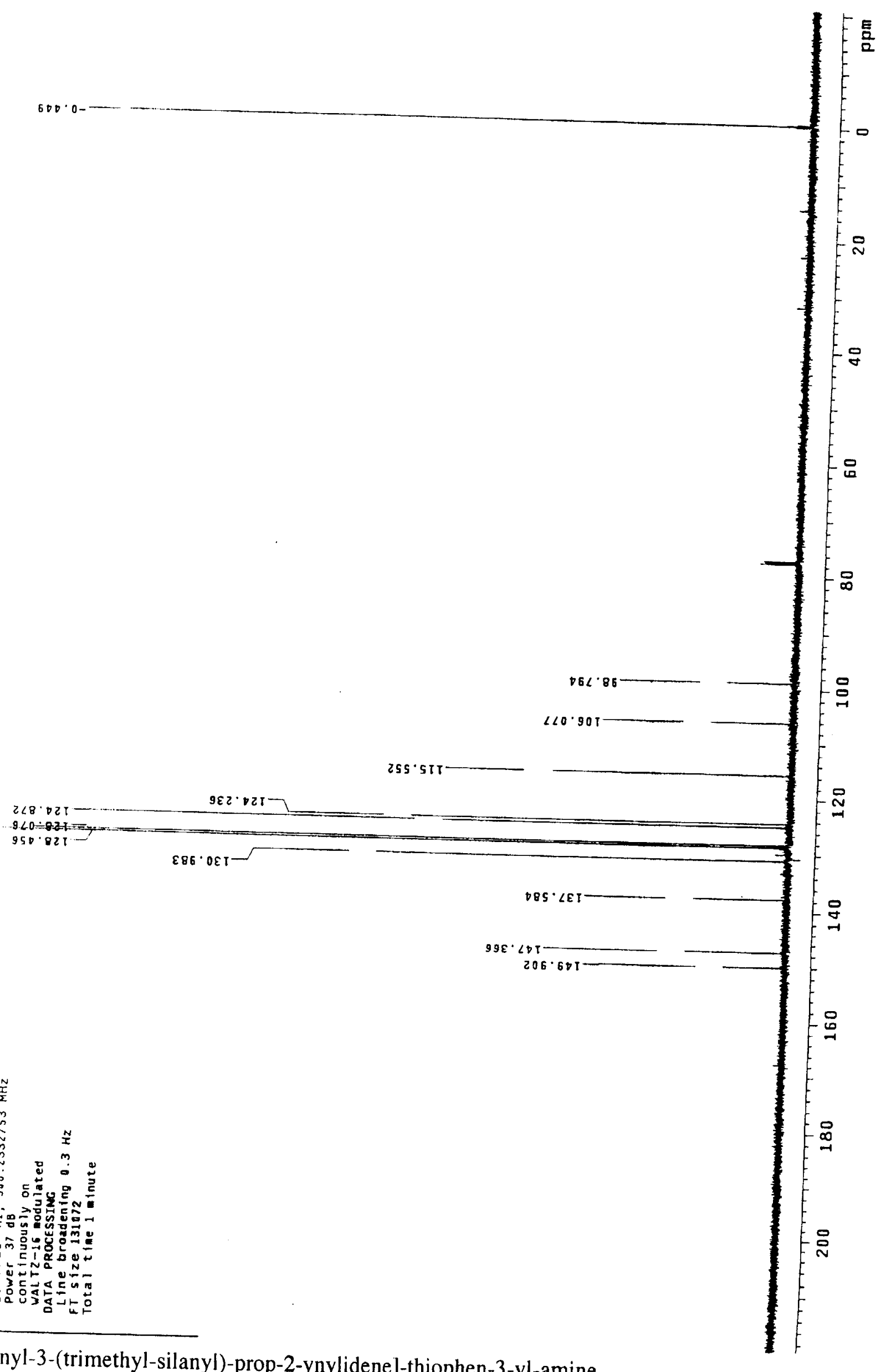

[1-Phenyl-3-(trimethyl-silanyl)-prop-2-ynylidene]-thiophen-3-yl-amine 


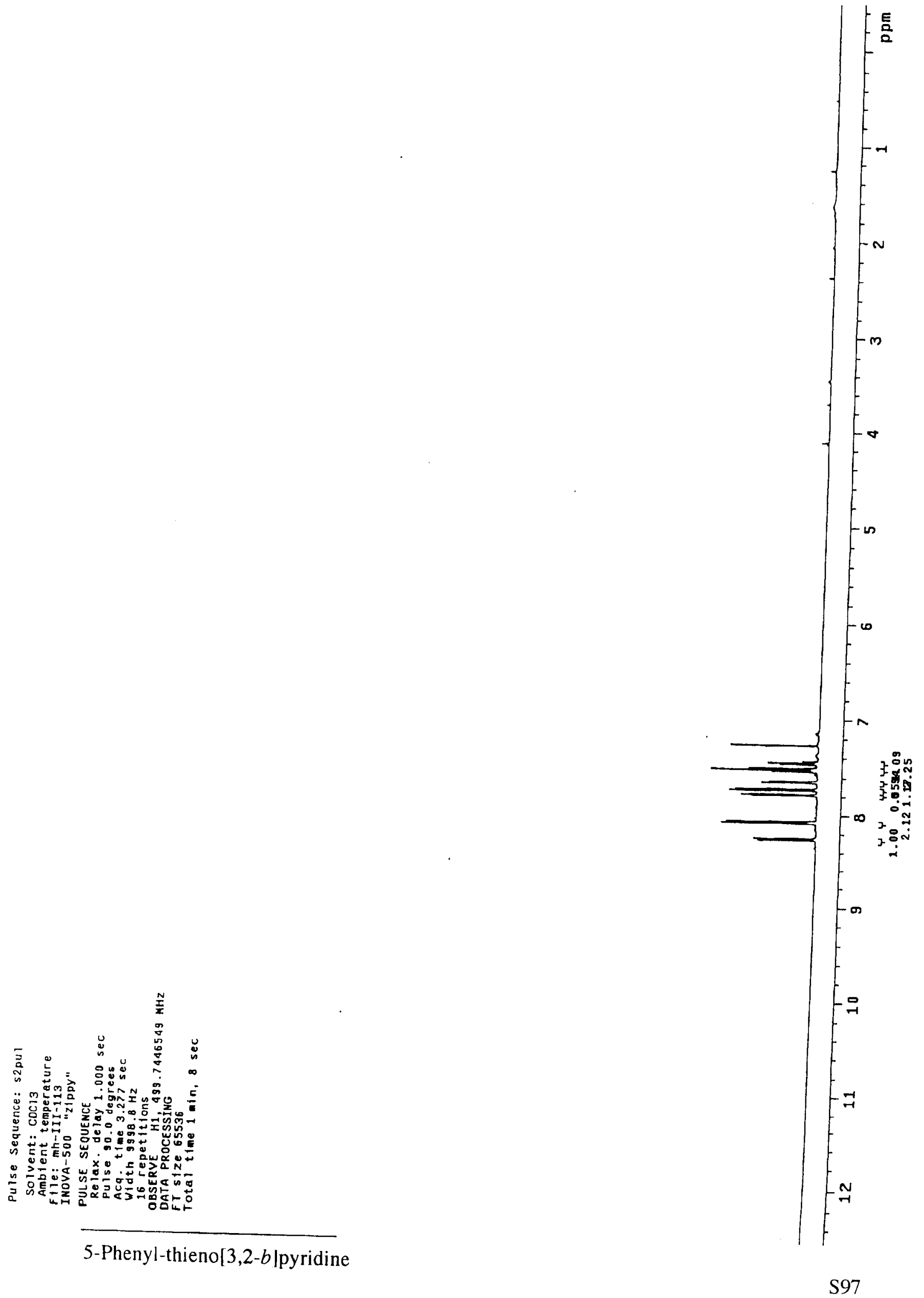




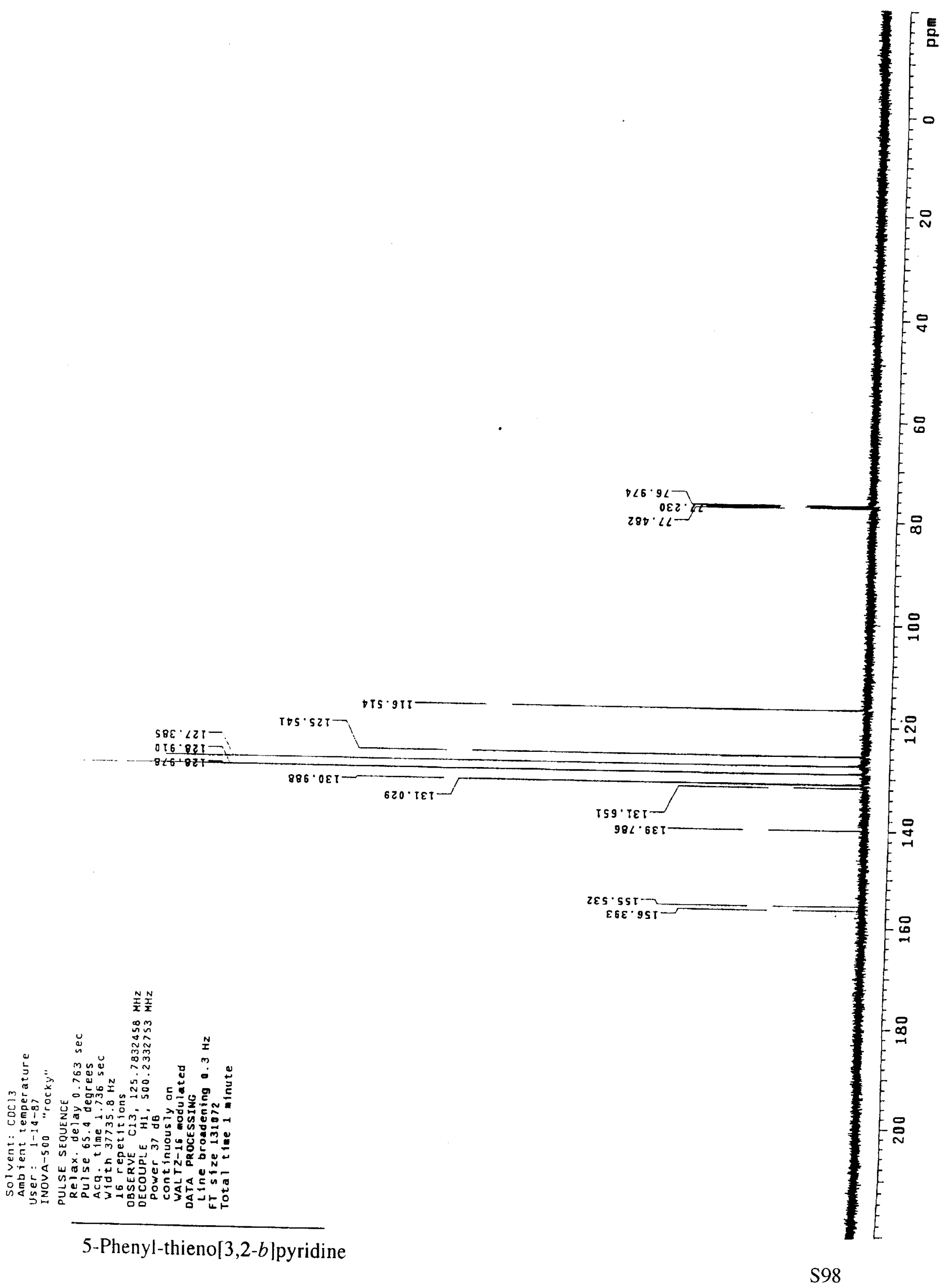




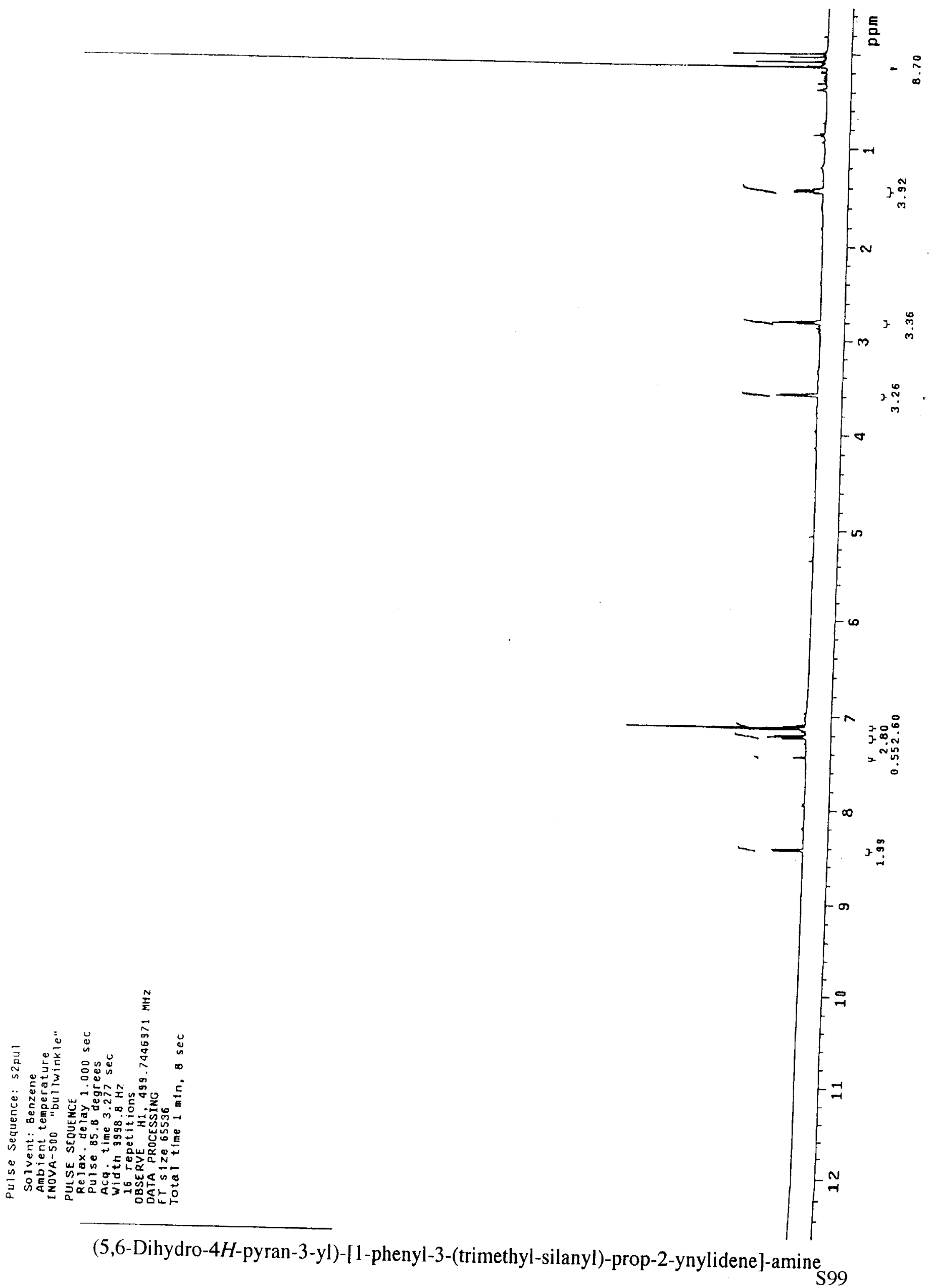




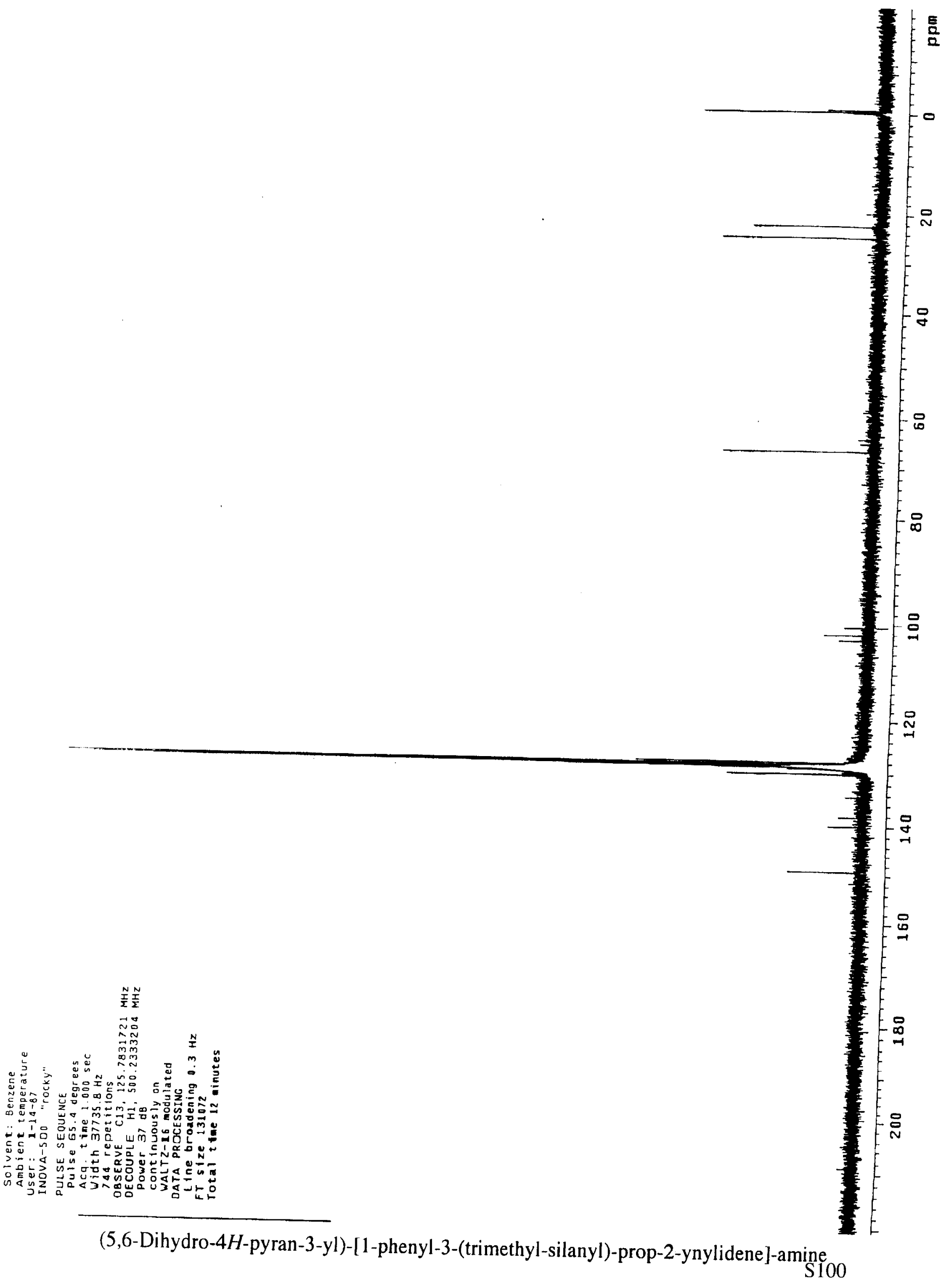




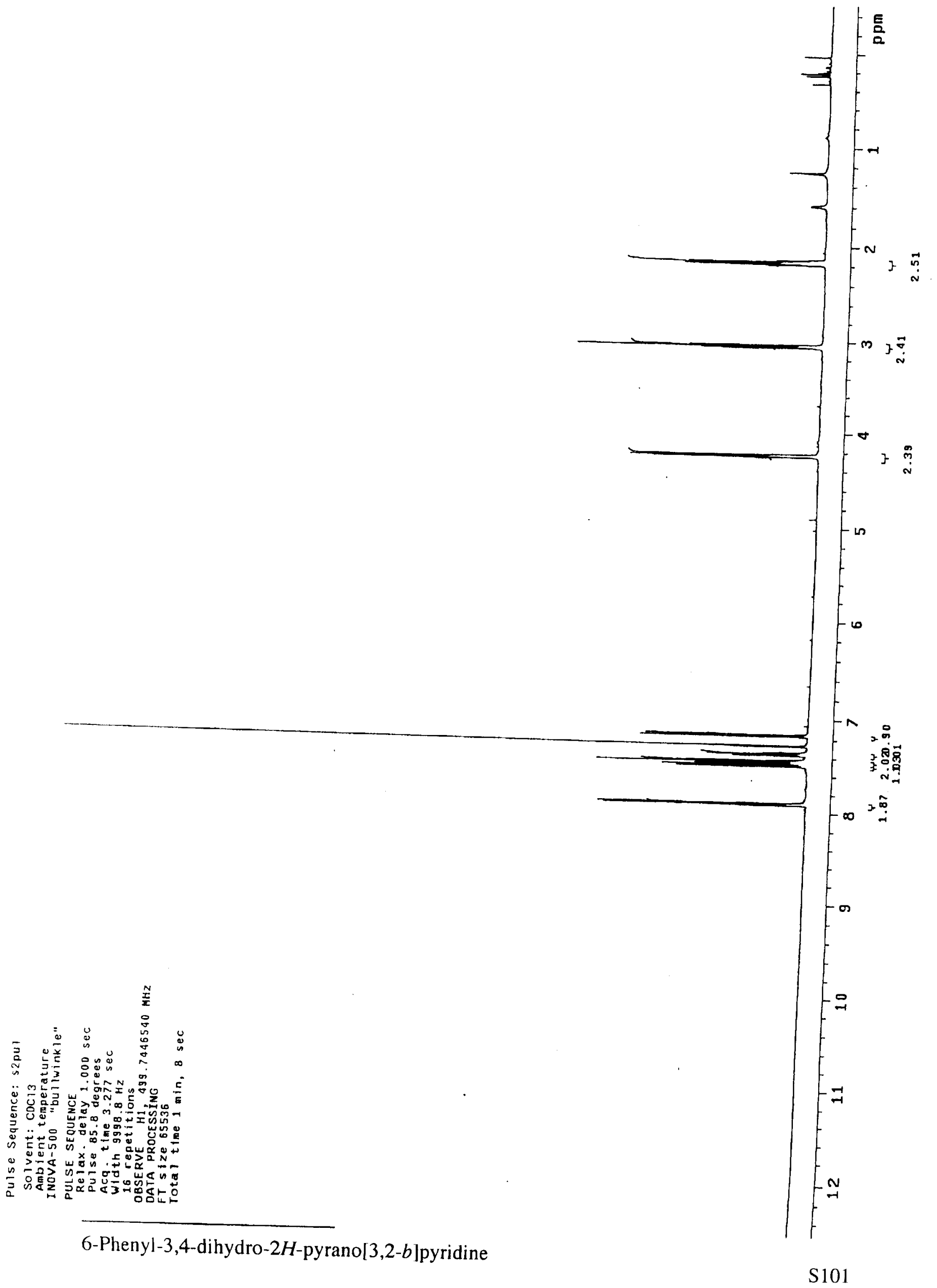




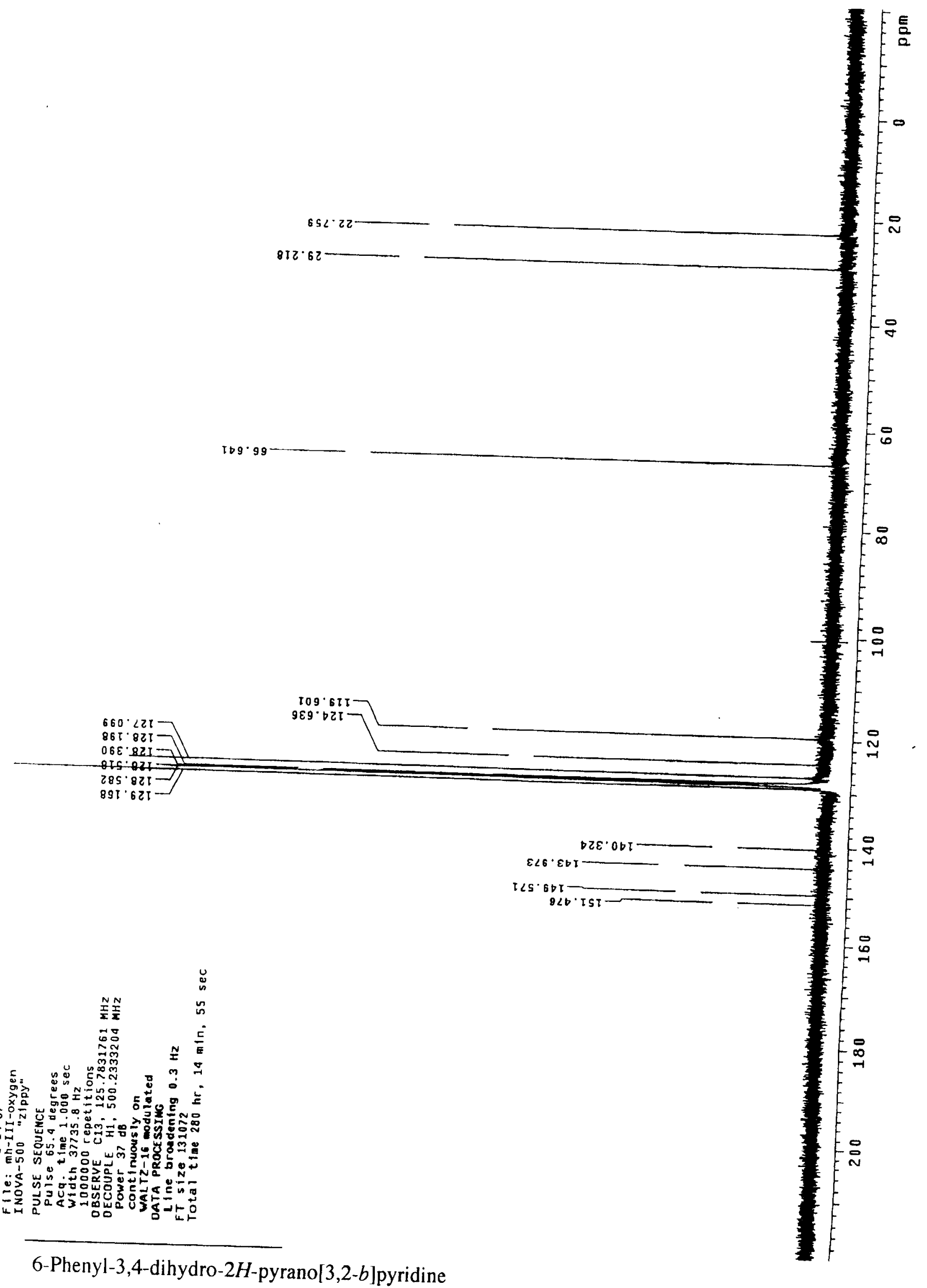




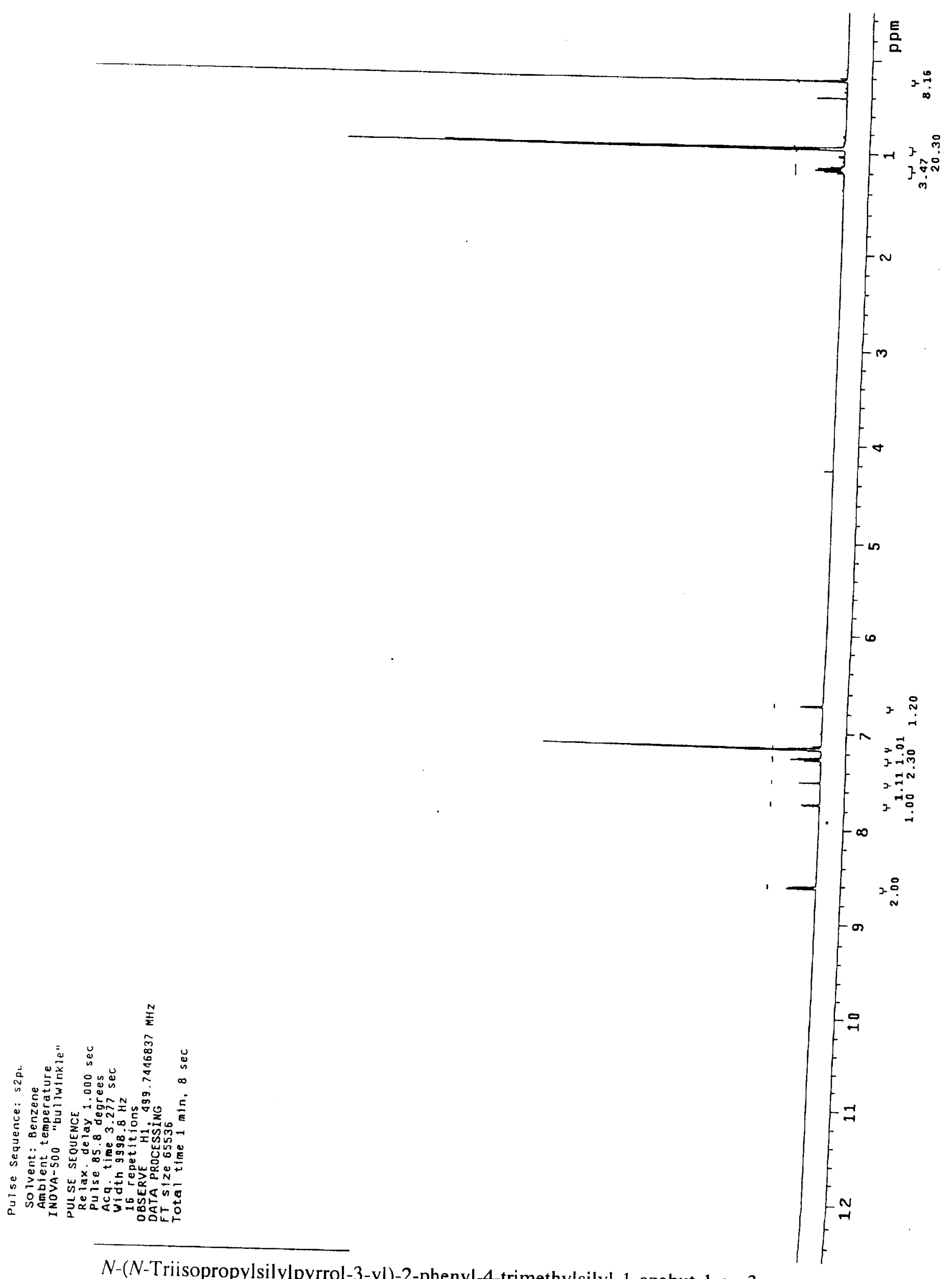

$N$-(N-Triisopropylsilylpyrrol-3-yl)-2-phenyl-4-trimethylsilyl-1-azabut-1-en-3-yne 


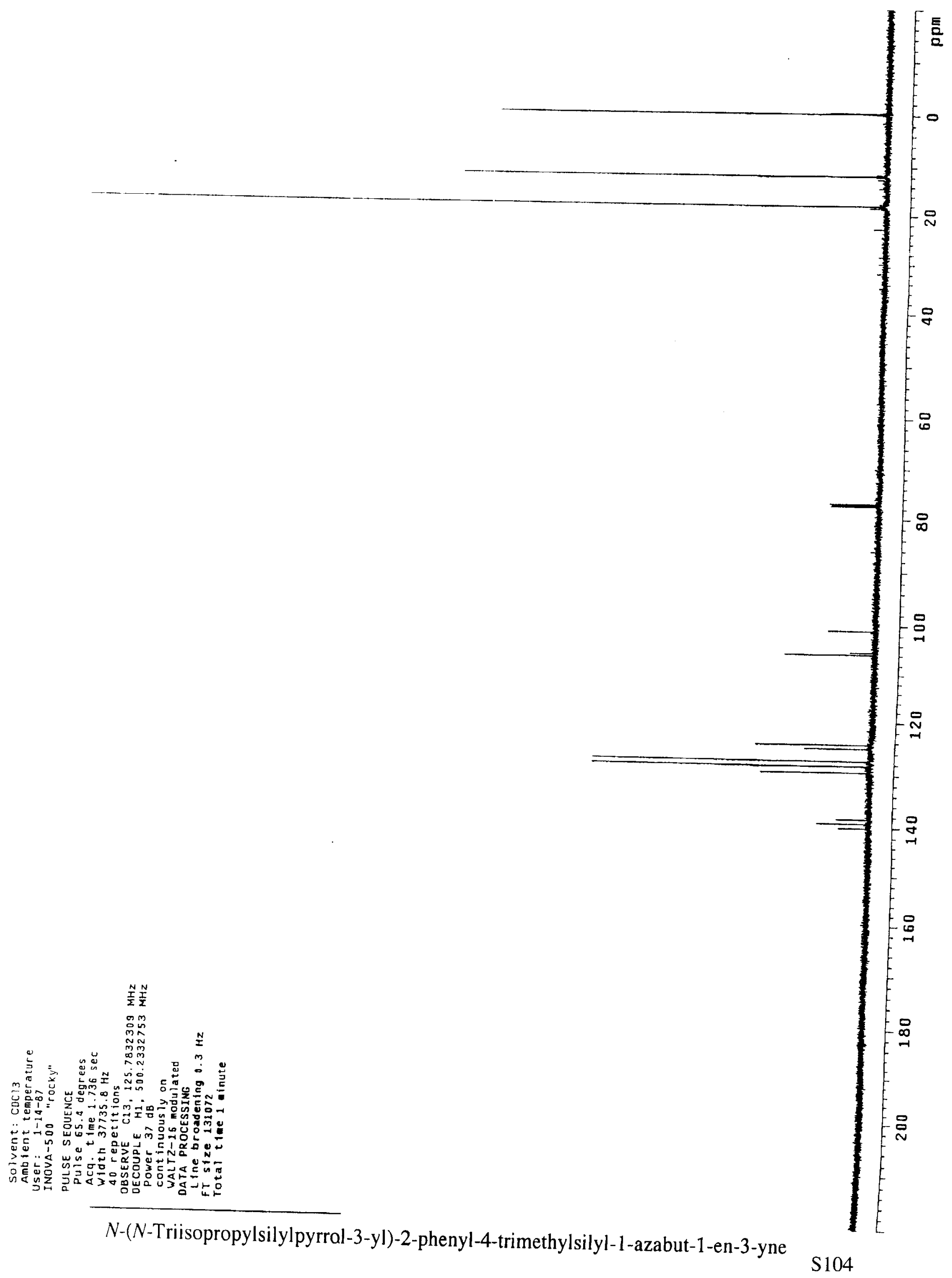




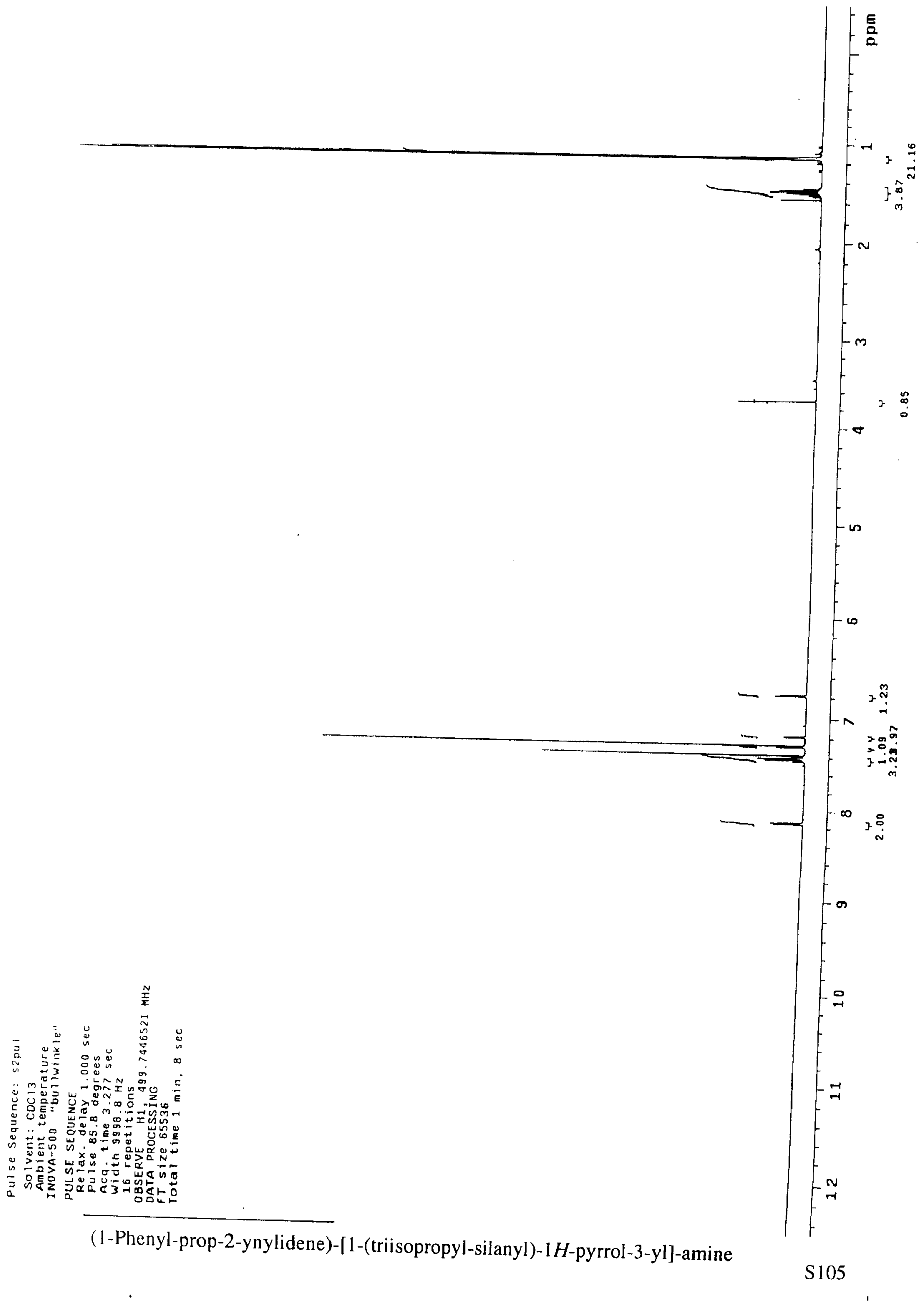




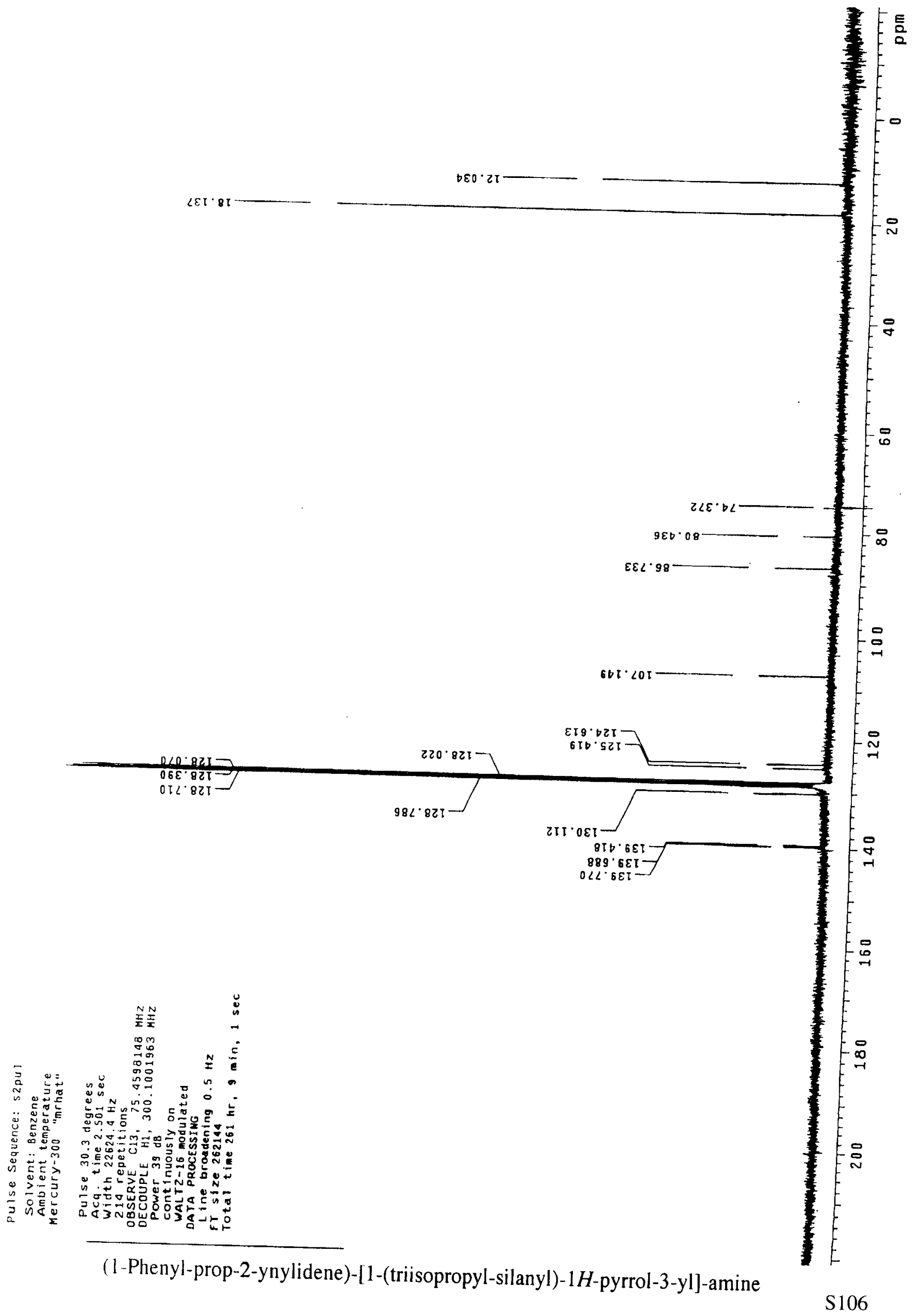




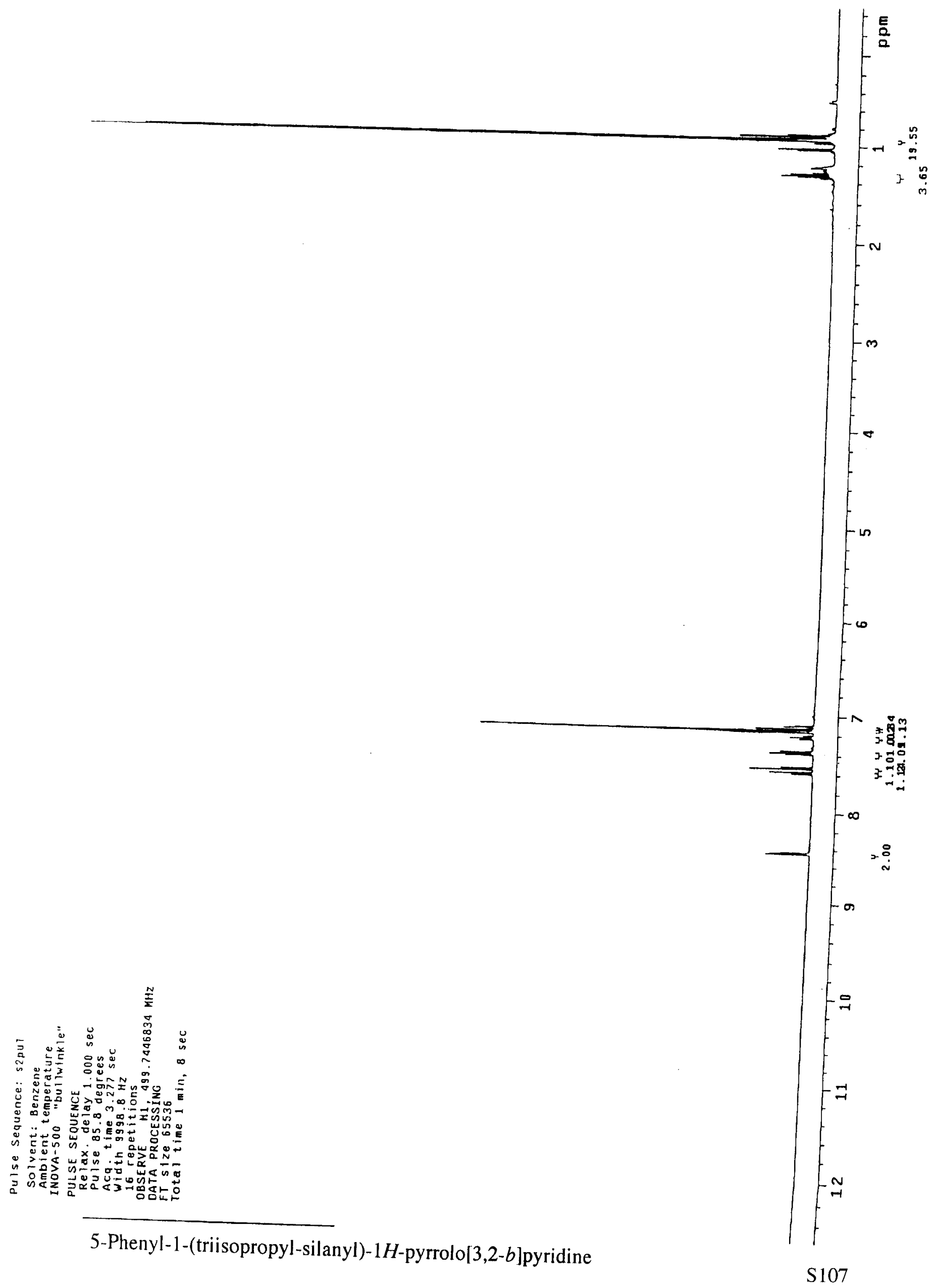




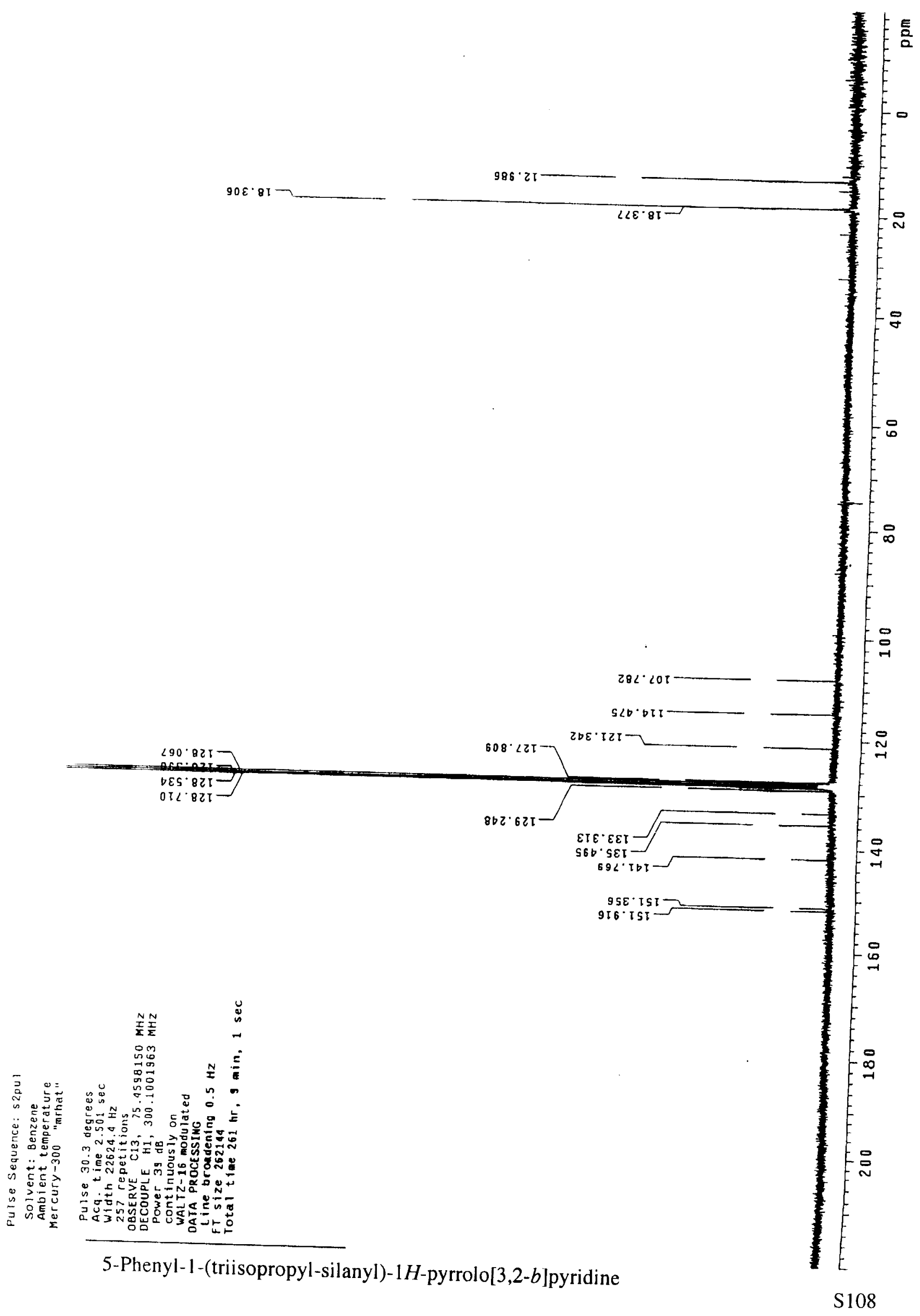




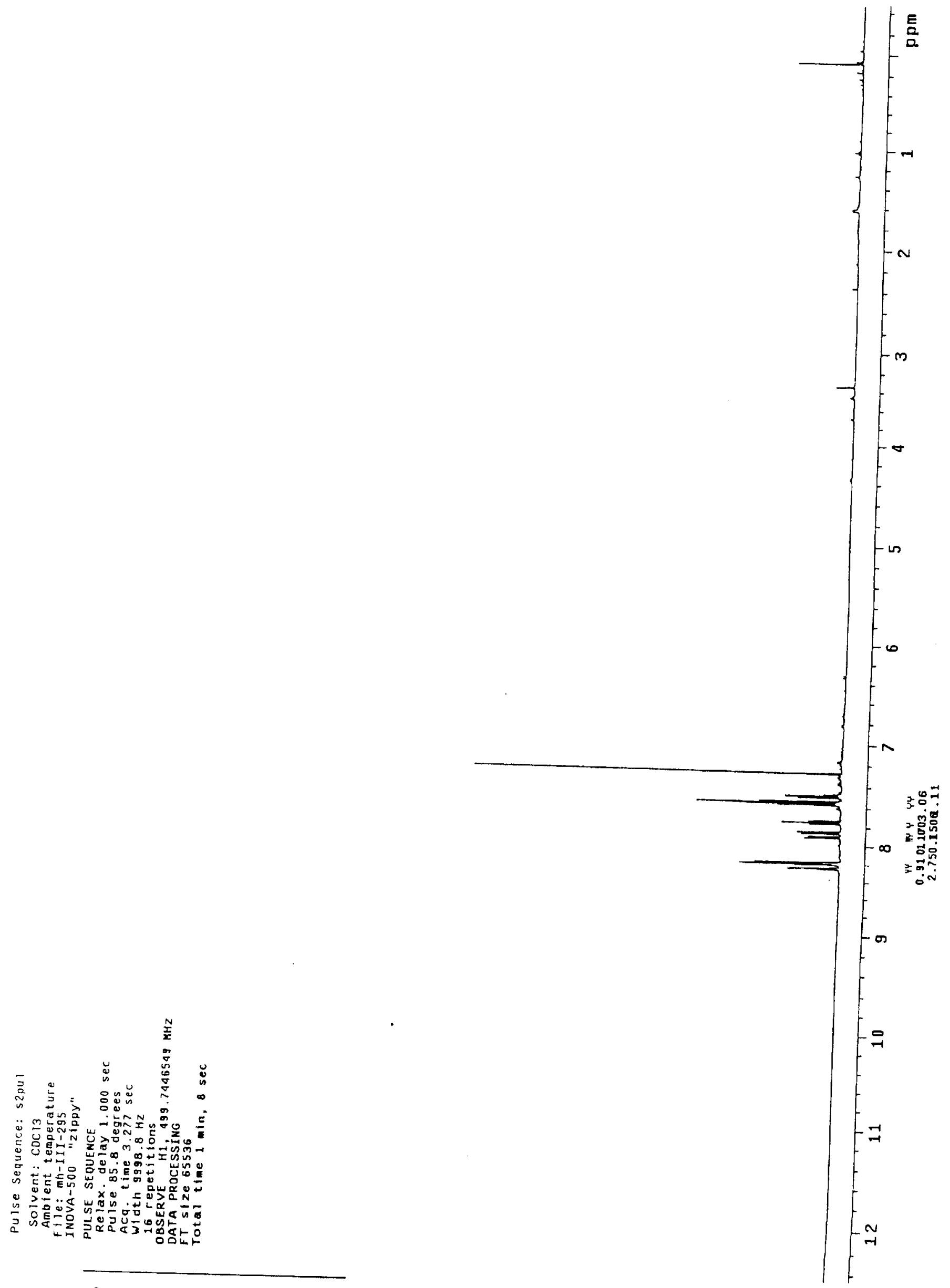

3-Deutero-2-phenylquinoline 


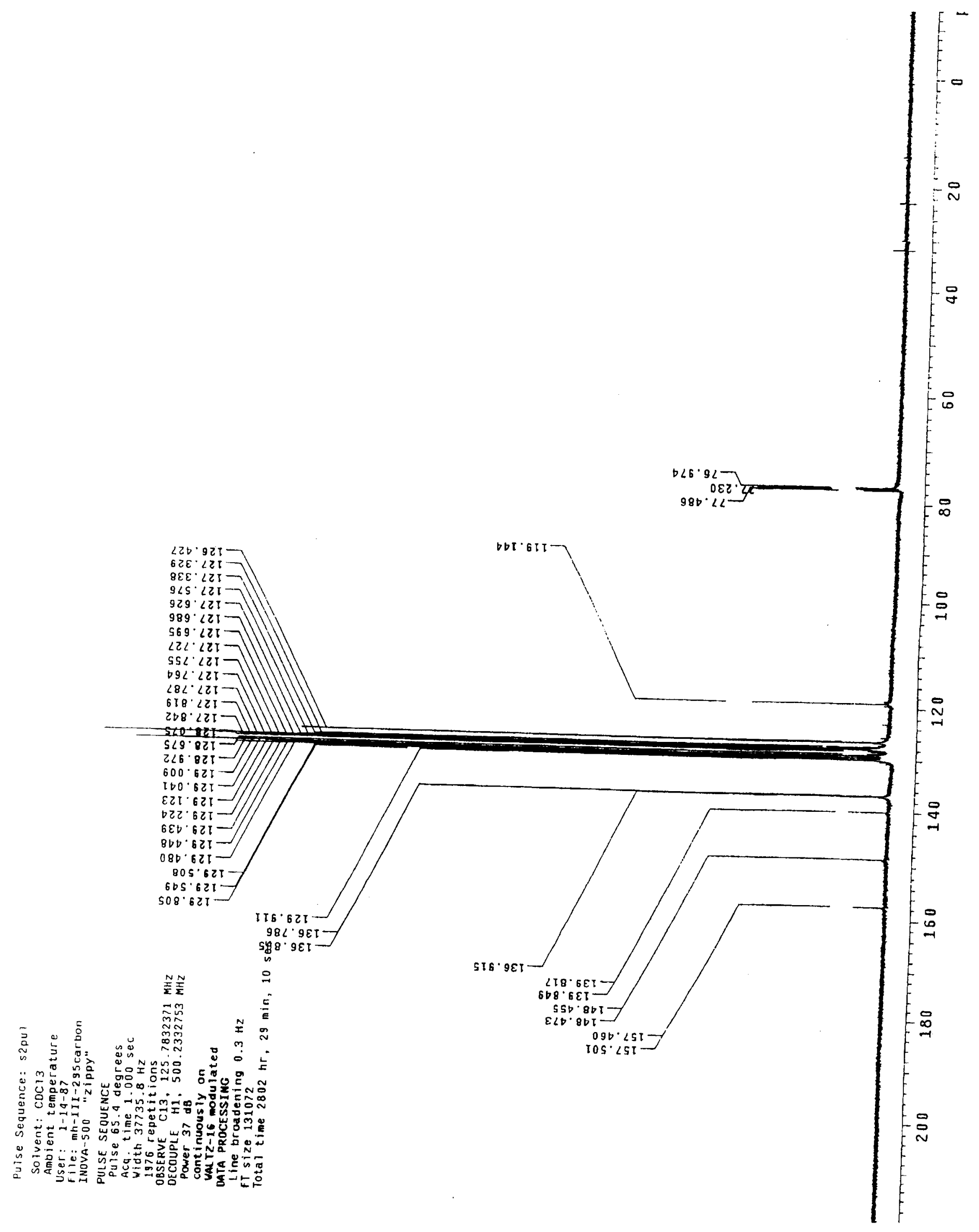

3-Deutero-2-phenylquinoline 


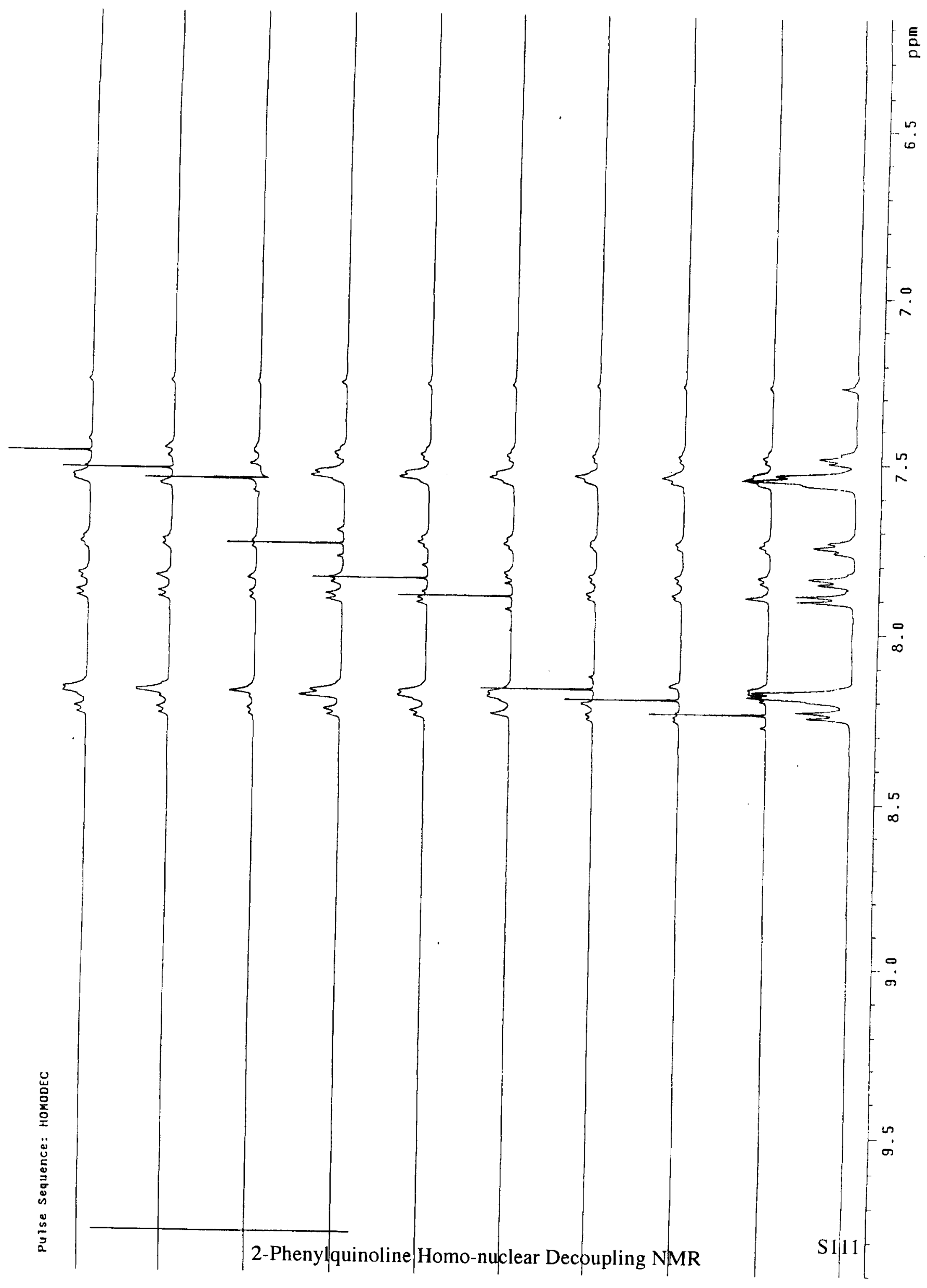



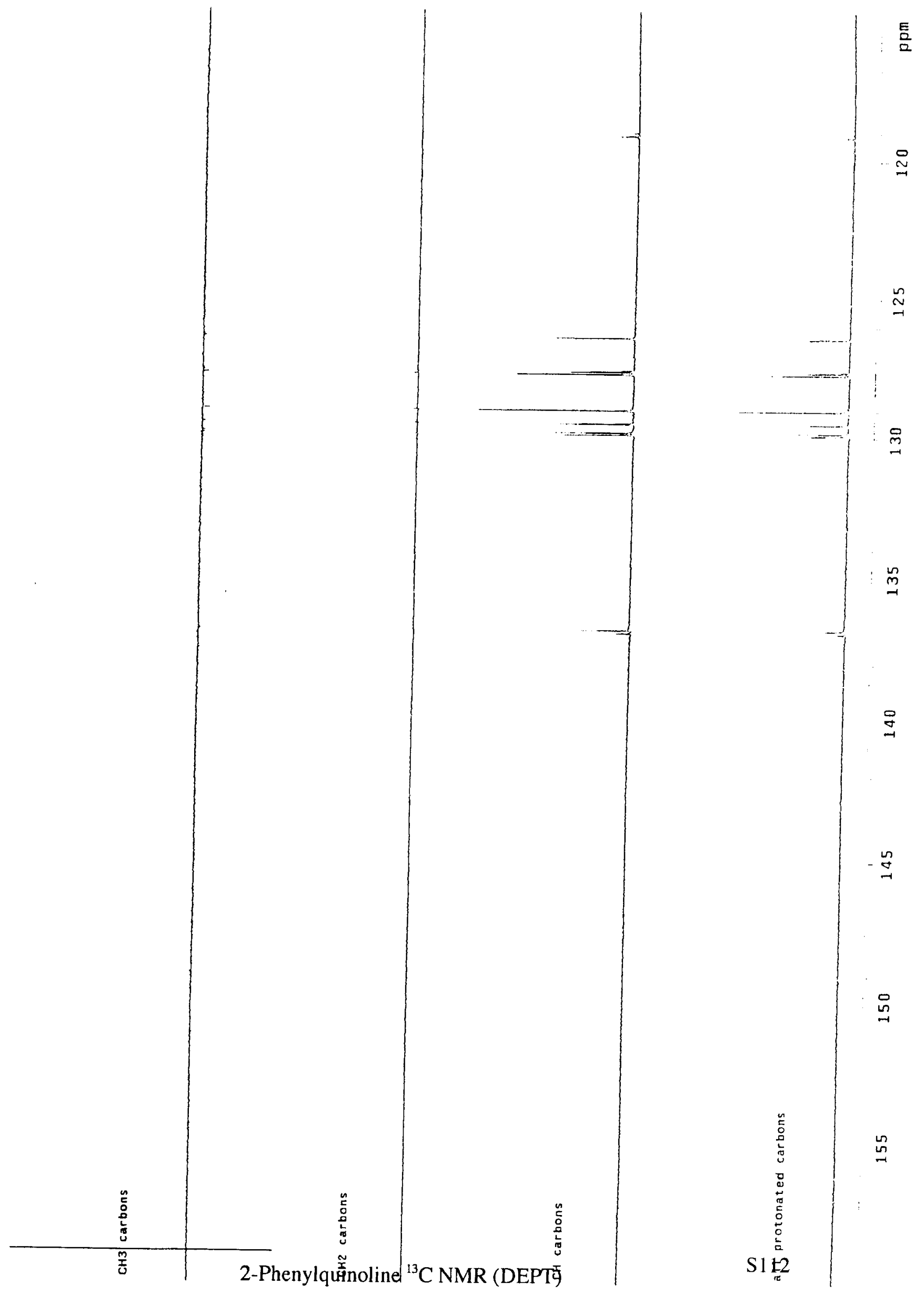


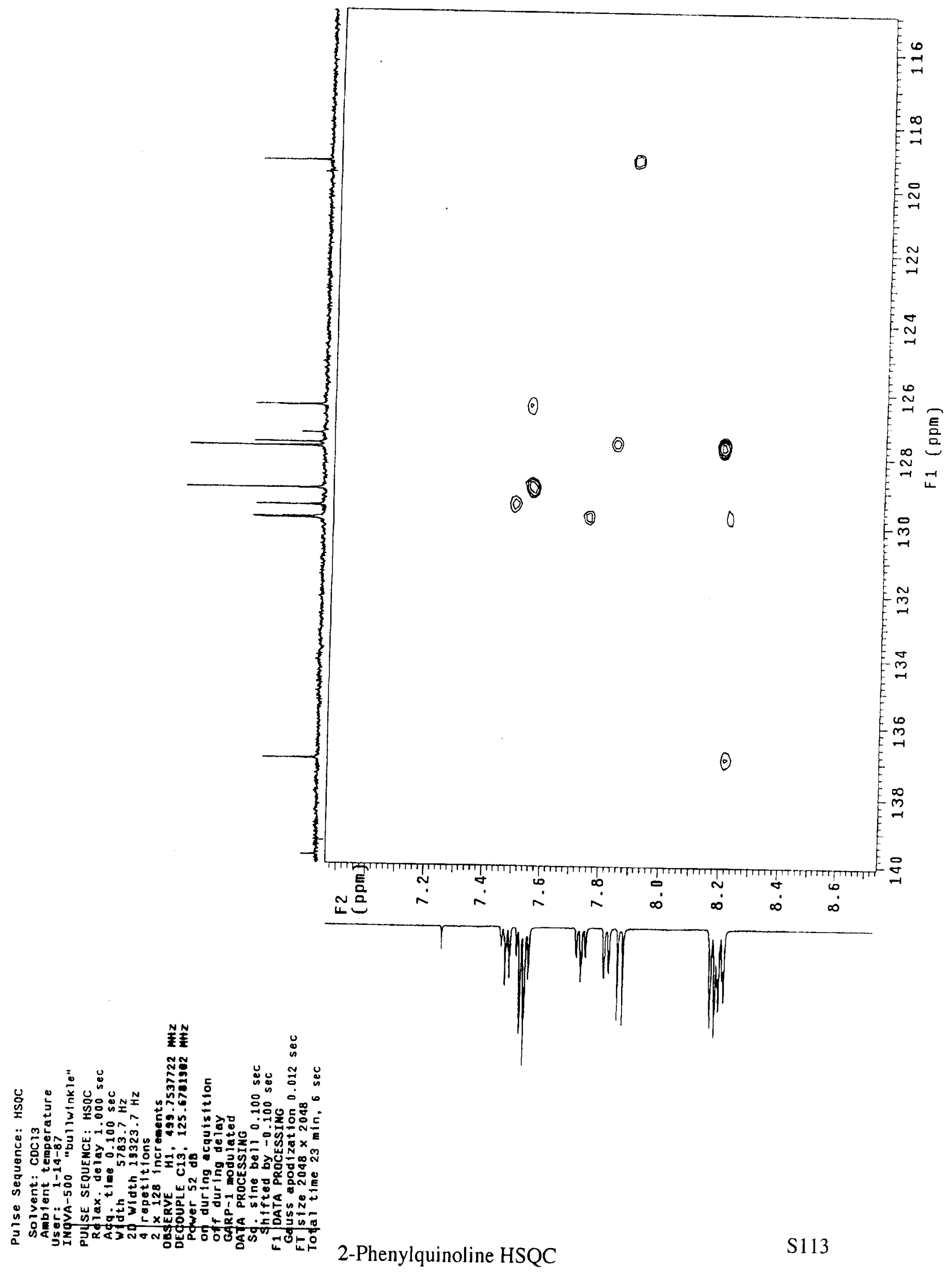

